\title{
An Empirical Ultraviolet Template for Iron Emission in Quasars as Derived from I Zwicky 1
}

\section{Citation}

Vestergaard, M., and B. J. Wilkes. 2001. "An Empirical Ultraviolet Template for Iron Emission in Quasars as Derived from I Zwicky 1." The Astrophysical Journal Supplement Series 134 (1) (May): 1-33. doi:10.1086/320357.

\section{Published Version}

doi:10.1086/320357

\section{Permanent link}

http://nrs.harvard.edu/urn-3:HUL.InstRepos:30212159

\section{Terms of Use}

This article was downloaded from Harvard University's DASH repository, and is made available under the terms and conditions applicable to Other Posted Material, as set forth at http:// nrs.harvard.edu/urn-3:HUL.InstRepos:dash.current.terms-of-use\#LAA

\section{Share Your Story}

The Harvard community has made this article openly available.

Please share how this access benefits you. Submit a story.

\section{Accessibility}




\title{
AN EMPIRICAL ULTRAVIOLET TEMPLATE FOR IRON EMISSION IN QUASARS AS DERIVED FROM I ZWICKY $1^{1}$
}

\author{
M. VeSTERGAARD ${ }^{2,3,4}$ AND B. J. WiLKes ${ }^{2}$ \\ Received 1998 February 16; accepted 2000 December 12
}

\begin{abstract}
We present an empirical template spectrum suitable for fitting and subtracting/studying the $\mathrm{Fe}$ II and Fe III emission lines in the rest frame ultraviolet spectra of quasars and active galactic nuclei, the first empirical ultraviolet iron template to cover the full range of 1250-3090 A. Iron emission is often a severe contaminant in optical-ultraviolet spectra of active galactic nuclei and quasars. Its presence complicates and limits the accuracy of measurements of both strong and weak emission lines and the continuum emission, affecting studies of line and continuum interrelations, the ionization structure, and elemental abundances in active galaxies and quasars. Despite the wealth of work on modeling the quasar $\mathrm{Fe}$ II emission and the need to account for this emission in observed quasar spectra, there is no ultraviolet template electronically available to aid this process. The iron template we present is based on Hubble Space Telescope spectra of the narrow line Seyfert 1 galaxy, I Zwicky 1 (I Zw 1, $z=0.061$ ). The intrinsic narrow lines $\left(\gtrsim 900 \mathrm{~km} \mathrm{~s}^{-1}\right)$ of this source and its rich iron spectrum make the template particularly suitable for use with most active galactic nuclei and quasar spectra. The iron emission spectrum, the line identifications, and the measurements of absorption and emission lines are presented and compared with the work of Laor et al. Comments on each individual line feature and the line fitting are available in Appendix A. The methods used to develop and apply the template are also described. We illustrate the application of the derived $\mathrm{Fe}$ II and $\mathrm{Fe}$ III templates by fitting and subtracting iron emission from the spectra of four high-redshift quasars and of the nearby quasar, 3C 273, confirming their general applicability to active galaxies despite the somewhat unusual properties of I $\mathrm{Zw} 1$. We briefly discuss the small discrepancies between the observed iron emission of these quasars and the ultraviolet template, and compare the template with previously published ones. We discuss the advantages and limitations of the UV Fe II and $\mathrm{Fe}$ III templates and of the template fitting method. We conclude that the templates work sufficiently well to be a valuable and important tool for eliminating and studying the iron emission in active galaxies, at least until accurate theoretical iron emission models are developed. The Si IV $+\mathrm{O}$ IV] $\lambda 1400$ feature in I Zw 1 is clearly strong relative to C IV $\lambda 1549$, and C IV and C III] $\lambda 1909$ are both relatively weak. This may partially be due to the higher densities and lower ionization parameter prevailing in narrow line Seyfert 1 galaxies and to the big blue bump shifting toward lower energies in more luminous Seyferts, such as I $\mathrm{Zw} 1$. In I $\mathrm{Zw} 1$ the narrow line width reveals that $\mathrm{C}$ III] is heavily blended with Si III] $\lambda 1892$, Al III $\lambda \lambda 1854,1863$, and Fe III transitions. This suggests that the $\mathrm{C}$ III] line strength and width may be overestimated in many quasar line studies where the lines are broader and deblending is not possible. This affects density estimates of the broad line region. Photoionization modeling, including all these line features, and subsequent fitting to the spectra are required to estimate the true $\mathrm{C} \mathrm{III]}$ strength. We also argue, based on earlier work, that (strong) iron emission may be connected with high densities and associated with outflows.
\end{abstract}

Subject headings: galaxies: active - galaxies: individual (I $\mathrm{Zw} 1)$ - galaxies: Seyfert methods: data analysis - quasars: emission lines

On-line material: machine-readable tables

\footnotetext{
${ }^{1}$ Based on observations made with the NASA/ESA Hubble Space Telescope, obtained from the data archive at the Space Telescope Science Institute. STScI is operated by the Association of Universities for Research in Astronomy, Inc., under the NASA contract NAS 5-26555.

${ }^{2}$ Harvard-Smithsonian Center for Astrophysics, 60 Garden Street, Cambridge, MA 02138.

${ }^{3}$ The Niels Bohr Institute for Astronomy, Physics and Geophysics, Copenhagen University Observatory, Juliane Maries Vej 30, DK-2100 Copenhagen Ø, Denmark.

${ }^{4}$ Current address: Department of Astronomy, The Ohio State University, 140 West 18th Avenue, Columbus, OH 43210-1173; vester@astronomy.ohio-state.edu.
}

\section{INTRODUCTION}

Quasar $^{5}$ ultraviolet (UV) spectra characteristically contain broad emission lines originating in the central ( light year) region, the broad line region (BLR). The strongest lines are (the resonance lines) $\mathrm{Ly} \alpha$ 21216, Si IV + O IV] $\lambda 1400, C$ IV $\lambda \lambda 1548,1551, C$ III] $\lambda 1909$, and

\footnotetext{
5 "Quasars" here refers to both the radio-loud and radio-quiet subgroups.
} 
Mg II $\lambda \lambda 2796,2803$. For a number of years after the discovery of quasars these lines and a few iron transitions (Greenstein \& Schmidt 1964; Wampler \& Oke 1967) were the only lines detected in their UV spectra. With the availability of high signal-to-noise ratio $(\mathrm{S} / \mathrm{N})$, high-resolution spectra, and in particular data taken with Hopkins Ultraviolet Telescope (e.g., Kriss et al. 1992; Zheng et al. 1995; Zheng, Kriss, \& Davidsen 1996), Hubble Space Telescope (HST) (e.g., Laor et al. 1994, 1995; Zheng et al. 1997; Kriss et al. 2000; Kraemer \& Crenshaw 2000), and the Keck telescope (e.g., Tran, Cohen, \& Goodrich 1995; Brotherton et al. 1997; Barlow \& Sargent 1997; Larkin et al. 2000; Carson et al. 2000), it has become obvious that quasar spectra contain a plethora of weak lines as well.

\subsection{The Need for Fitting and Removal of Iron Emission}

In order to study e.g., the relationship between the continuum and the line emission, the dynamical structure, the ionization balance and structure, or the chemical abundances in active galactic nuclei (AGNs) and quasars it is important to make reliable measurements of the continuum emission, the strong broad lines including the wings of their profiles, and the weak emission lines. In addition to hydrogen, helium and the above-mentioned elements, AGNs and quasars also contain atomic and ionic iron, the stable end product of nucleosynthesis. Due to the large number of electron levels in iron atoms, thousands of emission line transitions are distributed throughout the UV and optical spectral regions. The weak lines, iron as well as those not being iron transitions (hereafter "non-iron" lines), blend together, partly due to the associated transitions being very close or overlapping in energy, and partly due to the high (presumed) dynamic velocities of the broad-line emitting clouds broadening the lines. Line widths (FWHM, the full width at half-peak flux) of up to $10000 \mathrm{~km} \mathrm{~s}^{-1}$ have been measured (Wilkes 2000). This heavy blending of weaker lines, dominated by iron transitions, forms a pseudocontinuum above the intrinsically emitted continuum (see, e.g., Figs. 6 and 7) even in high $\mathrm{S} / \mathrm{N}$ quasar spectra (Wills et al. 1985; Boroson \& Green 1992, hereafter BG92; Wills et al. 1995). This pseudo-continuum severely complicates the study of both weak non-iron features, particularly important for abundance and ionization studies, and the wings of strong resonance lines. The uncertainty introduced into broad emission-line measurements by iron emission "contamination" can be relatively large: excluding very weak lines where it can reach $\sim 100 \%$, a rough estimate is $5 \%-50 \%$ based on Wilkes (1984) and our own line measurements before and after iron emission removal using the template presented here $(\S 4)$. The exact level of uncertainty depends on the line transition, the line parameter measured and the overall strength of the emission line spectrum. Moreover, the relative strengths of the iron features vary greatly from object to object. These uncertainties are dominated by the difficulty in determining an accurate continuum level, one of the main problems in quasar line studies, although such errors are seldom quoted. Combined, these uncertainties provide a strong argument that all quasar spectral studies should include iron emission fitting and removal.

These limitations imposed by the contaminating iron emission have been known for some time (e.g., Wills \& Browne 1986). A possible solution to the continuum level uncertainty is to use only wavelength ranges which contain pure continuum emission (so-called continuum windows; Francis et al. 1991) when fitting the continuum. However, few such regions exist and they are generally small, especially in the UV spectral region, 1000-2000 A. Moreover, in objects with very broad lines even these line-free regions may be contaminated by broad line-wing emission (Boroson, Persson, \& Oke 1985; Wills \& Browne 1986).

\subsection{Earlier Work on Iron Emission}

Allowance for the optical and UV iron emission has not extensively been made, in spite of the increasing recognition of its importance for broad line studies. It was rendered a very hard task in the 1980's by the lack of sufficient atomic data to allow identification of the iron transitions and high spectral resolution to resolve them. Earlier work includes identifying the UV iron emission lines in AGNs [e.g., Wills, Netzer, \& Wills 1980; Penston 1980; Penston et al. 1983 (in stars); Hartig \& Baldwin 1986; Johansson \& Jordan 1984] and modeling this emission (e.g., Netzer 1980; Kwan \& Krolik 1981; Netzer \& Wills 1983; Wills et al. 1985; CollinSouffrin et al. 1986; Penston 1987; Collin-Souffrin, Hameury, \& Joly 1988; Krolik \& Kallman 1988; Ferland \& Persson 1989; Dumont \& Collin-Souffrin 1990; Netzer 1990, and references therein). Despite extensive efforts the current theoretical models of the optical and UV iron emission cannot fully reproduce and explain the observations. Fortunately, projects are under way to compute detailed radiative transition probabilities of iron atoms and ions (the IRON Project: Hummer et al. 1993; Nahar, Bautista, \& Pradhan 1997; Nahar et al. 2000, and references therein) and compute photoionization models of AGNs which also take the iron emission into account with or without exact radiative transfer (Sigut \& Pradhan 1998; “The Kentucky group": Verner et al. 1999, and references therein) based on improved atomic data (e.g., the Opacity Project: Seaton et al. 1994). Other studies presenting iron line lists and radiative transition probabilities, which are also used here, include Fuhr, Martin, \& Wiese (1988), Ekberg (1993), Giridhar \& Arellano Ferro (1995), Nahar (1995), Nahar \& Pradhan (1996), Quinet (1996), Quinet, Le Dourneuf, \& Zeippen (1996), and Kurucz (1997). These iron line lists are available on the World Wide Web.

\subsection{Iron Emission Correction}

Phillips (1977) was one of the first to compare the spectrum of I Zw 1 to other Seyfert galaxy spectra by broadening the former with a Gaussian profile, selected to match the line widths in the latter. Phillips did not subtract the iron emission but confirmed his suspicion that the $\mathrm{Fe}$ II and $\mathrm{H}$ I lines have essentially the same widths and profiles. Wills et al. (1985) simulated the Fe II emission and absorption in eight low to intermediate redshift quasars using photoionization modeling of $\sim 3000$ iron lines. In spite of the limitations imposed by the atomic data and computing speed at the time, they were able to reproduce the UV-optical spectra reasonably well. Some deviations from the observed spectra are, however, seen (see $\S 3.4$ ). The photoionization models presented by Wills et al. are specific to their individual object spectra and were not generated with a general subtraction of iron emission in AGNs in mind, where a typical iron spectrum is more appropriate. Boroson, Persson, \& Oke (1985) made one of the first attempts to take the effects of the iron emission on broad-line studies into direct account. They estimated the uncertainty associ- 
ated with line (equivalent width, EW) measurements as a function of line widths by broadening an AGN spectrum by a Hanning profile (triangular) with a range of widths. They found the measured EWs to weaken with increasing FWHM because the continuum level becomes systematically overestimated, as the iron lines broaden and blend to form a pseudo-continuum. As a consequence, narrow lines incorrectly appear stronger. This may explain why quasars, with their broad emission lines were not recognized early on to be strong iron emitters ${ }^{6}$ (Phillips 1977; Davidson \& Netzer 1979). One way of correcting for the iron emission is to model it using the profile information of the $\mathrm{H} \beta$ line, as the isolated $\mathrm{Fe}$ II and $\mathrm{H} \beta$ profiles are observed to have both similar widths and profile shapes (Phillips 1977; Laor et al. 1997b, hereafter L97). Thus, a synthetic spectrum of the iron emission can be constructed by shifting and scaling $\mathrm{H} \beta$ profile templates according to a list of iron line positions and relative strengths. However, this method depends highly on the atomic data lists to be representative, in terms of accuracy and completeness, of the iron emission in AGNs and quasars. As mentioned, theoretical studies are not yet able to fully reproduce the observed iron emission, but work is in progress (D. Verner 1998, private communication; Verner et al. 1999; see also § 3.4).

A significant improvement in the accuracy of broad emission line measurements can be obtained by using an observed $\mathrm{AGN}^{7}$ or quasar iron emission template to fit and subtract the iron emission in quasar spectra before performing line and continuum measurements. The benefits of using such a template, containing all the iron transitions typically present in quasars, are manifold. Combined with the much improved atomic data, becoming increasingly available $(\S 1.2)$, empirical templates provide a powerful tool to study the observed iron spectrum in terms of theoretical models. This template method was adopted by BG92, who successfully used an optical (4250-7000 $)$ iron template based on the nearby, narrow-line Seyfert 1 (NLS1; Osterbrock \& Pogge 1985), I Zw 1, on the Bright Quasar Survey sample. Their method is described in $\S 4.2$. Corbin \& Boroson (1996) use the same method with a $2300-3000 \AA$ UV iron template of I Zw 1 . See $\S 3.4$ for a comparison with this template.

The advent of HST UV quasar spectral data allows us to extend the template method into the UV regime. We here present, to our knowledge, the first high $\mathrm{S} / \mathrm{N}$, highresolution, quasar empirical UV iron template spectrum ranging from rest frame 1250 to $3090 \AA$ which is applicable to quasar data. The template is based on HST (archival) data of I Zw 1 (L97). The method with which the template was generated is an extension of that of BG92 and Corbin \& Boroson (1996) including a more detailed fitting of the lines which are not iron (see $\S 3$ for details and $\S 3.4$ for a

\footnotetext{
${ }^{6}$ The fact that radio-loud quasars were often studied more than radioquiets, due to their easily detectable, strong radio emission, may also have contributed, because radio-quiet quasars show relatively stronger optical iron emission (Peterson, Foltz, \& Byard 1981; Bergeron \& Kunth 1984; Corbin 1997).

${ }^{7}$ The formal distinction between the use of the term "AGN" and "quasar" is only that of the object's luminosity, with quasars occupying the most powerful end of the luminosity range of active galactic nuclei $\left(M_{V}<-23\right.$, Véron-Cetty \& Véron 1993). As the methods and iron templates described here are applicable to AGNs as well, this fact is silently assumed in the following, when we refer to quasars only, in addressing the use of the template.
}

discussion). When optical data between 3000 and $4250 \AA$ become available (L97 present 3000-3800 ̊ data), suitable for production of an intermediate wavelength iron template, the available templates will provide a powerful tool for consistent modeling of the UV and optical iron emission throughout the 1250-7000 ̊̊ region of quasar spectra.

The procedure of fitting the iron emission using an empirical template assumes that (1) the iron spectrum of $\mathrm{I} Z \mathrm{~W} 1$ is representative of quasar iron emission spectra and that (2) all quasars have similar iron spectra to within a scaling factor and/or a line profile broadening. Not enough is currently known about the iron emission in AGNs to firmly assess the validity of these assumptions. Though until theoretical models can explain the observed iron emission more confidently, this template fitting procedure is the best available approach. We test the method by fitting several representative quasar spectra $(\S 4.3)$ showing that the iron emission can be well fitted if allowed the freedom to vary some of the multiplet ratios in the empirical template, indicating that the basic assumptions are valid.

The iron emission templates have importance not only for our ability to fit and subtract the iron emission in quasar spectra, but also as tools with which we can study the iron emission strengths themselves. Iron is a key coolant emitting $\sim 25 \%$ of the total energy output from the BLR (Wills et al. 1985; Boller, Brandt, \& Fink 1996), emphasizing the importance of including the iron emission in studies of the BLR.

\subsection{The Narrow-Line Seyfert $1, I Z w 1$}

The spectra of NLS1 galaxies are particularly useful for generating empirical iron templates because their strong and rich iron emission allows detection of weak iron features and identification of as many iron transitions (Figs. 2 and 4, Table $4, \S 3.3 .1$ ) as are typically present in AGN spectra. The relatively narrow width (FWHM $\lesssim 2000 \mathrm{~km}$ $\mathrm{s}^{-1}$ ) of the broad emission lines permits the individual noniron lines to be resolved, isolated, and removed from the spectrum. It also allows us to match the line width in most iron-contaminated AGN spectra so as to fit and subtract the iron emission by broadening the iron template.

I Zw 1 (PG 0050+124; $z=0.061$, see $\S 2$ ) is classified as a NLS1 and has the "narrow" broad emission lines, strong Fe II emission (e.g., Phillips 1976; Osterbrock \& Pogge 1985; Lipari, Terlevich, \& Macchetto 1993; Pogge 2000; Rodríguez-Ardila, Pastoriza, \& Donzelli 2000; Rudy et al. 2000; but see also Gaskell 2000), steep soft X-ray spectra (Boller et al. 1996), and strong far-IR emission (Halpern \& Oke 1987), typical of this class. Their radio properties, however, are similar to those of other Seyfert galaxies (Ulvestad, Antonucci, \& Goodrich 1995). They are also variable at optical and UV wavelengths (e.g., Zwicky 1971; Giannuzzo \& Stirpe 1996; Rodríguez-Pascual, Mas-Hesse, \& Santos-Lleo 1997; Leighly 1999; Miller et al. 2000) and the fastest X-ray variable AGNs known (see discussion by Boller et al. 1996). I Zw 1 is a well-studied object thanks in part to its relative brightness (Schmidt \& Green 1983), its exceptionally narrow lines, its iron-rich spectrum (Sargent 1968; Phillips 1976, 1977; Oke \& Lauer 1979; Boroson et al. 1985; Halpern \& Oke 1987; BG92; L97), and its richness in low-ionization lines (Persson \& McGregor 1985; van Groningen 1993; L97; Table 3). I Zw 1 is also an infrared (IR) luminous source (Rieke \& Low 1972; Rieke 1978; Halpern \& Oke 1987) and has been observed in $\mathrm{CO}$ and $\mathrm{H}_{2}$ 
TABLE 1

LOG OF OBSERVATIONS OF I Zw 1 (PG 0050+124)

\begin{tabular}{ccccccccc}
\hline \hline Grating & Date (UT) & $\begin{array}{c}\lambda \text { Range } \\
(\AA)\end{array}$ & $\begin{array}{c}\Delta \lambda \\
\left(\AA \text { pixel }^{-1}\right)\end{array}$ & $\begin{array}{c}\text { Res. }^{\mathrm{a}} \\
(\AA)\end{array}$ & Detector & $\begin{array}{c}\text { Aperture } \\
(\operatorname{arcsec})\end{array}$ & $\begin{array}{c}\text { Exposure Time } \\
(\mathrm{s})\end{array}$ & $\begin{array}{c}\lambda \text { Offsets }^{\mathrm{b}} \\
(\AA)\end{array}$ \\
\hline $\mathrm{G} 130 \mathrm{H} \ldots \ldots$ & $02 / 13 / 94$ & $1087-1606$ & 0.251 & 0.96 & Blue & 0.86 & 29700 & 0.36230 \\
$\mathrm{G} 190 \mathrm{H} \ldots \ldots$. & $09 / 14 / 94$ & $1572-2312$ & 0.359 & 1.39 & Amber & 0.86 & 6030 & 1.10670 \\
$\mathrm{G} 270 \mathrm{H} \ldots \ldots$. & $09 / 14 / 94$ & $2222-3277$ & 0.511 & 1.97 & Amber & 0.86 & 2100 & 1.03755 \\
\hline
\end{tabular}

${ }^{\text {a }}$ Spectral resolution.

${ }^{b}$ Wavelength offset determined from ISM absorption lines; as described in $\S 2$.

molecular lines (Barvainis, Alloin, \& Antonucci 1989; Eckart et al. 1994). Some modeling of the observed optical iron emission was performed by Phillips (1978). I Zw 1 was observed by IUE in 1978-1982 (Courvoisier \& Paltani 1992; Lanzetta, Turnshek, \& Sandoval 1993; Paltani \& Courvoisier 1994), and the source is included in a number of IUE studies addressing larger samples of AGNs (e.g., $\mathrm{Wu}$, Boggess, \& Gull 1983; Pian \& Treves 1993; Wang, Zhou, \& Gao 1996; Rodríguez-Pascual et al. 1997). Condon, Hutchings, \& Gower (1985) detected $21 \mathrm{~cm}$ radio emission from I $\mathrm{Zw} 1$ consistent with emission from a late-type host galaxy.

I Zw 1 is a particularly good choice for an empirical $\mathrm{Fe}$ II template as it is so well studied, especially in terms of its optical iron emission and was used by BG92 and Corbin \& Boroson (1996) for their templates.

Additional candidates of narrow-line, iron-rich AGNs suitable for use as iron templates may be found among other NLS1's. Possibilities include Mrk 957 (5C 3.100), Ark 564, 1E 1226.9+1336, E1228+123, Mrk 507 (1748+687), Mrk $42(1151+465)$, and the less extreme Mrk 291 $(1552+193)$, Mrk $493(1557+352)$ and $1244+026$.

The structure of this paper is as follows: $\S 2$ addresses the data processing, $\S 3$ contains a description of the generation of the template and identification of the various spectral lines, in $\$ 3.4$ comparisons are made with other available empirical UV templates and synthetic Fe II models, § 4 describes the application of the template along with initial results, $\S 5$ is dedicated to a discussion of $I \mathrm{Zw} 1$ and some of its spectral features, and $\S 6$ summarizes the main conclusions. Comments on individual line features are deferred to Appendix A.

\section{DATA PROCESSING}

The data presented here consist of HST Faint Object Spectrograph (FOS) archival spectra of I Zw 1 . The journal of observation and instrumental setup is summarized in Table 1 for convenience (see also L97).

The $\mathrm{G} 130 \mathrm{H}$ spectrum was calibrated according to the standard CALFOS procedure with updated (1996 March) calibration files, as the flux calibration status of the archival data is uncertain. ${ }^{8}$ The pipeline calibrations were used for the $\mathrm{G} 190 \mathrm{H}$ and $\mathrm{G} 270 \mathrm{H}$ spectra. Multiple observations obtained with the same grating were combined by weighting with the exposure time to form a single spectrum for each wavelength region. A color excess, $E(B-V)=N_{\mathrm{H}} / 48$ $\times 10^{20} \mathrm{~cm}^{-2}=0.105 \mathrm{mag}$, was determined based on the Galactic hydrogen column density, $N_{\mathrm{H}}=5.05( \pm 0.1)$

\footnotetext{
${ }^{8}$ The data were skipped by the pipeline calibration, evident from the data intensity level and the missing flux calibration flags in the data headers.
}

$\times 10^{20} \mathrm{~cm}^{-2}$, observed ${ }^{9}$ by Stark et al. (1992). The spectra were dereddened using the average extinction curve presented by Cardelli, Clayton, \& Mathis (1989), using $A_{V}=$ $3.1 * E(B-V)$ and the IRAF ${ }^{10}$ (V2.11) task "deredden." Due to the average nature of this curve, some residual dust extinction features may be present in the spectra, especially in the $2200 \AA$ region (Fig. 1), where characteristic dust extinction features are generally expected (e.g., Osterbrock 1989). Iron emission in this region complicates this identification. We corrected for an offset in the wavelength solution, due to the nonzero uncertainty in the wavelength calibration (typical uncertainties are $\sim 0.25 \AA$ in $\mathrm{G} 130 \mathrm{H}$, $\sim 0.37 \AA$ in $\mathrm{G} 190 \mathrm{H}, \sim 0.52 \AA$ in $\mathrm{G} 270 \mathrm{H}$; Leitherer 1995), by comparing the observed wavelengths of the galactic interstellar medium (ISM) absorption lines with their laboratory wavelengths. The absorption lines used are $\mathrm{Si}$ II $\lambda 1190, \mathrm{Si}$ II $\lambda 1193$, Si III $\lambda 1206$, Si II $\lambda 1260, \mathrm{C}$ II $\lambda 1335, \mathrm{Si}$ II $\lambda 1527, \mathrm{Al}$ II $\lambda 1671, \mathrm{Fe}$ II $\lambda 2344, \mathrm{Fe}$ II $\lambda 2374, \mathrm{Fe}$ II $\lambda 2382, \mathrm{Fe}$ II $\lambda 2586, \mathrm{Fe}$ II 22600, Mg II $\lambda 22796,2803$, and Mg I 22853 (Table 2). The applied wavelength offsets are listed in Table 1. These offsets, consistent with those applied by L97, result in the absorption line positions matching the laboratory wavelengths (e.g., Savage et al. 1993; Morton 1991) to within $\pm 0.3 \AA$ ( $\mathrm{rms}$ ).

The spectra from the individual gratings were co-added to produce a full $1141-3278 \AA(1075-3090 \AA$ rest frame $)$ spectrum. The spectra from gratings $\mathrm{G} 190 \mathrm{H}$ and $\mathrm{G} 270 \mathrm{H}$ were obtained the same day and show no difference in the continuum level, and so were co-added without scaling either spectrum, using the average flux in the overlapping region. The continuum-level in the $\mathrm{G} 130 \mathrm{H}$ spectrum, taken 6 months earlier, is clearly shifted relative to that of G190H. The $\mathrm{G} 130 \mathrm{H}$ spectrum normalization is described in $\S 2.1$. For consistency, the combined, final spectrum was rebinned ${ }^{11}$ to match the dispersion of the $\mathrm{G} 270 \mathrm{H}$ grating data, $\Delta \lambda=0.511 \AA$ pixel $^{-1}$, the lowest dispersion available in the three gratings (Table 1). The spectrum ranges from 1075.2 to $3089.8 \AA$, has a dispersion of $0.482 \AA \mathrm{pixel}^{-1}$ and a resolution of $1.86 \AA$ in the rest frame (Leitherer 1995). The

\footnotetext{
${ }^{9}$ Note: Errors are not quoted by Stark et al. (1992), but the applied correction technique for stray radiation was developed by Lockman, Jahoda, \& McCammon (1986), who quote an error of $\sim 1 \times 10^{19} \mathrm{~cm}^{-2}$. Using the same correction technique Elvis, Lockman, \& Wilkes (1989) measure an $\mathrm{H}$ I column density toward I Zw 1 of $N_{\mathrm{H}}=5.07( \pm 0.1) \times 10^{20}$ $\mathrm{cm}^{-2}$ consistent with the Stark et al. measurement. The slight offset between the two $N_{\mathrm{H}}$ measurements will not significantly affect the reddening correction.

${ }^{10}$ IRAF is distributed by the National Optical Astronomy Observatories, which is operated by the Association of Universities for Research in Astronomy, Inc. (AURA), under cooperative agreement with the National Science Foundation.

${ }^{11}$ The rebinning is necessary in order to combine and simultaneously process the $\mathrm{G} 130 \mathrm{H}, \mathrm{G} 190 \mathrm{H}$, and $\mathrm{G} 270 \mathrm{H}$ spectra for generating the template. The resultant resolution is sufficient for the purpose of generating and applying the template.
} 
TABLE 2

ABSORPTION LINE IDENTIFICATIONS ${ }^{\mathrm{a}}$

\begin{tabular}{|c|c|c|c|c|c|c|c|c|c|c|}
\hline $\begin{array}{l}\text { Apparent }{ }^{\mathrm{b}} \\
\text { Feature and Position } \\
\text { (1) }\end{array}$ & $\begin{array}{l}\text { Ion } \\
(2)\end{array}$ & $\begin{array}{l}\lambda_{\text {lab }}^{\mathrm{c}} \\
(\AA) \\
(3)\end{array}$ & $\begin{array}{c}\lambda_{\text {obs }}^{\text {meas }} \\
(\AA) \\
(4)\end{array}$ & $\begin{array}{c}\lambda_{\text {rest }}^{\text {meas }} \\
(\AA) \\
(5)\end{array}$ & $\begin{array}{c}\Delta v^{\mathrm{d}} \\
\left(\mathrm{km} \mathrm{s}^{-1}\right) \\
(6)\end{array}$ & $\begin{array}{l}\text { Flux } \\
\text { (7) }\end{array}$ & $\begin{array}{l}\mathrm{EW} \\
(\AA) \\
(8)\end{array}$ & $\begin{array}{c}\text { FWHM } \\
\left(\mathrm{km} \mathrm{s}^{-1}\right) \\
(9)\end{array}$ & $\begin{array}{l}\text { Mult. } \\
\text { (10) }\end{array}$ & $\begin{array}{c}\text { Comments } \\
\text { (11) }\end{array}$ \\
\hline$\lambda 1109$ (a) ............ & $\mathrm{C} \mathrm{III \star}$ & $1175.5[1]$ & 1177.6 & 1109.8 & +523 & 1.57 & 0.4 & 125 & UV4 & \\
\hline \multirow[t]{4}{*}{$\lambda 1124$ (b) ............ } & $\mathrm{Si}$ II & $1190.4[2]$ & 1190.3 & 1121.8 & -25 & 2.17 & 0.6 & 260 & UV5 & \\
\hline & Si II & $1193.3[2]$ & 1192.7 & 1124.1 & -150 & 1.50 & 0.4 & 260 & UV5 & \\
\hline & Si II & $1194.5[1]$ & 1193.6 & 1125.0 & -226 & 1.09 & 0.3 & 260 & UV5 & \\
\hline & Si II & $1197.4[1]$ & 1195.3 & 1126.6 & -526 & 1.02 & 0.3 & 260 & UV5 & \\
\hline \multirow[t]{4}{*}{$\lambda 1135$ (c) ............ } & $\mathrm{N}_{\mathrm{I}}$ & $1200.0^{f}[1]$ & 1200.2 & 1131.2 & +50 & 2.90 & 0.8 & 595 & UV1 & $\lambda \lambda 1199.5,1200.2,1200.8$ \\
\hline & Mn II & $1203.8[1]$ & 1203.9 & 1134.7 & +25 & 1.58 & 0.4 & 570 & UV26? & \\
\hline & $\mathrm{S} \mathrm{I}^{\mathrm{g}}$ & $1205.6[1]$ & $\ldots$ & $\ldots$ & -420 & $\ldots$ & $\ldots$ & $\ldots$ & & Alt. ID \\
\hline & Si III & $1206.5[2]$ & 1205.7 & 1137.3 & -200 & 3.59 & 1.0 & 580 & UV2 & \\
\hline \multirow[t]{7}{*}{$\lambda 1145$ (d) ............ } & $\mathrm{Mn}$ II $^{\mathrm{g}}$ & $1210.6[1]$ & 1210.2 & 1140.6 & -105 & 4.70 & 1.3 & 770 & & \\
\hline & $\mathrm{Mn}$ II $^{\mathrm{g}}$ & $1213.3[1]$ & 1213.0 & 1143.3 & -65 & 2.51 & 0.7 & 725 & & \\
\hline & $\mathrm{S} \mathrm{I}^{\mathrm{g}}$ & $1214.3[1]$ & 1214.0 & 1144.2 & -75 & 6.80 & 1.9 & 1025 & & \\
\hline & $\operatorname{Ly} \alpha^{\mathrm{g}}$ & $1215.7[2]$ & 1215.5 & 1145.6 & -50 & 0.016 & 0.005 & 135 & UV1 & \\
\hline & $\mathrm{Ly} \alpha^{\mathrm{g}}$ & $1215.7[2]$ & 1217.4 & 1147.4 & +417 & 7.20 & 2.0 & 990 & UV1 & $z=0.0014$ \\
\hline & $\operatorname{Ly} \alpha^{\mathrm{g}}$ & $1215.7[2]$ & 1220.5 & 1150.3 & +1177 & 6.40 & 1.8 & 1065 & UV1 & $z=0.004$ \\
\hline & $\operatorname{Ly} \alpha^{\mathrm{g}}$ & $1215.7[2]$ & 1225.7 & 1155.2 & +2460 & 1.45 & 0.4 & 725 & UV1 & $z=0.0082$ \\
\hline$\lambda 1187$ (e) ............ & S II & $1259.5[2]$ & 1260.1 & 1187.7 & +155 & 2.69 & 0.8 & 325 & UV9 & \\
\hline \multirow[t]{3}{*}{$\lambda 1226(\mathrm{~g}) \ldots \ldots \ldots \ldots \ldots$} & $\mathrm{O}_{\mathrm{I}}$ & $1302.2[2]$ & 1302.1 & 1227.2 & -23 & 4.39 & 1.2 & 290 & UV2 & \\
\hline & Si II & $1304.4[2]$ & 1304.5 & 1229.5 & +23 & 4.23 & 1.2 & 290 & UV3 & \\
\hline & $N v^{h}$ & $1238.8[2]$ & 1306.0 & 1230.9 & -1913 & 5.23 & 1.5 & 290 & UV1 & $z_{\mathrm{abs}}=0.0064$ \\
\hline$\lambda 1234$ (h) $\ldots \ldots \ldots \ldots \ldots$ & $\mathrm{N} \mathrm{v}^{\mathrm{h}}$ & $1242.8[2]$ & 1310.2 & 1234.9 & -1907 & 6.15 & 1.7 & 340 & UV1 & \\
\hline \multirow[t]{2}{*}{$\lambda 1257$ (i) $\ldots \ldots \ldots \ldots \ldots$} & $\mathrm{C}_{\text {II }}$ & $1334.5[2]$ & 1334.3 & 1257.6 & -42 & 0.23 & 0.06 & 210 & UV1 & \\
\hline & $\mathrm{C} \mathrm{II}^{\star}$ & $1335.7[2]$ & 1334.5 & 1257.8 & -264 & 2.11 & 0.6 & 210 & UV1 & \\
\hline \multirow[t]{2}{*}{$\lambda 1317(\mathrm{j}) \ldots \ldots \ldots \ldots$} & Si IV & $1393.8[2]$ & 1394.0 & 1313.9 & +43 & 0.88 & 0.3 & 310 & UV1 & \\
\hline & Si IV & $1402.8[2]$ & 1403.2 & 1322.5 & +85 & 0.38 & 0.1 & 310 & UV1 & \\
\hline \multirow[t]{2}{*}{$\lambda 1440(\mathrm{k}) \ldots \ldots \ldots \ldots \ldots$} & Si II & $1526.7[2]$ & 1526.8 & 1439.0 & +19 & 2.22 & 0.6 & 495 & UV2 & \\
\hline & $\mathrm{Si}$ II & $1533.4[1]$ & 1533.6 & 1445.8 & +39 & 0.92 & 0.3 & 495 & UV2 & \\
\hline \multirow[t]{2}{*}{$\lambda 1460$ (1) $\ldots \ldots \ldots \ldots \ldots$} & $\mathrm{C}$ IV & $1548.2[2]$ & 1548.0 & 1459.0 & -39 & 2.35 & 0.7 & 570 & UV1 & \\
\hline & $\mathrm{C}$ IV & $1550.8[2]$ & 1551.2 & 1462.0 & +74 & 1.59 & 0.5 & 570 & UV1 & \\
\hline$\lambda 1575(\mathrm{~m}) \ldots \ldots \ldots \ldots \ldots$ & $\mathrm{Al}$ II & $1670.8[2]$ & 1670.8 & 1574.7 & 0 & 1.08 & 0.3 & 245 & UV2 & \\
\hline$\lambda 1703$ (n) ............ & Si II & $1808.0[2]$ & 1807.9 & 1704.0 & -15 & 0.45 & 0.1 & 220 & UV1 & \\
\hline \multirow[t]{2}{*}{$\lambda 1823$ (o) ............. } & $\mathrm{Fe}_{\mathrm{I}}$ & $1934.5[1]$ & 1934.1 & 1822.9 & -60 & 0.53 & 0.2 & 220 & UV37 & \\
\hline & $\mathrm{Fe}_{\mathrm{I}}$ & 1937.3[1] & 1936.4 & 1825.1 & -140 & 0.49 & 0.2 & 2190 & UV35 & \\
\hline$\lambda 1909$ (p) ............ & $\mathrm{Zn}$ II & $2026.1[2]$ & 2026.1 & 1909.6 & 0 & 0.97 & 0.3 & 180 & UV1 & \\
\hline \multirow[t]{6}{*}{$\lambda 2121$ (q) ............ } & $\mathrm{Fe}$ II & $2249.9[1]$ & 2248.8 & 2119.5 & -145 & 0.41 & 0.1 & 305 & UV5 & \\
\hline & $\mathrm{Fe} I$ & $2251.5[1]$ & 2250.7 & 2121.3 & -105 & 0.5 & 0.2 & 265 & UV16 & \\
\hline & $\mathrm{Ni} \mathrm{I}^{\mathrm{g}}$ & $2255.5[1]$ & 2253.7 & 2124.1 & -240 & 0.23 & 0.1 & 295 & UV34 & \\
\hline & $\mathrm{Ni} \mathrm{I}$ & $2256.6[1]$ & 2256.9 & 2127.1 & +40 & 0.23 & 0.1 & 280 & UV9 & \\
\hline & $\mathrm{Fe} I$ & $2260.2[1]$ & $\ldots$ & $\ldots$ & -400 & $\ldots$ & $\ldots$ & $\ldots$ & UV15 & Alt. ID \\
\hline & $\mathrm{Fe}$ II & $2260.8[2]$ & 2260.7 & 2130.7 & -15 & 0.22 & 0.1 & 285 & UV4 & \\
\hline \multirow[t]{2}{*}{$\lambda 2171(\mathrm{r}) \ldots \ldots \ldots \ldots \ldots$} & $\mathrm{Mn}$ II $^{\mathrm{g}}$ & $2299.7[1]$ & 2300.9 & 2168.6 & +155 & 0.23 & 0.1 & 280 & UV2 & \\
\hline & $\mathrm{Mn} \Pi^{\mathrm{g}}$ & $2305.7[1]$ & 2304.5 & 2172.0 & -155 & 0.42 & 0.2 & 280 & UV2 & \\
\hline \multirow[t]{2}{*}{$\lambda 2206$ (s) ............ } & $\mathrm{Ni} \mathrm{I}$ & $2338.2[1]$ & 2339.5 & 2205.0 & +165 & 0.51 & 0.2 & 290 & UV8 & \\
\hline & $\mathrm{Fe}$ II & $2344.2[2]$ & 2344.0 & 2209.2 & -30 & 1.34 & 0.5 & 290 & UV3 & \\
\hline \multirow[t]{2}{*}{$\lambda 2235(\mathrm{t}) \ldots \ldots \ldots \ldots \ldots$} & 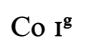 & $2365.8[1]$ & 2365.4 & 2229.4 & -50 & 0.29 & 0.1 & 295 & UV6 & \\
\hline & Cr I & $2365.5[1]$ & $\ldots$ & $\ldots$ & -10 & $\ldots$ & $\ldots$ & $\ldots$ & $\ldots$ & Alt. ID \\
\hline
\end{tabular}


TABLE 2-Continued

\begin{tabular}{|c|c|c|c|c|c|c|c|c|c|c|}
\hline $\begin{array}{l}\text { Apparent }^{\mathrm{b}} \\
\text { Feature and Position } \\
\text { (1) }\end{array}$ & $\begin{array}{l}\text { Ion } \\
(2)\end{array}$ & $\begin{array}{l}\lambda_{\text {lab }} \mathrm{c} \\
(\AA) \\
(3)\end{array}$ & $\begin{array}{l}\lambda_{\text {obs }}^{\text {meas }} \\
(\AA) \\
(4)\end{array}$ & $\begin{array}{c}\lambda_{\text {rest }}^{\text {meas }} \\
(\AA) \\
(5)\end{array}$ & $\begin{array}{c}\Delta v^{\mathrm{d}} \\
\left(\mathrm{km} \mathrm{s}^{-1}\right) \\
(6)\end{array}$ & $\begin{array}{c}\text { Flux }^{e} \\
\text { (7) }\end{array}$ & $\begin{array}{l}\text { EW } \\
(\AA) \\
(8)\end{array}$ & $\begin{array}{c}\text { FWHM } \\
\left(\mathrm{km} \mathrm{s}^{-1}\right) \\
(9)\end{array}$ & $\begin{array}{l}\text { Mult. } \\
\text { (10) }\end{array}$ & $\begin{array}{c}\text { Comments } \\
\text { (11) }\end{array}$ \\
\hline & $\mathrm{Al}$ I & $2367.8[1]$ & 2368.3 & 2232.1 & +60 & 0.48 & 0.2 & 295 & UV4 & \\
\hline & $\mathrm{Al}$ I & $2373.9^{\mathrm{f}}[1]$ & 2372.0 & 2235.6 & -240 & 0.26 & 0.1 & 295 & UV4 & \\
\hline & $\mathrm{Al}_{\mathrm{I}}$ & $2372.8[1]$ & $\ldots$ & $\ldots$ & -100 & $\ldots$ & $\ldots$ & $\ldots$ & UV3 & Alt. ID \\
\hline & $\mathrm{Fe}$ II & $2374.5[2]$ & 2374.5 & 2238.0 & 0 & 0.92 & 0.4 & 295 & UV2 & \\
\hline \multirow[t]{2}{*}{$\lambda 2245(\mathrm{u}) \ldots \ldots \ldots \ldots \ldots$} & $\mathrm{Fe}$ II & $2382.8[2]$ & 2382.9 & 2245.9 & +10 & 1.49 & 0.6 & 230 & UV2 & \\
\hline & Co I & $2385.6[1]$ & 2386.0 & 2248.8 & +50 & 0.2 & 0.1 & $145^{\mathrm{i}}$ & UV6 & \\
\hline$\lambda 2268$ (v) ............ & $\mathrm{Co} \mathrm{I}^{\mathrm{g}}$ & $2408.0[1]$ & 2407.6 & 2269.2 & -50 & 0.48 & 0.2 & $245^{\mathrm{i}}$ & UV6 & \\
\hline \multirow[t]{2}{*}{$\lambda 2445(\mathrm{w}) \ldots \ldots \ldots \ldots$} & $\mathrm{Fe}$ II & $2586.7[2]$ & 2586.9 & 2438.2 & -25 & 0.4 & 0.2 & $155^{\mathrm{i}}$ & UV1 & \\
\hline & $\mathrm{Fe}$ II & $2600.2[2]$ & 2600.1 & 2450.6 & -10 & 1.84 & 0.8 & $285^{\mathrm{i}}$ & UV1 & \\
\hline \multirow[t]{2}{*}{$\lambda 2635(x, y) \ldots \ldots \ldots \ldots$} & $\mathrm{Mg}$ II & $2796.4[2]$ & 2796.4 & 2635.6 & 0 & 2.15 & 1.0 & 215 & UV1 & \\
\hline & $\mathrm{Mg}$ II & $2803.5[2]$ & 2803.7 & 2642.5 & +20 & 2.14 & 1.0 & 215 & UV1 & \\
\hline$\lambda 2688(\mathrm{z}) \ldots \ldots \ldots \ldots \ldots$ & $\mathrm{Mg}_{\mathrm{I}}$ & $2853.0[2]$ & 2853.1 & 2689.1 & +15 & 0.61 & 0.3 & 215 & UV1 & \\
\hline
\end{tabular}

${ }^{\text {a }}$ All the absorption is consistent with Galactic (ISM) absorption, except where noted; see " $h$ ". C III ${ }^{\star}$ and C II ${ }^{\star}$ are metastable transitions.

${ }^{\text {b }}$ A simultaneous fit was made to each line (in col. [2]) listed as a Feature (in col. [1]) unless otherwise noted. Note, the wavelength of each Feature refers to the wavelength as it appears in the rest frame spectrum in Fig. 1.

c (1) Morton 1991; (2) Savage et al. 1993.

d $\Delta v$ is the line peak velocity shift from the expected rest frame position. The resolution is $470 \mathrm{~km} \mathrm{~s}^{-1}$ at $1200 \AA, 355 \mathrm{~km} \mathrm{~s}^{-1}$ at $1600 \AA, 280 \mathrm{~km}$ $\mathrm{s}^{-1}$ at $2000 \AA, 240 \mathrm{~km} \mathrm{~s}^{-1}$ at $2400 \AA$ and $200 \mathrm{~km} \mathrm{~s}^{-1}$ at $2800 \AA$ (Leitherer 1995).

e Units of $10^{-14} \mathrm{ergs} \mathrm{cm}^{-2} \mathrm{~s}^{-1}$.

${ }^{\mathrm{f}}$ Multiplet wavelength or mean wavelength of more than one transition. If more than two transitions, the wavelengths will be listed in the "Comments" column.

$\mathrm{g}$ The line identification is uncertain.

${ }^{\text {h }}$ Not Galactic absorption. This is absorption intrinsic to the object. Laor et al. 1997b identified these absorption features as UV (N v) Quasar associated absorption.

${ }^{i}$ A simultaneous fit was not possible due to the relative locations of the absorption lines.

I Zw 1 spectrum is shown in L97 and Figure 1 (dotted line). A redshift of $z=0.061$, defined by the $\mathrm{Mg}$ II profile peak, is used throughout this paper and is consistent with measurements by Phillips (1976), Schmidt \& Green (1983), Wu et al. (1983), Persson \& McGregor (1985), van Groningen (1993), Condon et al. (1985) and Smith et al. (1997). Condon et al. determine a redshift of 0.061136 to an accuracy of $8 \times 10^{-6}$ using $\mathrm{H}$ I radio measurements.

The spectral energy distributions of quasars and AGNs (Elvis et al. 1994) span 11 orders of magnitude in frequency from X-rays to millimeter wavelengths (and to radio wavelengths for some objects; Weedman 1986; Peterson 1997). Observations (e.g., Oke, Shields, \& Korycansky 1984; Wills et al. 1985) suggest that the continuum at optical and UV wavelengths can be approximated by a single power-law, $F_{v} \sim v^{-\alpha_{v}}$. We thus chose to fit a power-law continuum to the I $\mathrm{Zw} 1$ rest frame spectrum before any emission line features were fitted. After the initial completion of the iron template, which included careful fittings (\$ 3.3.2) of the emission lines which are not iron, it was realized that due to an STSDAS software bug ${ }^{12}$ the archival G130H spectrum was unknowingly calibrated with the wrong (pre-costar) flux calibration files. These data were then recalibrated ${ }^{13}$ using the most recent (1996 March) calibration files. We then fitted a power-law continuum from 1075 to $\sim 1720 \AA$, inde-

\footnotetext{
12 The relevant task, "ADDNEWKEYS," has now been updated by the STScI HST help desk.

13 This recalibration results in a flux level $\sim 9 \%$ higher than that of L97, because the FOS calibration files were updated (1996 March) later than their data processing in 1994.
}

pendent of the power-law continuum at longer wavelengths. ${ }^{14}$ The resulting continuum is a broken power-law with the break at $1716 \AA$ : a blue continuum slope, $\alpha_{v}=1.9$ and normalization, $F_{\lambda}(1500 \AA)=3.45 \times 10^{-14} \mathrm{ergs} \mathrm{cm}^{-2}$ $\mathrm{s}^{-1} \AA^{-1}$, and a red continuum slope, $\alpha_{v}=1.0$ and $F_{\lambda}(1500$ $\AA)=3.89 \times 10^{-14} \mathrm{ergs} \mathrm{cm}^{-2} \mathrm{~s}^{-1} \AA^{-1}$. The choice of this continuum (Fig. 1) does not affect the application of the template, as it will be scaled and broadened to match the target spectrum in the application process (§ 4). The continuum windows used in the fitting (1312-1327, 1347-1353, $1641-1647,1675-1690$, and $3007-3027 \AA$ ) are specific to the I $\mathrm{Zw} 1$ spectrum and are different from those suggested by Francis et al. (1991), which were based on an average AGN spectrum. The spectrum of $\mathrm{I} Z \mathrm{w} 1$ is rich in low-ionization lines and weak emission features (Fig. 1; L97) which contaminate the "average" continuum windows.

\subsection{The G130H Spectrum Scaling and I Zw 1 Variability}

The variation in continuum level between the $\mathrm{G} 130 \mathrm{H}$ and the $\mathrm{G} 190 \mathrm{H}+\mathrm{G} 270 \mathrm{H}$ spectra taken 6 months apart is consistent with the known variability of I Zw 1 (e.g., Zwicky 1971; Giannuzzo \& Stirpe 1996; Rodríguez-Pascual et al. 1997; Leighly 1999). The normalization of the recalibrated G130H spectrum to the $\mathrm{G} 190 \mathrm{H}+\mathrm{G} 270 \mathrm{H}$ spectrum was determined as the ratio of the median flux in the overlapping region of the spectra $(1.3 \pm 0.56)$. As the bluest $\sim 40 \AA$ of the $\mathrm{G} 190 \mathrm{H}$ spectrum shows a relative error of $42 \%$ as

\footnotetext{
${ }^{14}$ The emission and absorption models below $1700 \AA$ A were then regenerated, and new iron templates (of Fe II and Fe III emission) were constructed.
} 


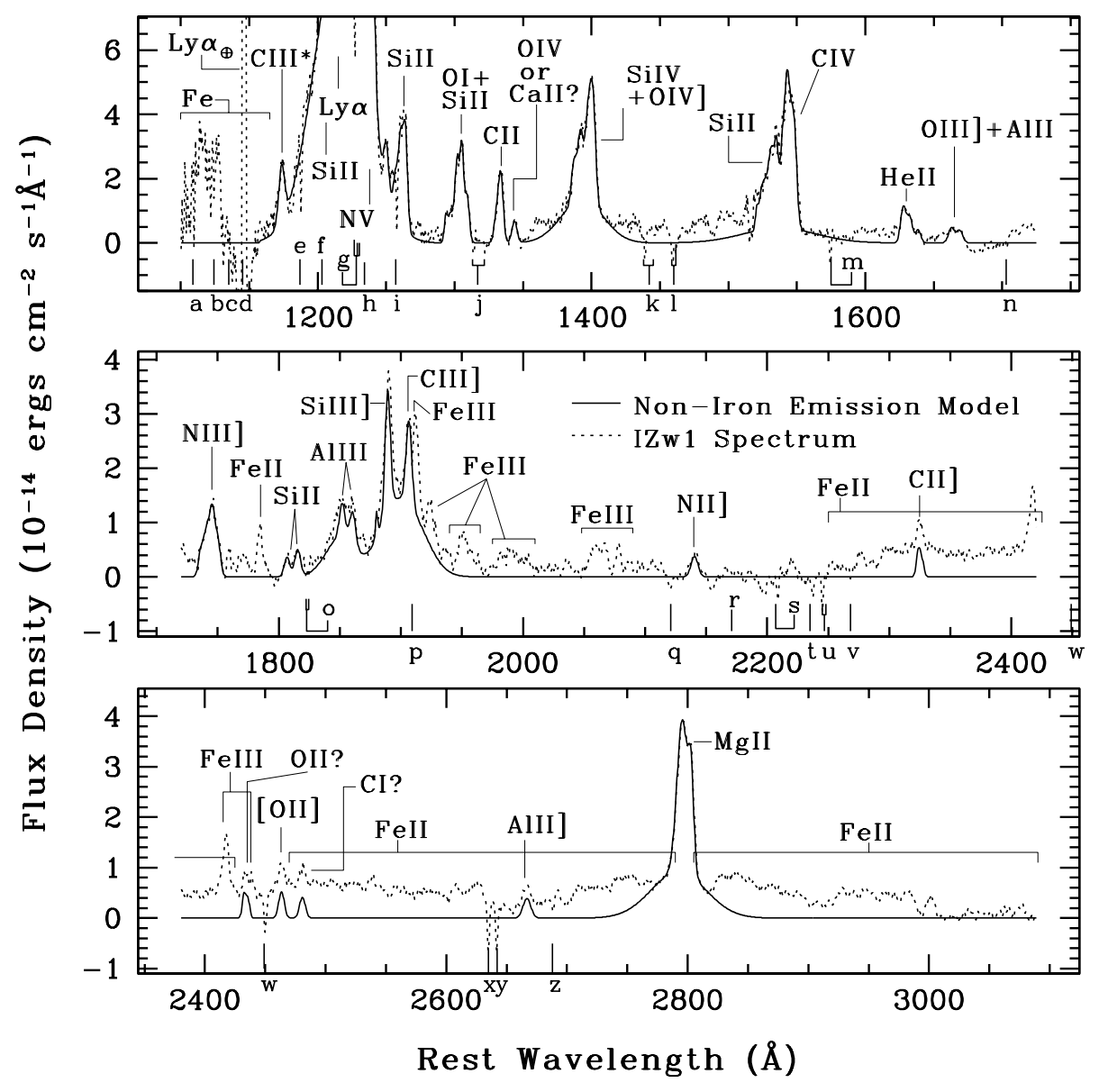

FIG. 1.-Non-iron emission model (solid line) overplotted on the continuum-subtracted I Zw 1 spectrum (dotted line). The assumed continuum of the HST spectrum is a broken power law, $F_{v} \sim v^{-\alpha}$ with a break at $1716 \AA$ (see text for details). The absorption lines are marked using letters a-z below the spectrum and listed in Table 2 . The detected emission lines are labeled. Geocoronal Ly $\alpha$ is seen at $1145 \AA$ (rest frame).

opposed to $4 \%$ in $\mathrm{G} 130 \mathrm{H}$, the normalized $\mathrm{G} 130 \mathrm{H}$ spectrum substituted that of $\mathrm{G} 190 \mathrm{H}$ in this overlapping region. However, the G190H flux uncertainty entirely dominates the formal error on the normalization, and so useful line flux estimates cannot be deduced below $1500 \AA$ A In spite of the large formal error, a smooth spectrum and power-law continuum resulted in the 1075-1716 $\AA$ region, implying that the actual uncertainty is lower. As the template is best applied by subdividing the 1075-3090 $\AA$ range before scaling to match the iron emission strength in individual quasar spectra (§§ 4.2-4.3), its use is not adversely affected by the $\mathrm{G} 130 \mathrm{H}$ normalization. In fact, the poor match of the $\mathrm{Fe}$ II emission in the G130H grating spectrum for some objects (see $\S 4.3$ ) directly shows the need to subdivide the template in order to obtain optimum fits to AGN spectra. This is because all the individual iron multiplets did not brighten with the same factor in I $\mathrm{Zw} 1$ as did the continuum in the time between the $\mathrm{G} 130 \mathrm{H}$ and the $\mathrm{G} 190 \mathrm{H}$ spectra were observed. If iron multiplet strengths vary in the same object, they are also very likely to vary among objects. This is also evident from the fact that not all AGNs have Fe II UV191, Fe III UV34, and/or Fe III UV47 multiplets as strong as does I Zw 1 ( 4.3 and Appendix A). Lanzetta et al. (1993) present a spectrum of I Zw 1 representing the average of the UV spectra observed with the International Ultraviolet Explorer (IUE) from 1978 through 1982. Comparison with the HST data to confirm the scaling of the $\mathrm{G} 130 \mathrm{H}$ spectrum is, however, not possible due to the widely differing host galaxy contribution ${ }^{15}$ and to line and continuum variations (cf. Wu et al. 1983; Pian \& Treves 1993; Wang et al. 1996; Rodríguez-Pascual et al. 1997; L97).

The only other UV data available are, to our knowledge, spectropolarimetric data in the HST data archives. A comparison with such data requires knowledge of the scattering medium and an understanding of the scattered spectrum, which are beyond the scope of this work. Hence, it is not possible at present to constrain the absolute scaling of $\mathrm{G} 130 \mathrm{H}$ spectrum further, though as noted this uncertainty does not adversely affect the applicability of the iron template.

\section{THE IRON EMISSION TEMPLATE}

\subsection{Development of the Template}

Once the data were calibrated, rebinned to a common dispersion, and co-added the template was generated using the following procedure:

1. A power-law continuum was fitted to pure continuum wavelength regions $(\S 2)$ in the spectrum, and this continuum fit was subtracted (result is overplotted in Fig. 1, dotted line).

\footnotetext{
15 The IUE spectrograph has a large aperture $\left(10^{\prime \prime} \times 20^{\prime \prime}\right.$, Boggess et al. 1978) thereby sampling the AGN host galaxy.
} 
TABLE 3

Emission Line Fits and Measurements

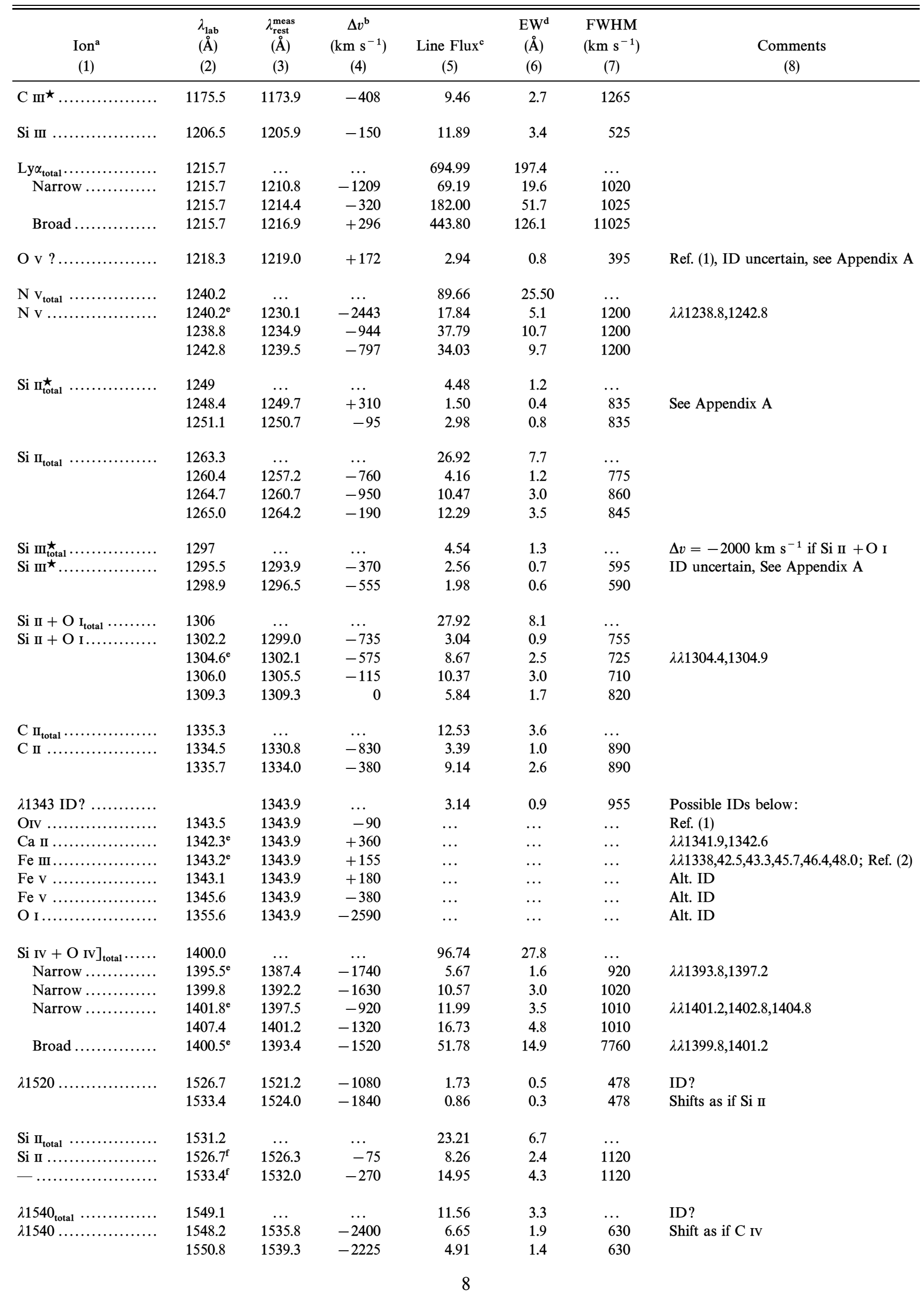


TABLE 3-Continued

\begin{tabular}{|c|c|c|c|c|c|c|c|}
\hline $\begin{array}{c}\text { Ion }^{a} \\
(1)\end{array}$ & $\begin{array}{l}\lambda_{\text {lab }} \\
(\AA) \\
(2)\end{array}$ & $\begin{array}{c}\lambda_{\text {rest }}^{\text {meas }} \\
(\AA) \\
(3)\end{array}$ & $\begin{array}{c}\Delta v^{\mathrm{b}} \\
\left(\mathrm{km} \mathrm{s}^{-1}\right) \\
(4)\end{array}$ & $\begin{array}{l}\text { Line Flux } \\
\text { (5) }\end{array}$ & $\begin{array}{c}\mathrm{EW}^{\mathrm{d}} \\
(\AA) \\
(6)\end{array}$ & $\begin{array}{c}\text { FWHM } \\
\left(\mathrm{km} \mathrm{s}^{-1}\right) \\
(7)\end{array}$ & $\begin{array}{c}\text { Comments } \\
\text { (8) }\end{array}$ \\
\hline $\mathrm{C} \mathrm{IV}_{\text {total }} \ldots \ldots \ldots \ldots \ldots$ & 1549.1 & $\ldots$ & $\ldots$ & 74.68 & 21.9 & $\ldots$ & \\
\hline $\mathrm{C}$ IV narrow ............ & $1548.2^{\mathrm{f}}$ & 1543.3 & -950 & 29.11 & 8.6 & 1100 & Best fit \\
\hline 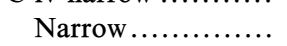 & $1550.8^{\mathrm{f}}$ & 1548.4 & -465 & 14.93 & 4.4 & 895 & \\
\hline Broad ............... & 1549.1 & 1538.1 & -2130 & 30.64 & 8.9 & 12265 & \\
\hline $\mathrm{C}$ IV narrow ............ & $1548.2^{\mathrm{f}}$ & 1543.3 & -950 & 27.73 & 8.1 & 1070 & See Appendix A \\
\hline (Alternative fits) ....... & $1550.8^{\mathrm{f}}$ & 1548.4 & -465 & 14.10 & 4.1 & 1070 & \\
\hline$-\ldots \ldots \ldots \ldots \ldots \ldots \ldots$ & $\ldots$ & 1547.9 & $\ldots$ & 2.29 & 0.7 & 535 & Fit to residual \\
\hline C IV complex ${ }^{\mathrm{g}} \ldots \ldots \ldots$ & $\sim 1548$ & $\cdots$ & $\ldots$ & 126.78 & 36.47 & $\ldots$ & $\lambda \lambda 1505-1560$ (Incl. iron) emission \\
\hline $\mathrm{He} \mathrm{II}_{\text {total }} \ldots \ldots \ldots \ldots \ldots$ & 1640.4 & $\ldots$ & $\ldots$ & 11.24 & 3.3 & $\ldots$ & \\
\hline \multirow{2}{*}{ He iI .................... } & $1640.4^{\mathrm{a}}$ & 1628.4 & -2195 & 5.38 & 1.6 & 840 & \\
\hline & $1640.4^{\mathrm{a}}$ & 1633.1 & -1335 & 3.92 & 1.1 & 840 & \\
\hline He II................ & $1640.4^{\mathrm{f}}$ & 1639.2 & -220 & 1.94 & 0.6 & 820 & \\
\hline $\mathrm{O} \mathrm{III}] \ldots \ldots \ldots \ldots \ldots \ldots$ & $1664.2^{\mathrm{a}}$ & 1663.7 & -90 & 2.61 & 0.8 & 940 & Fit with $\mathrm{Al}$ II \\
\hline $\mathrm{Al}$ II $\ldots \ldots \ldots \ldots \ldots \ldots$ & $1670.8^{\mathrm{a}}$ & 1669.7 & -200 & 2.15 & 0.5 & 940 & Fit with $\mathrm{O}$ III] \\
\hline $\mathrm{N} \mathrm{IIII}]_{\text {total }} \ldots \ldots \ldots \ldots \ldots$ & 1750.5 & $\ldots$ & $\ldots$ & 16.85 & 4.9 & $\ldots$ & \\
\hline \multirow{5}{*}{$\mathrm{N}$ III] $] \ldots \ldots \ldots \ldots \ldots \ldots$} & 1746.8 & 1737.0 & -1685 & 2.47 & 0.7 & 830 & \\
\hline & 1748.7 & 1741.0 & -1320 & 3.16 & 0.9 & 830 & \\
\hline & 1749.7 & 1744.0 & -975 & 2.82 & 0.8 & 830 & \\
\hline & 1752.2 & 1746.8 & -925 & 5.25 & 1.6 & 830 & \\
\hline & 1754.0 & 1751.0 & -575 & 3.15 & 0.9 & 830 & \\
\hline $\mathrm{Si}_{\mathrm{II}_{\text {total }} \ldots \ldots \ldots \ldots \ldots \ldots}$ & 1814.0 & $\ldots$ & $\ldots$ & 4.98 & 1.5 & $\ldots$ & \\
\hline \multirow[t]{2}{*}{$\mathrm{Si}$ II ................... } & $1808.0^{\mathrm{f}}$ & 1807.6 & -65 & 2.06 & 0.6 & 885 & \\
\hline & $1817.2^{\mathrm{f}}$ & 1816.1 & -180 & 2.92 & 0.9 & 925 & \\
\hline $\mathrm{Al} \mathrm{III}_{\text {total }} \ldots \ldots \ldots \ldots \ldots$ & 1857 & . & $\ldots$ & 30.10 & 9.6 & $\ldots$ & \\
\hline Narrow.............. & 1854.4 & 1852.7 & -275 & 3.50 & 1.1 & 870 & \\
\hline Narrow ............... & 1862.8 & 1861.3 & -240 & 3.65 & 1.2 & 910 & \\
\hline \multirow[t]{2}{*}{ Broad .............. } & 1854.4 & 1849.1 & -860 & 13.09 & 4.2 & 4530 & \\
\hline & 1862.8 & 1856.0 & -1095 & 9.86 & 3.1 & 4510 & \\
\hline$[\mathrm{Si}$ III $] \ldots \ldots \ldots \ldots \ldots \ldots$ & 1882.7 & 1880.3 & -380 & 1.02 & 0.3 & 340 & Fe II $\lambda 1881 ?$, Model B \\
\hline $\mathrm{Si} \mathrm{IIII}]_{\text {total }} \ldots \ldots \ldots \ldots \ldots$ & 1892.0 & $\ldots$ & $\ldots$ & 29.25 & 9.6 & $\ldots$ & Model B \\
\hline 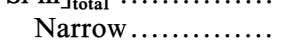 & 1892.0 & 1889.5 & -400 & 11.80 & 3.9 & 800 & \\
\hline Broad $^{\mathrm{a}} \ldots \ldots \ldots \ldots \ldots$ & 1892.0 & 1889.5 & -400 & 17.45 & 5.7 & 3950 & Fit with $\mathrm{C}$ III] \\
\hline $\mathrm{C}$ III $]_{\text {total }} \ldots \ldots \ldots \ldots \ldots$ & 1908.7 & $\ldots$ & $\ldots$ & 51.85 & 17.0 & $\ldots$ & Model B \\
\hline Narrow............. & 1908.7 & 1906.8 & -300 & 9.24 & 3.0 & 880 & \\
\hline $\operatorname{Broad}^{\mathrm{a}} \ldots \ldots \ldots \ldots \ldots$ & 1908.7 & 1906.8 & -300 & 42.61 & 14.0 & 5500 & Fit with Si III] \\
\hline $\mathrm{C}$ III] complex I....... & $\sim 1909$ & $\ldots$ & $\ldots$ & 82.12 & 26.9 & $\ldots$ & $\mathrm{C}$ III $]+\mathrm{Si} \mathrm{IIII}]+[\mathrm{Si} \mathrm{III}]$ \\
\hline $\mathrm{C}$ III] complex II ...... & $\sim 1909$ & $\ldots$ & $\ldots$ & 112.22 & 36.5 & $\ldots$ & $\mathrm{C}$ III $]+\mathrm{Si}$ III $]+\left[\mathrm{Si}_{\mathrm{III}}\right]+\mathrm{Al} \mathrm{III}$ \\
\hline $\mathrm{N}$ iI $] \ldots \ldots \ldots \ldots \ldots \ldots$ & 2141.4 & 2141.0 & -55 & 2.66 & 1.0 & 935 & \\
\hline $\mathrm{C}$ II $]_{\text {total }} \ldots \ldots \ldots \ldots \ldots$ & 2326.6 & $\ldots$ & $\ldots$ & 3.11 & 1.2 & $\ldots$ & \\
\hline \multirow{4}{*}{$\mathrm{C}$ II $] \ldots \ldots \ldots \ldots \ldots \ldots \ldots$} & 2325.4 & 2324.0 & -180 & 0.95 & 0.4 & 425 & \\
\hline & 2326.1 & 2325.6 & -65 & 1.05 & 0.4 & 425 & \\
\hline & 2327.7 & 2327.2 & -65 & 0.55 & 0.2 & 425 & \\
\hline & 2328.8 & 2328.7 & -10 & 0.56 & 0.2 & 425 & \\
\hline \multirow[t]{3}{*}{$\lambda 2435$ ID? ............ } & $\ldots$ & 2435.4 & $\ldots$ & 3.18 & 1.34 & $\ldots$ & Model sum \\
\hline & $\ldots$ & 2436.8 & $\ldots$ & 1.40 & 0.59 & 430 & Modeled \\
\hline & $\ldots$ & 2433.5 & $\ldots$ & 1.78 & 0.75 & 430 & Modeled \\
\hline $\mathrm{O}_{\text {II }} \ldots \ldots \ldots \ldots \ldots \ldots$ & $2440.7^{e}$ & 2435.4 & -650 & $\ldots$ & $\ldots$ & $\ldots$ & Suggested ID, Ref. (3) \\
\hline 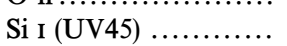 & 2435.2 & 2435.4 & +25 & $\cdots$ & $\cdots$ & $\cdots$ & Alt. ID, Ref. (3) \\
\hline Si I (UV2).............. & $2444.3^{\mathrm{e}}$ & 2435.4 & -1090 & $\ldots$ & $\ldots$ & $\ldots$ & Alt. ID, Ref. (3) \\
\hline$[\mathrm{O}$ II $] \ldots \ldots \ldots \ldots \ldots$ & 2470.3 & 2464.6 & -690 & 3.05 & 1.0 & 665 & Ref. (1) \\
\hline & & & & 9 & & & \\
\hline
\end{tabular}


TABLE 3-Continued

\begin{tabular}{|c|c|c|c|c|c|c|c|}
\hline $\begin{array}{c}\text { Ion }^{a} \\
\text { (1) }\end{array}$ & $\begin{array}{l}\lambda_{\text {lab }} \\
(\AA) \\
(2)\end{array}$ & $\begin{array}{c}\lambda_{\text {rest }}^{\text {meas }} \\
(\AA) \\
(3)\end{array}$ & $\begin{array}{c}\Delta v^{\mathrm{b}} \\
\left(\mathrm{km} \mathrm{s} \mathrm{s}^{-1}\right) \\
(4)\end{array}$ & $\begin{array}{l}\text { Line Flux } \\
\text { (5) }\end{array}$ & $\begin{array}{l}\mathrm{EW}^{\mathrm{d}} \\
(\AA) \\
(6)\end{array}$ & $\begin{array}{c}\text { FWHM } \\
\left(\mathrm{km} \mathrm{s}^{-1}\right) \\
(7)\end{array}$ & $\begin{array}{c}\text { Comments } \\
(8)\end{array}$ \\
\hline $\mathrm{C}_{\mathrm{I}} ? \ldots \ldots \ldots \ldots \ldots$ & 2478.6 & 2482.0 & +410 & 2.52 & 1.1 & 690 & ID uncertain, Ref. (3) \\
\hline $\mathrm{Al}$ II ................. & 2670.0 & 2667.8 & -245 & 3.14 & 1.5 & 860 & \\
\hline$M g \mathrm{II}_{\text {total }} \ldots \ldots \ldots \ldots$ & $2797.9^{\mathrm{e}}$ & $\ldots$ & $\ldots$ & 94.31 & 45.8 & $\ldots$ & $\lambda \lambda 2795.5,2802.7$ \\
\hline \multirow[t]{4}{*}{ Narrow ........... } & 2795.5 & 2796.2 & $+75^{\mathrm{h}}$ & 12.13 & 5.9 & 640 & 4 Gauss fit \\
\hline & 2802.7 & 2803.4 & $+75^{\mathrm{h}}$ & 12.13 & 5.9 & 640 & Ditto; doublet at rest \\
\hline & 2795.5 & 2792.0 & -375 & 9.06 & 4.4 & 790 & Ditto; shifted doublet \\
\hline & 2802.7 & 2799.4 & -350 & 9.06 & 4.4 & 790 & Ditto \\
\hline Broad $\ldots . . . \ldots \ldots$ & $2797.9^{\mathrm{e}}$ & 2793.1 & -515 & 51.94 & 25.2 & 5720 & $\lambda \lambda 2795.5,2802.7$ \\
\hline Mg II narrow ....... & 2795.5 & 2796.3 & $+85^{\mathrm{h}}$ & 33.84 & 16.5 & 1130 & 2 Gauss fit; for $z$ \\
\hline (Alternative fit)...... & 2802.7 & 2803.5 & $+85^{\mathrm{h}}$ & 8.98 & 4.4 & 550 & measurement \\
\hline
\end{tabular}

${ }^{a}$ Each line/blend component, identified by element name in col. (1), was fitted separately from the other (named) components - unless otherwise noted. Unnamed components following a named one were fitted simultaneously with this component. See also Appendix A for further details on each fitted feature.

${ }^{\mathrm{b}} \Delta v$ is the line peak velocity shift from the expected rest frame position. The resolution is $470 \mathrm{~km} \mathrm{~s}^{-1}$ at $1200 \AA, 355 \mathrm{~km}$ $\mathrm{s}^{-1}$ at $1600 \AA, 280 \mathrm{~km} \mathrm{~s}^{-1}$ at $2000 \AA, 240 \mathrm{~km} \mathrm{~s}^{-1}$ at $2400 \AA$, and $200 \mathrm{~km} \mathrm{~s}^{-1}$ at $2800 \AA$ (Leitherer 1995).

${ }^{c}$ Units of $10^{-14} \mathrm{ergs} \mathrm{cm}^{-2} \mathrm{~s}^{-1}$.

${ }^{\mathrm{d}}$ Some line fits may have required a local continuum level different from the global continuum setting, but the EW measurements are always with respect to the global continuum.

e Multiplet wavelength or mean wavelength of more than one transition.

${ }^{f}$ A separate fit was made to each component; nonsimultaneous from the other transitions.

g The C IV complex contains all the emission above the continuum from 1505 to $1560 \AA$ : $1505-1525 \AA$ (incl. iron) emission, Si II $\lambda \lambda 1526,1533$, the $\lambda 1540$ feature, and the broad and narrow components of C IV.

${ }^{\mathrm{h}}$ Insignificant velocity shift. This shift is due to the profile fitting uncertainty and is not a real effect.

REFERENCES.-Wilkes 2000, and Morton 1991 unless specifically marked: (1) Penston et al. 1983; (2) Kurucz 1997; (3) Moore 1950.

2. All absorption and (non-iron and iron) emission features were identified, including the strong, weak, blended, and unblended ones (Fig. 1, Tables 2 and 3; $\S \S 3.2,3.3 .1$ ).

3. The non-iron emission features (Fig. 5) and Galactic/ ISM absorption features ( $\S 3.2,3.3 .2$ ) were fitted. This required a simultaneous fitting of some $\mathrm{Fe}$ III features (Table 4). Separate absorption and emission models were created (Fig. 1).

4. The remaining Fe III features were fitted and deblended, as needed (Table 4). The Fe III line emission was isolated, and an Fe III model was created (\$ 3.3.2).

5. The absorption and non-iron emission models were subtracted from the original spectrum to create an iron template (containing both Fe II and Fe III emission; Fig. 2).

6. The Fe III emission model was subtracted to create a pure Fe II template (Fig. 3).

7. The pixel values were set to 0 in the two iron templates in regions containing pure noise residuals left over from subtracting emission and absorption feature fits (Figs. 2 and 3). This prevents introduction of artifacts and noise to the target spectra when the template is applied.

In the following sections we discuss the fitting and identification steps of this procedure in more detail. We also compare the template, in $\S 3.4$, with other UV iron templates and theoretical models, currently available.

\subsection{Absorption Lines}

Features were identified as absorption lines (Table 2) when the minimum flux in the feature deviated by more than $3 \sigma(\sigma=\mathrm{rms}$ of the fluxes around the average local spectrum level) from the continuum or if the position of the feature coincided with that expected as part of a doublet line where the strongest line component was already identified. The identification of the specific ion and transition responsible for each absorption line was made using the list of typical ISM absorption lines by Savage et al. (1993) and the line lists by Morton (1991). All the absorption lines detected and identified in the spectrum are consistent with Galactic absorption with the exception of the features at 1306 and $1310 \AA$ which are due to $\mathrm{N} v \lambda \lambda 1238,1243$ (blueshifted) absorption associated with I Zw 1 (L97).

Absorption features were fitted by multiple Gaussian components assuming a constant width for all components of a multiplet. The fit was then subtracted from the spectrum.

\subsection{Emission Lines}

3.3.1. Line Identifications

Our identifications of each emission feature are based on reference line lists for non-iron (Wilkes 2000; Morton 1991; Verner, Barthel, \& Tytler 1994), and iron transitions [Moore 1950 ( $\mathrm{Fe}$ II and Fe III multiplets); Penston et al. 1983 (Fe II); Fuhr et al. 1988 (Fe II and Fe III); Giridhar \& Arellano Ferro 1995 (Fe II); Nahar 1995 (Fe II); Nahar \& Pradhan 1996 (Fe III); Kurucz 1997 (Fe II and Fe III); D. Verner (1996, private communication, Fe II)]. L97 suggested identification of a large number of features in the I $\mathrm{Zw} 1$ spectrum, but that paper was not our main reference as it appeared after our work was commenced and after most of the line identifications were completed. The data from L97 are essentially the same data presented here. Slight differences exist as this $\mathrm{G} 130 \mathrm{H}$ spectrum is recalibrated with more recent files and is renormalized to the level of the 


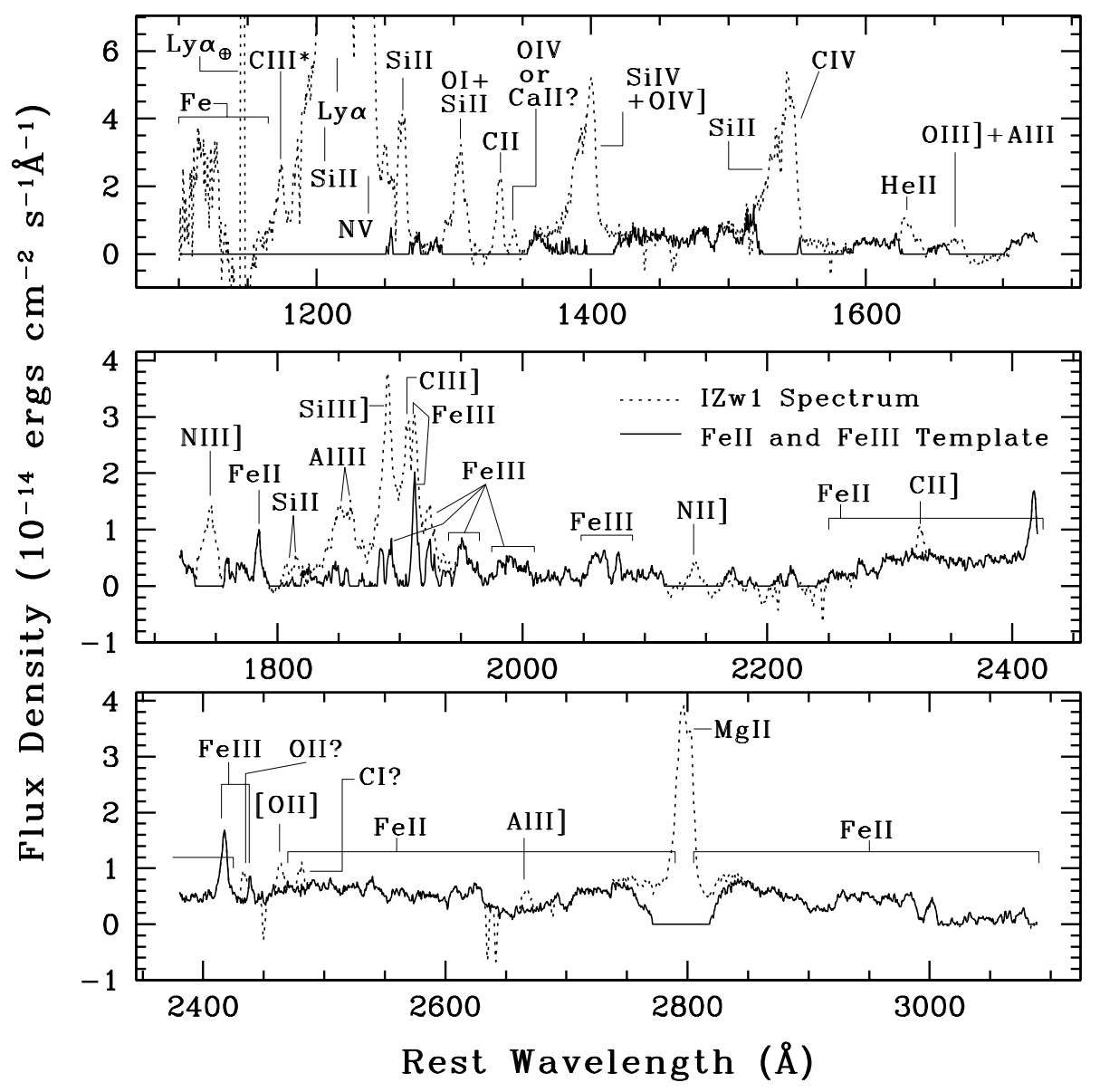

FIG. 2.-Iron template (solid line; sum of Fe II and Fe III) overplotted on the I Zw 1 spectrum (dotted line). The detected emission lines are labeled.

G190H spectrum (see § 2). Certain differences are present between our work and that by L97: (1) we identify a few additional non-iron features, (2) we suggest identifications of individual iron transitions in addition to the multiplet identifications in L97, (3) we find slightly different velocity shifts of the various line groups, and (4) many of the L97 line measurements deviate, though by no more than $30 \%$ for the stronger and/or isolated lines. We briefly discuss (3) and (4) in $\S \S 5.1 .2$ and 5.1.3.

In Appendix A we comment on the individual line features and their fits. A thorough discussion of line intensities is given by $\mathrm{L} 97$ and not repeated here apart from brief discussions of the weak $\mathrm{C} \mathrm{III]}$ ( $(5.2)$ and the relatively strong $1400 \AA ̊$ emission (§ 5.3).

\subsubsection{Non-iron Emission Lines}

The UV HST spectrum of I Zw 1 has a sufficiently high $\mathrm{S} / \mathrm{N}$ and spectral resolution to permit detection and identification of many weak features in addition to the strong, broad emission lines commonly observed in quasars. Table 3 lists the detected (non-iron) line features and their identification along with basic line parameters; see $\S 3.3 .2$ on line fitting for further details. L97 also identify most of these lines. We contribute with a few extra identifications and measurements: $\mathrm{Si}$ III ${ }^{\star} \lambda 1297$, and $\mathrm{Si}$ II $\lambda \lambda 1527,1533$, based on Verner et al. (1994), Morton (1991), and Wilkes (2000). We are not able to confirm the [C III] $\lambda 1907$ feature identified by L97 due to our slightly degraded resolution $(\S 2)$.

\subsubsection{Iron Emission Features}

Figure 4 shows suggested identifications of individual, mostly unblended $\mathrm{Fe}$ II and $\mathrm{Fe}$ III emission UV multiplets. ${ }^{16}$ The identifications are based on a visual inspection of clearly visible peaks in the spectrum whose positions and relative strengths were cross-correlated with the multiplet table of Moore (1950). This goes a step further than the work by L97, who mark in their Figure 2 only the expected wavelengths of some of the strongest iron multiplets. The length of the marker of a given transition in a given multiplet is proportional to its oscillator strength (the "intensity" listed by Moore is a rough measure of the relative oscillator strengths in the multiplet; note, that the physical conditions folded with the oscillator strengths will give the observed line strengths and ratios). The scaling factor is the same for all multiplets (and is arbitrarily chosen). Note that the transitions in the spectrum sometimes appear blueshifted by $1-2 \AA$ relative to the laboratory wavelengths. See e.g., Fe III UV34 at 1914 Å. Each label contains first the ionization level, then the UV multiplet number, separated by a hyphen. That is, " $2-104$ " denotes the Fe II UV104 multiplet, while " $3-158$ " denotes the Fe III UV158 multiplet, etc. Fe II and Fe III multiplets in the spectrum for which the relative transition strengths in the multiplet do not appear to follow the $\sim$ optically thin multiplet

${ }^{16}$ A full-scale version of Fig. 4 can be found at http://www.astronomy.ohio-state.edu/ vester/IronEmission. 


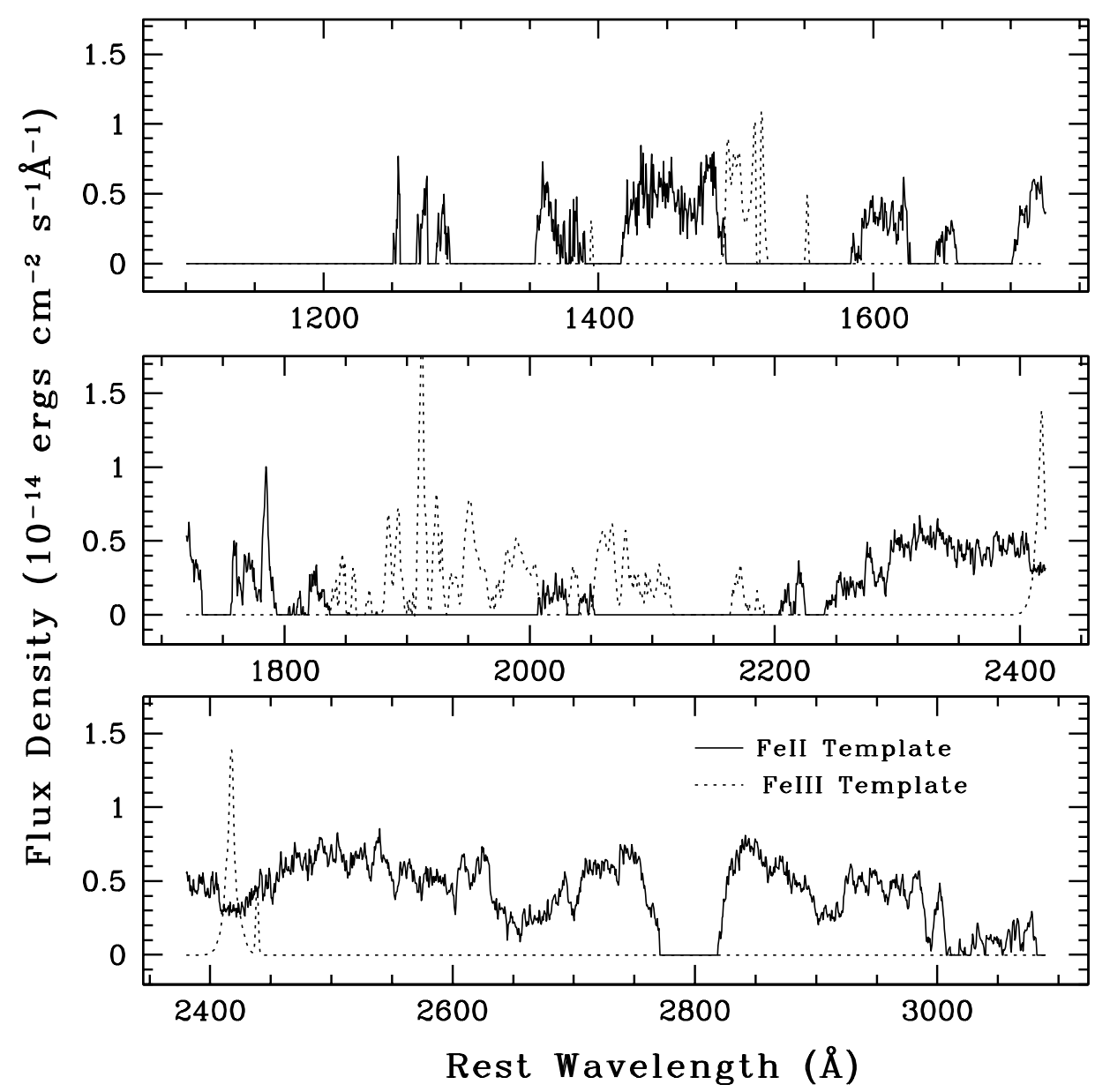

FIG. 3.-Fe III template spectrum (dotted line) overplotted on the Fe II template spectrum (solid line). Most of the Fe III features are fitted with Gaussian profiles.

strengths listed in Moore (1950) have an " $\mathrm{m}$ " attached to the labeled multiplet number. A "?" indicate that this multiplets presence is uncertain. Labels of multiplets which appear slightly blended are shown in parentheses. Square brackets denote multiplets whose presence is suggested by the fitting process (see Appendix A); Fe II UV10, which coincide with the geocoronal Ly $\alpha$ emission, is expected only. Heavily blended multiplets are not labeled, especially in the small blue bump region from $\sim 2650$ to $3090 \AA$. The lack of labeled multiplets between Ly $\alpha$ and C IV does not denote a lack of $\mathrm{Fe}$ II or $\mathrm{Fe}$ III emission transitions. Most transitions in this range are too weak (relatively) to have multiplet numbers assigned. Blueward of Ly $\alpha$ an accurate identification of iron emission multiplets requires advanced modeling of the spectrum due to the heavy blending with Ly $\alpha$ forest and other absorption lines. This is beyond the scope of this paper. L97 mark possible iron multiplets in this region. No obvious Fe II or Fe III transitions are identified immediately redward of C IV $(\sim 1550-1700 \AA)$. Marziani et al. (1996) discuss some Fe II multiplets in this wavelength region in earlier $H S T$ data of $\mathrm{I} \mathrm{Zw} 1$ (their Fig. 2). Some singlet $\mathrm{Fe}$ II and $\mathrm{Fe}$ III emission features are identified and commented on in Appendix A.

\subsubsection{Fitting Procedures}

The emission lines, listed in Tables 3 and 4, were fitted using single or multiple Gaussian components, as needed, and then subtracted from the spectrum. The Fe III and Fe II features were separated by use of the fitted Fe III lines in Table 4 to generate two templates, one for each ion (\$ 3.1). The IRAF task "splot" was used for the Gaussian fitting due to its interactive nature and the ease with which the fitted components can immediately be extracted and compared to the data. "splot" is often thought of as a tool for first order estimates but its characteristics allow us to constrain our fits faster and more conveniently than other immediately available fitting programs, due to their largely noninteractive nature.

Fitting the multiplets and line complexes was an iterative process. First the narrow components were fitted to the visible part of the lines and subtracted, then the broad components were fitted to the residuals. The broad component fits were then subtracted from the original data permitting an improved fit to be obtained for the narrow lines, which in turn were subtracted to improve the broad component fit, thereby iterating to obtain an optimal solution. Similar iterations were performed for individual (narrow) components in doublets and in regions where the narrow components are somewhat blended (e.g., C IV, Mg II, and the C III] complex). When a line complex contains two broad components (the $\mathrm{Al}$ III and $\mathrm{Mg}$ II doublet complexes, Figs. $5 d$ and $5 g$ ), they are often too heavily blended to be well separated and so were fitted by a single broad component. Two broad components were fitted simultaneously to $\mathrm{Si}$ III] and $\mathrm{C} \mathrm{III],} \mathrm{but} \mathrm{as} \mathrm{they} \mathrm{are} \mathrm{heavily} \mathrm{blended} \mathrm{the} \mathrm{fit} \mathrm{is} \mathrm{not}$ unique (see below; Fig. 5d; Appendix A). 

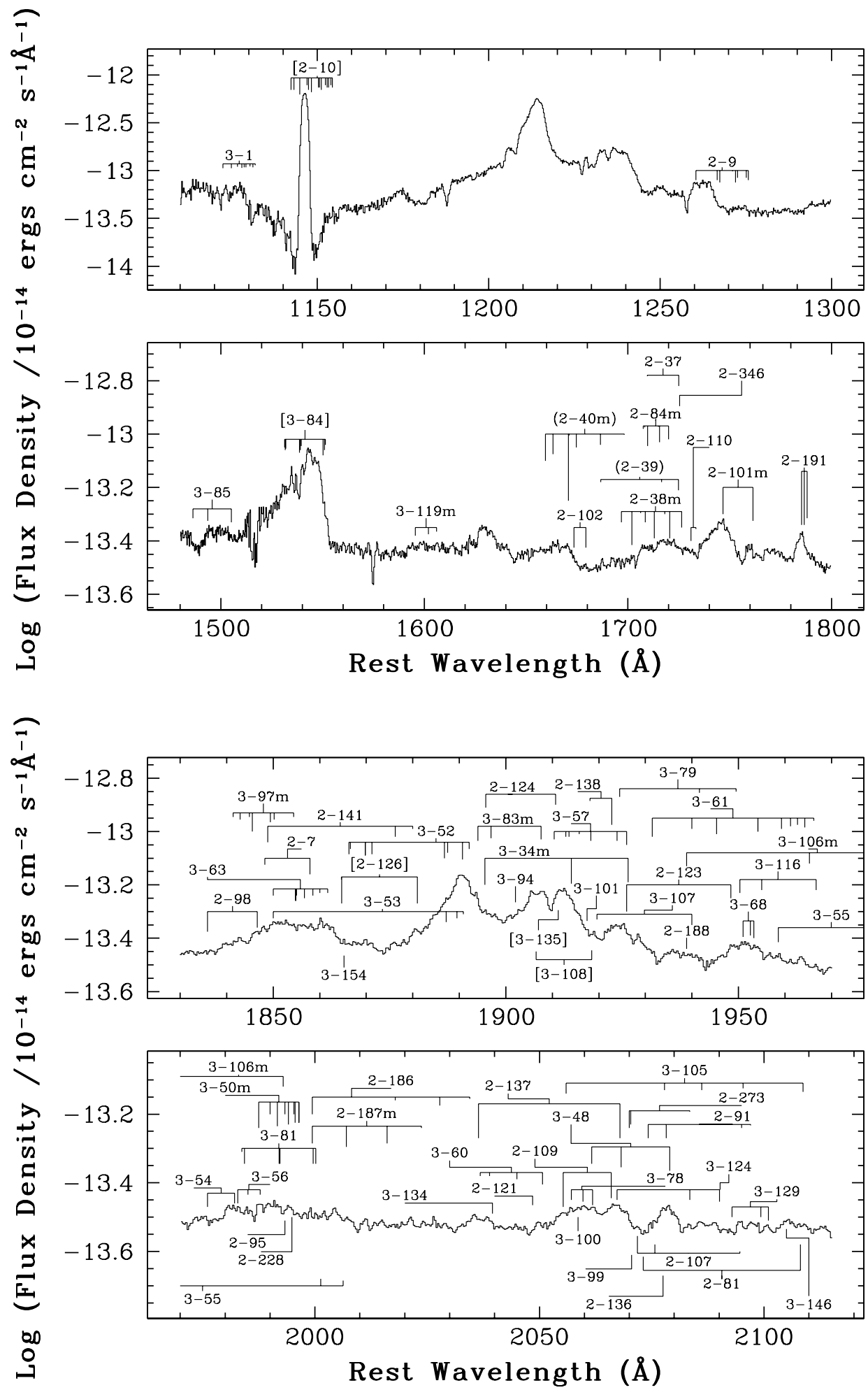

FIG. 4. - Suggested identifications of individual, mostly unblended UV Fe II and Fe III emission multiplets based on the positions and (rough) strengths listed by Moore (1950). Each label consists of the ionization level and the multiplet number separated by a hyphen. Multiplets with labels in parentheses are slightly blended. Square brackets denote multiplets which are suggested present by the fitting process (see Appendix A; " $2-10$ " is expected only). Heavily blended multiplets are not marked. Multiplets with labels ending with an " $\mathrm{m}$ " have relative transition strengths inconsistent with the optically thin laboratory strengths. Note that the positions in the spectrum of the multiplet transitions are sometimes blueshifted by $1-2 \AA$ relative to the laboratory wavelengths marked. This is particularly evident for Fe III UV34 at $1912 \AA$ (labeled “3-34 m”). See $§ 5.2$ and Appendix A regarding the relative strengths in this multiplet.

The average line width of the singlet lines is $900 \pm 150$ $\mathrm{km} \mathrm{s}^{-1}$ (Tables 3 and 4) consistent with previous studies of the line emission in I Zw 1 (Phillips 1976; BG92; van Groningen 1993; L97). However, lines with widths as small as $\sim 300-400 \mathrm{~km} \mathrm{~s}^{-1}$ (the spectral resolution limit) are detected, as discussed in $\S$ 5.1.1. These are often Fe II and Fe III lines (see Table 4).

Each Gaussian component is defined by three param- 

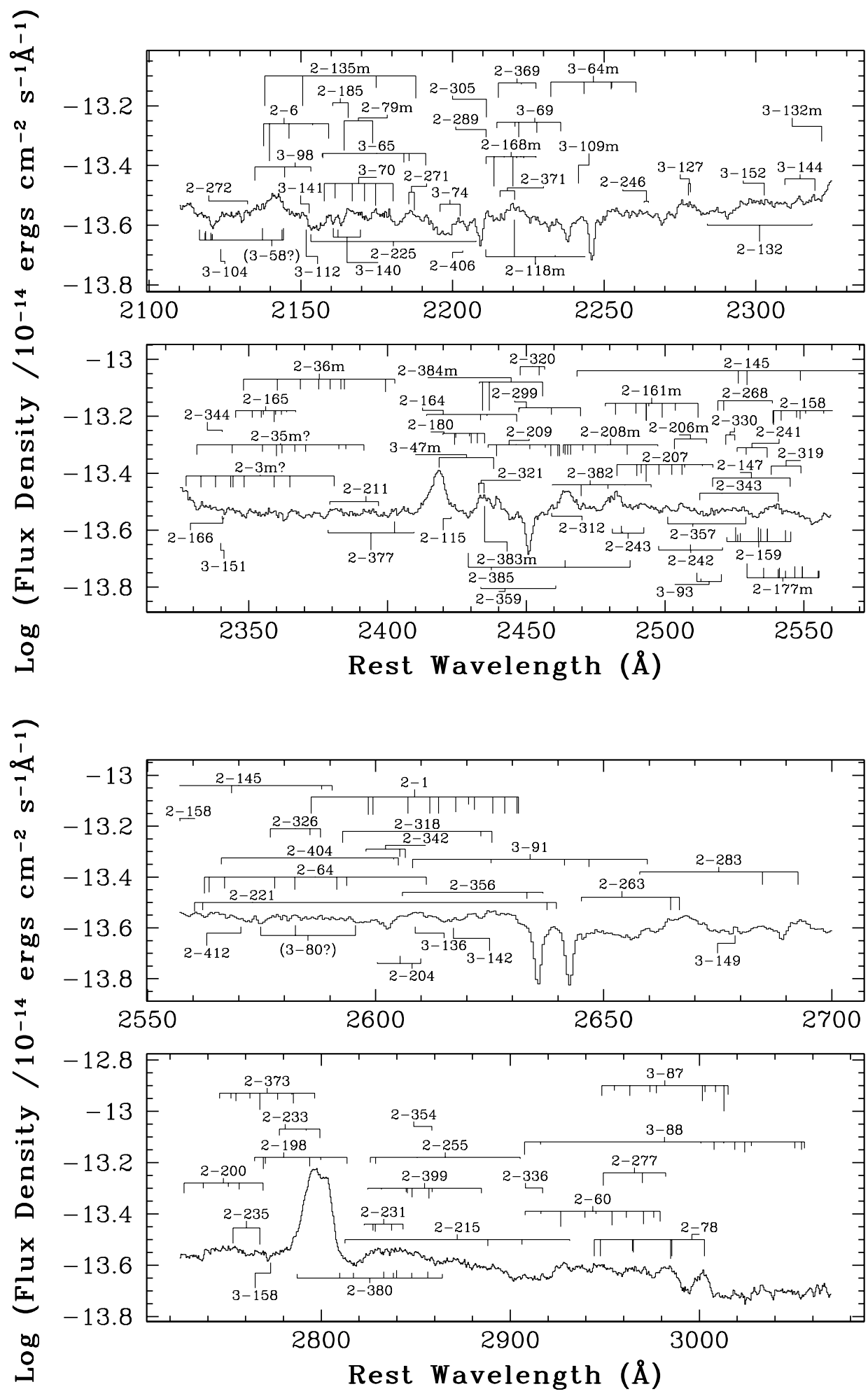

FIG. 4.-Continued

eters: position, width, and strength. In the heavily blended line complexes the absolute strengths of the individual Gaussian components are not well constrained. The uncertainty in the intensity of individual lines is estimated at $10 \%-50 \%$ depending on the width and strength of the line and on how well the data constrain the fit. The more blended the component, the more uncertain is the fit. Due to the large number of parameters involved in fitting the large line complexes, individual component solutions are not unique. In such cases, those solutions yielding parameters consistent with other lines were preferred. We note that, when the strength of any line fit was not well constrained by the observed spectrum, the affected iron residual emission was purposefully underestimated in order to avoid overcorrection of the quasar spectrum to which the template is applied. The representation of the entire line complex (i.e., the sum of all 
TABLE 4

Fitted Iron Emission FeAtures ${ }^{\mathrm{a}}$

\begin{tabular}{|c|c|c|c|c|c|c|c|c|c|}
\hline $\begin{array}{c}\text { Feature }^{b} \\
\text { (1) }\end{array}$ & $\begin{array}{c}\lambda_{\text {rest }}^{\text {meas }} \\
(\AA)^{\mathrm{c}} \\
(2)\end{array}$ & $\begin{array}{l}\lambda_{\text {lab }} \\
(\AA) \\
(3)\end{array}$ & $\begin{array}{l}\text { Ion } \\
\text { (4) }\end{array}$ & $\begin{array}{l}\text { Mult. } \\
\text { (5) }\end{array}$ & $\begin{array}{c}\Delta v^{\mathrm{d}, \mathrm{e}} \\
\left(\mathrm{km} \mathrm{s}^{-1}\right) \\
(6)\end{array}$ & $\begin{array}{l}\text { Flux }^{\mathrm{f}} \\
(7)\end{array}$ & $\begin{array}{l}\text { EW } \\
(\AA) \\
(8)\end{array}$ & $\begin{array}{c}\mathrm{FWHM}^{\mathrm{e}} \\
\left(\mathrm{km} \mathrm{s}^{-1}\right) \\
(9)\end{array}$ & $\begin{array}{l}\text { Comments } \\
\text { (10) }\end{array}$ \\
\hline \multirow[t]{3}{*}{$\lambda 1495$. } & 1493.2 & $1493.6^{\mathrm{g}}$ & $\mathrm{Fe}$ III & UV85 & -80 & $\ldots$ & $\ldots$ & & \\
\hline & 1494.1 & 1495.2 & $\mathrm{Fe}$ III & - & -220 & 3.40 & 1.0 & 710 & \\
\hline & 1498.6 & 1498.8 & $\mathrm{Fe}$ III & - & -40 & 2.87 & 0.8 & 710 & \\
\hline \multirow[t]{3}{*}{$\lambda 1505 \ldots \ldots \ldots$} & 1502.2 & 1503.0 & $\mathrm{Fe}$ III & - & -160 & 2.59 & 0.8 & 678 & \\
\hline & 1505.4 & 1505.2 & $\mathrm{Fe}$ III & UV85 & +40 & 1.10 & 0.3 & 678 & \\
\hline & 1509.2 & 1509.2 & $\mathrm{Fe}$ III & - & 0 & 1.20 & 0.3 & 678 & \\
\hline \multirow{2}{*}{$\lambda 1515 \ldots \ldots \ldots$} & 1513.9 & 1514.5 & Fe III & - & -120 & 4.85 & 1.4 & 900 & \\
\hline & 1518.5 & 1518.8 & $\mathrm{Fe}$ III & - & -60 & 5.00 & 1.5 & 836 & \\
\hline \multirow[t]{3}{*}{$\lambda 1520$. } & 1521.2 & 1524.5 & $\mathrm{Fe} \mathrm{III}^{\mathrm{h}}$ & - & -650 & 1.73 & 0.5 & 478 & Iron or $\mathrm{Si}$ III? \\
\hline & 1524.0 & 1525.0 & $\mathrm{Fe} \mathrm{III}^{\mathrm{h}}$ & - & -195 & 0.86 & 0.3 & 478 & \\
\hline & & 1525.8 & $\mathrm{Fe}$ III & - & -355 & $\ldots$ & $\ldots$ & $\ldots$ & Alt. ID \\
\hline$\lambda 1786_{\text {total }} \ldots \ldots$ & 1785.4 & 1786.0 & $\mathrm{Fe}$ II & UV191 & -100 & $\ldots$ & $\ldots$ & 550 & Narrow, 1 Gaussian \\
\hline \multirow[t]{3}{*}{$\lambda 1786_{\text {total }} \cdots \cdots$} & 1783.0 & 1785.3 & $\mathrm{Fe}$ II & UV191 & -385 & 1.53 & 0.5 & 445 & 3 Gauss model Ref. (4) \\
\hline & 1785.6 & 1786.7 & $\mathrm{Fe}$ II & UV191 & -185 & 2.63 & 0.8 & 445 & Ditto \\
\hline & 1788.6 & 1788.0 & $\mathrm{Fe}$ II & UV191 & +100 & 0.85 & 0.3 & 445 & Ditto \\
\hline \multirow[t]{2}{*}{$\lambda 1850 \ldots \ldots \ldots$} & 1850.3 & 1850.0 & & UV53,63 & +50 & $\ldots$ & $\ldots$ & $\ldots$ & Possibly blended with UV97 \\
\hline & & $1849.8^{\mathrm{i}}$ & $\mathrm{Fe}$ III & UV97 & +80 & 0.45 & 0.1 & 265 & $\lambda 1849.4,1850.2$ \\
\hline \multirow{3}{*}{$\lambda 1855 \ldots \ldots \ldots$} & 1856.2 & $1854.9^{\mathrm{i}}$ & $\mathrm{Fe}$ III & UV63 & +210 & 0.41 & 0.1 & 295 & $\lambda 1854.8,1855.0$ \\
\hline & 1857.0 & 1856.7 & Fe III & UV63 & +50 & 0.29 & 0.1 & 265 & \\
\hline & 1858.2 & 1858.5 & $\mathrm{Fe}$ III & UV63 & -50 & 0.24 & 0.1 & 140 & \\
\hline \multirow{6}{*}{$\lambda 1870 \ldots \ldots \ldots$} & 1867.9 & $1866.5^{\mathrm{i}}$ & $\mathrm{Fe}$ III & UV52 & +225 & 0.20 & 0.1 & 260 & $\lambda \lambda 1866.3,1866.6$ \\
\hline & & $1867.6^{\mathrm{i}}$ & $\mathrm{Fe}$ II & - & +50 & $\ldots$ & $\ldots$ & $\ldots$ & $\lambda \lambda 1867.2,1867.9$ Alt. ID \\
\hline & 1869.7 & 1869.8 & $\mathrm{Fe}$ III & UV52 & -15 & 0.22 & 0.1 & 140 & \\
\hline & & 1869.6 & $\mathrm{Fe}$ II & - & +15 & $\ldots$ & $\ldots$ & $\ldots$ & Alt.ID \\
\hline & 1871.0 & 1871.2 & $\mathrm{Fe}$ III & UV52 & -30 & 0.09 & 0.03 & 95 & \\
\hline & & 1871.0 & $\mathrm{Fe}$ II & - & 0 & $\ldots$ & $\ldots$ & $\ldots$ & Alt. ID \\
\hline \multirow[t]{3}{*}{$\lambda 1885 \ldots \ldots \ldots$} & 1884.6 & $1885.6^{\mathrm{i}}$ & $\mathrm{Fe}$ III & UV96 & -160 & 1.808 & 0.6 & 640 & $\lambda \lambda 1885.1,1886.0$ \\
\hline & 1886.0 & 1886.8 & $\mathrm{Fe}$ III & UV52 & -125 & 1.43 & 0.5 & 640 & \\
\hline & 1887.1 & $1887.5^{\mathrm{g}}$ & $\mathrm{Fe}$ III & $\mathrm{UV} 52,{ }^{\mathrm{j}} 53^{\mathrm{j}}$ & -65 & & & & \\
\hline \multirow[t]{6}{*}{$\lambda 1895 \ldots \ldots \ldots$} & 1892.7 & 1894.8 & $\mathrm{Fe}$ III & UV34 & -330 & 3.76 & 1.2 & Model B & Ref. (3) \\
\hline & & 1895.5 & Fe III & UV34 & -445 & $\ldots$ & $\ldots$ & $\ldots$ & Ref. $(\mathrm{m})$ \\
\hline & & $1894.0^{\mathrm{i}}$ & $\mathrm{Fe} \mathrm{III}^{\mathrm{h}}$ & UV96,83 & -205 & $\cdots$ & $\cdots$ & $\ldots$ & $\lambda \lambda 1892.9,1895.0(96), 1894.0(83)$ with Si III 1892 + UV96,83? \\
\hline & 1896.6 & $1897.1^{\mathrm{i}}$ & Fe III & UV83 & -80 & 0.60 & 0.2 & 310 & $\lambda \lambda 1896.8,1897.4$ (Kurucz) \\
\hline & & 1896.8 & & UV83 & -30 & & & & (Moore) \\
\hline & 1901.0 & $1901.1^{\mathrm{g}}$ & $\mathrm{Fe}$ III & UV95 & -15 & & & & \\
\hline \multirow[t]{8}{*}{$\lambda 1912 \ldots \ldots \ldots$} & 1912.0 & 1914.1 & Fe III & UV34 & -330 & 10.04 & 3.3 & Model B & See also Appendix A, Ref. (4) \\
\hline & & 1911.3 & $\mathrm{Fe}$ III & UV135 & +110 & $\ldots$ & $\ldots$ & $\ldots$ & Possibly blended with UV34,57 \\
\hline & & 1912.9 & Fe III & UV57 & -140 & $\ldots$ & $\ldots$ & $\ldots$ & Weak, blended with UV34,135? \\
\hline & & 1915.1 & Fe III & UV51 & -485 & $\ldots$ & $\ldots$ & $\ldots$ & Alt.ID, Ref. (5) \\
\hline & & $1916.5^{\mathrm{g}}$ & $\mathrm{Fe}$ III & UV95 & $\ldots$ & $\ldots$ & $\ldots$ & $\ldots$ & Blended \\
\hline & & 1917.5 & $\mathrm{Fe} \mathrm{III}^{\mathrm{h}, \mathbf{k}}$ & UV101 & -860 & $\ldots$ & $\ldots$ & $\ldots$ & Alt.ID \\
\hline & 1917.6 & $1917.4^{\mathrm{g}}$ & $\mathrm{Fe}$ III & UV95 & +30 & $\ldots$ & $\ldots$ & $\ldots$ & \\
\hline & 1922.7 & $1923.0^{\mathrm{g}}$ & $\mathrm{Fe}$ III & UV95 & -45 & $\ldots$ & $\ldots$ & $\ldots$ & \\
\hline \multirow[t]{7}{*}{$\lambda 1924 \ldots \ldots \ldots$} & 1923.6 & $1923.5^{\mathrm{i}}$ & $\mathrm{Fe}$ III & UV95,57 & +15 & 1.49 & 0.5 & 675 & $\lambda \lambda 1923.0(95), 1923.9(57)$ \\
\hline & 1924.5 & 1924.5 & $\mathrm{Fe}$ III & UV79 & 0 & 3.36 & 1.1 & 750 & \\
\hline & 1923.9 & 1926.3 & $\mathrm{Fe}$ III & UV34 & -375 & 4.24 & 1.4 & Model B & \\
\hline & 1925.5 & 1926.0 & $\mathrm{Fe}$ II & UV123 & -75 & & & & Blended with UV34,57? \\
\hline & & $1926.0^{\mathrm{g}}$ & $\mathrm{Fe}$ III & UV57 & $\ldots$ & $\ldots$ & $\ldots$ & $\ldots$ & Blended \\
\hline & & $1928.3^{\mathrm{g}}$ & $\mathrm{Fe}$ III & UV95 & $\ldots$ & $\ldots$ & $\ldots$ & $\ldots$ & Blended \\
\hline & 1932.8 & $1932.8^{\mathrm{g}}$ & $\mathrm{Fe}$ III & UV95 & 0 & $\ldots$ & $\ldots$ & $\ldots$ & \\
\hline \multirow[t]{2}{*}{$\lambda 1935 \ldots \ldots \ldots$} & 1935.9 & 1935.3 & $\mathrm{Fe}$ II & UV96 & -90 & 0.76 & 0.3 & 385 & UV96: $1936.8,{ }^{\mathrm{g}} 1917.3$ blended \\
\hline & 1938.7 & 1938.8 & $\mathrm{Fe}$ III & UV95 & -15 & 0.60 & 0.2 & 385 & \\
\hline
\end{tabular}


TABLE 4 -Continued

\begin{tabular}{|c|c|c|c|c|c|c|c|c|c|}
\hline $\begin{array}{c}\text { Feature }^{b} \\
\text { (1) }\end{array}$ & $\begin{array}{c}\lambda_{\text {rest }}^{\text {meas }} \\
(\AA)^{\mathrm{c}} \\
(2)\end{array}$ & $\begin{array}{l}\lambda_{\text {lab }} \\
(\AA) \\
(3)\end{array}$ & $\begin{array}{l}\text { Ion } \\
(4)\end{array}$ & $\begin{array}{l}\text { Mult. } \\
\text { (5) }\end{array}$ & $\begin{array}{c}\Delta v^{\mathrm{d}, \mathrm{e}} \\
\left(\mathrm{km} \mathrm{s}^{-1}\right) \\
(6)\end{array}$ & $\begin{array}{l}\text { Flux }^{\mathrm{f}} \\
(7)\end{array}$ & $\begin{array}{l}\text { EW } \\
(\AA) \\
(8)\end{array}$ & $\begin{array}{c}\text { FWHM }^{\mathrm{e}} \\
\left(\mathrm{km} \mathrm{s}^{-1}\right) \\
(9)\end{array}$ & $\begin{array}{c}\text { Comments } \\
\text { (10) }\end{array}$ \\
\hline & 1941.0 & $1941.1^{\mathrm{i}}$ & $\mathrm{Fe}$ III & UV79 & -15 & 0.59 & 0.2 & 385 & $\lambda \lambda 1940.6,1941.6$ \\
\hline \multirow[t]{10}{*}{$\lambda 1950 \ldots \ldots$} & 1948.0 & 1948.4 & $\mathrm{Fe}$ II & UV123 & -60 & 2.40 & 0.8 & 830 & \\
\hline & 1949.7 & $1949.7^{\mathrm{g}}$ & Fe III & UV95 & 0 & $\ldots$ & $\ldots$ & $\ldots$ & \\
\hline & 1951.6 & 1951.3 & $\mathrm{Fe}$ III & UV68 & +45 & 2.39 & 0.8 & 825 & Ref. (m): $\lambda 1951.0, \Delta v=+90$ \\
\hline & 1953.1 & $1953.0^{\mathrm{i}}$ & Fe III & UV68 & +15 & 1.70 & 0.6 & 825 & $\lambda \lambda 1952.6,1953.3$ \\
\hline & & 1953.3 & Fe III & UV82 & -30 & $\ldots$ & $\ldots$ & $\ldots$ & Blended with UV68? \\
\hline & 1953.5 & $1953.5^{i, 1}$ & Fe III & UV82 & 0 & $\ldots$ & $\ldots$ & $\ldots$ & \\
\hline & 1957.9 & 1957.9 & $\mathrm{Fe}$ III & UV147 & 0 & 2.04 & 0.7 & 825 & \\
\hline & 1958.8 & $1958.6^{\mathrm{g}}$ & Fe III & UV55 & +30 & $\ldots$ & $\ldots$ & $\ldots$ & \\
\hline & & $1960.3^{\mathrm{g}}$ & Fe III & UV82 & $\ldots$ & $\ldots$ & $\ldots$ & $\ldots$ & Blended \\
\hline & 1963.6 & $1964.3^{\mathrm{i}}$ & Fe III & UV82 & -100 & 1.67 & 0.6 & 820 & $\lambda \lambda 1964.0,1964.2,1964.8$ \\
\hline \multirow[t]{2}{*}{$\lambda 1975 \ldots \ldots$} & 1973.5 & 1976.1 & $\mathrm{Fe}$ III $^{\mathrm{h}}$ & UV54 & -395 & 0.35 & 0.1 & 255 & ID? \\
\hline & 1975.4 & 1976.1 & Fe III & UV54 & -105 & 0.42 & 0.1 & 255 & \\
\hline \multirow[t]{12}{*}{$\lambda 1990 \ldots \ldots$} & 1977.5 & $1977.3^{1}$ & $\ldots$ & $\ldots$ & +30 & 0.14 & 0.1 & 190 & \\
\hline & 1981.5 & $1982.5^{\mathrm{i}}$ & $\mathrm{Fe}$ III & UV54,56 & -150 & 2.17 & 0.7 & 735 & $\lambda \lambda 1982.1(54), 1982.8(56)$ \\
\hline & 1985.5 & $1985.0^{\mathrm{i}}$ & $\mathrm{Fe}$ III & UV56,81 & +80 & 1.00 & 0.3 & 730 & $\lambda \lambda 1985.1,1987.8(57), 1984.3(81)$ \\
\hline & 1989.6 & $1989.5^{\mathrm{i}}$ & Fe III & UV50 & +15 & 2.41 & 0.8 & 730 & $\lambda \lambda 1987.5,1990.0,1991.6$ \\
\hline & 1994.2 & $1994.2^{\mathrm{i}}$ & $\mathrm{Fe}$ III & UV50 & 0 & 1.85 & 0.6 & 730 & $\lambda \lambda 1993.3,1994.1,1995.3$ \\
\hline & 1994.2 & $1992.1^{\mathrm{i}}$ & Fe III & UV81 & +300 & 1.85 & 0.6 & 730 & $\lambda \lambda 1992.0,1992.2$ \\
\hline & 1999.2 & $1999.8^{\mathrm{i}}$ & Fe III & UV55,81 & -90 & 1.48 & 0.5 & 725 & $\lambda \lambda 1999.6(55,81), 2000.2(81)$ \\
\hline & & 1999.4 & $\mathrm{Fe}$ II & UV186 & -30 & $\ldots$ & $\ldots$ & $\ldots$ & Blended with UV55,81? \\
\hline & 2004.5 & 2004.1 & $\mathrm{Fe}$ II & UV83 & +60 & 1.79 & 0.6 & 725 & \\
\hline & & 2003.5 & Fe III & UV55 & +150 & $\ldots$ & $\ldots$ & $\ldots$ & Blended with UV83? \\
\hline & 2007.2 & $2007.8^{\mathrm{g}}$ & Fe III & UV55 & -90 & $\ldots$ & $\ldots$ & $\ldots$ & \\
\hline & & $2017.9^{\mathrm{g}}$ & $\mathrm{Fe}$ II & UV186 & $\ldots$ & $\ldots$ & $\ldots$ & $\ldots$ & Blended \\
\hline$\lambda 2030 \ldots \ldots$ & 2032.5 & 2033.1 & $\cdots$ & $\cdots$ & -90 & 0.22 & 0.1 & 255 & ID? \\
\hline \multirow[t]{3}{*}{$\lambda 2035 \ldots \ldots$} & 2035.3 & 2035.1 & $\mathrm{Fe}$ II & UV186 & +30 & 0.97 & 0.3 & 410 & (Kurucz) \\
\hline & & 2034.5 & & & +115 & $\ldots$ & $\ldots$ & $\ldots$ & (Moore) \\
\hline & 2036.6 & $2036.8^{\mathrm{g}}$ & $\mathrm{Fe}$ III & UV60 & -30 & $\cdots$ & $\cdots$ & $\cdots$ & \\
\hline \multirow[t]{4}{*}{$\lambda 2040 \ldots \ldots$} & 2038.5 & 2038.9 & $\mathrm{Fe}$ III & UV60 & -60 & 0.83 & 0.3 & 410 & \\
\hline & & 2039.5 & Fe III & UV134 & -145 & $\ldots$ & $\ldots$ & $\ldots$ & Alt. ID \\
\hline & 2044.7 & $2045.0^{\mathrm{g}}$ & $\mathrm{Fe}$ III & UV60 & -45 & $\ldots$ & $\ldots$ & $\ldots$ & \\
\hline & 2049.8 & $2050.7^{\mathrm{g}}$ & Fe III & UV60 & -130 & $\cdots$ & $\cdots$ & $\cdots$ & \\
\hline \multirow[t]{8}{*}{$\lambda 2060 \ldots \ldots$} & 2055.6 & 2056.8 & $\mathrm{Fe}$ III & $\mathrm{UV} 105^{\mathrm{m}}$ & -175 & 2.25 & 0.8 & 815 & Ref. (m): $\lambda 2055.9, \Delta v=-45$ \\
\hline & 2057.1 & $2056.9^{\mathrm{g}}$ & Fe III & UV78 & +30 & $\ldots$ & $\ldots$ & $\ldots$ & \\
\hline & 2059.7 & $2059.7^{\mathrm{g}}$ & $\mathrm{Fe}$ III & UV78 & 0 & $\ldots$ & $\ldots$ & $\ldots$ & \\
\hline & 2060.2 & 2058.6 & Fe III & UV100 & +160 & 1.98 & 0.7 & 810 & Blended with UV78 $\lambda 2059.7 ?$ \\
\hline & 2061.3 & 2061.6 & $\mathrm{Fe}$ III & UV48 & -45 & 1.18 & 0.4 & 805 & Blended with UV78 $\lambda 2061.8 ?$ \\
\hline & 2061.8 & $2061.8^{\mathrm{g}}$ & $\mathrm{Fe}$ III & UV78 & 0 & $\ldots$ & $\ldots$ & $\ldots$ & \\
\hline & 2067.2 & 2068.2 & Fe III & UV48 & -145 & 3.59 & 1.3 & 810 & \\
\hline & 2077.6 & $2077.8^{\mathrm{g}}$ & $\mathrm{Fe}$ III & UV105 & -30 & $\ldots$ & $\ldots$ & $\ldots$ & \\
\hline$\lambda 2080 \ldots \ldots$ & 2078.3 & 2079.0 & $\mathrm{Fe}$ III & UV48 & -100 & 3.11 & 1.1 & 735 & Ref. (2) \\
\hline \multirow[t]{10}{*}{$\lambda 2100 \ldots \ldots$} & 2083.0 & 2083.1 & $\mathrm{Fe}$ III & - & -15 & 0.42 & 0.2 & 280 & \\
\hline & & 2083.5 & $\mathrm{Fe}$ III $^{h}$ & UV124 & -70 & 0.42 & 0.2 & 280 & Alt. ID \\
\hline & 2085.9 & 2085.0 & Fe III & UV67 & +130 & 0.79 & 0.3 & 375 & \\
\hline & 2086.0 & $2086.1^{\mathrm{g}}$ & Fe III & UV105 & -15 & $\ldots$ & $\ldots$ & $\ldots$ & \\
\hline & 2088.8 & $2088.7^{\mathrm{i}}$ & $\mathrm{Fe}$ III & UV67,77 & +15 & 0.62 & 0.2 & 320 & $\lambda \lambda 2087.8,2088.6,2089.8(77), 2088.6(67)$ \\
\hline & & $2088.1^{\mathrm{i}}$ & & & +100 & & & & Ref. (m): ג2087.1,2087.9,2089.1(77) \\
\hline & 2091.7 & 2092.0 & $\mathrm{Fe}$ III & UV77 & -45 & 0.31 & 0.1 & 305 & Ref. (m): $\lambda 2091.3, \Delta v=+60$ \\
\hline & 2094.7 & 2094.2 & Fe III & UV77 & +70 & 0.57 & 0.2 & 255 & Ref. (m): $\lambda 2093.5, \Delta v=+170$ \\
\hline & 2095.0 & $2095.3^{\mathrm{g}}$ & Fe III & UV105 & -40 & $\ldots$ & $\ldots$ & $\ldots$ & \\
\hline & 2097.9 & $2098.3^{\mathrm{i}}$ & Fe III & UV67,66 & -60 & 0.76 & 0.3 & 400 & $\lambda \lambda 2098.2,2098.4$ \\
\hline
\end{tabular}


TABLE 4-Continued

\begin{tabular}{|c|c|c|c|c|c|c|c|c|c|}
\hline $\begin{array}{c}\text { Feature }^{b} \\
\text { (1) }\end{array}$ & $\begin{array}{c}\lambda_{\text {rest }}^{\text {meas }} \\
(\AA)^{\mathrm{c}} \\
(2)\end{array}$ & $\begin{array}{l}\lambda_{\text {lab }} \\
(\AA) \\
(3)\end{array}$ & $\begin{array}{l}\text { Ion } \\
\text { (4) }\end{array}$ & $\begin{array}{l}\text { Mult. } \\
\text { (5) }\end{array}$ & $\begin{array}{c}\Delta v^{\mathrm{d}, \mathrm{e}} \\
\left(\mathrm{km} \mathrm{s}^{-1}\right) \\
(6)\end{array}$ & $\begin{array}{c}\text { Flux }^{\mathrm{f}} \\
\text { (7) }\end{array}$ & $\begin{array}{l}\mathrm{EW} \\
(\AA) \\
(8)\end{array}$ & $\begin{array}{c}\mathrm{FWHM}^{\mathrm{e}} \\
\left(\mathrm{km} \mathrm{s}^{-1}\right) \\
(9)\end{array}$ & $\begin{array}{c}\text { Comments } \\
\text { (10) }\end{array}$ \\
\hline & 2103.4 & 2103.8 & $\mathrm{Fe}$ III & UV $66^{\mathrm{j}}$ & -60 & 0.47 & 0.2 & 380 & \\
\hline & 2105.8 & 2105.0 & $\mathrm{Fe}$ III & UV146 & +115 & 0.85 & 0.3 & 350 & \\
\hline & & 2107.3 & $\mathrm{Fe}$ III & UV66 & -215 & $\ldots$ & $\ldots$ & $\ldots$ & Blended with UV146? \\
\hline & 2108.4 & 2108.7 & $\mathrm{Fe}$ III & UV105 & -40 & 0.32 & 0.1 & 340 & \\
\hline & & 2107.6 & $\mathrm{Fe}$ II & UV250 & +115 & $\ldots$ & $\ldots$ & $\ldots$ & Blended with Fe III UV105? \\
\hline & 2109.6 & 2109.4 & $\mathrm{Fe}$ III & UV105 & +30 & 0.36 & 0.1 & 375 & \\
\hline & 2113.2 & $2114.3^{\mathrm{i}}$ & $\mathrm{Fe}$ III $^{\mathrm{h}}$ & - & -155 & 0.90 & 0.3 & 465 & $\lambda \lambda 2114.0,2114.6$ \\
\hline$\lambda 2418 \ldots \ldots \ldots$ & 2418.4 & 2418.6 & Fe III & UV47 & -25 & $\ldots$ & $\ldots$ & 715 & Narrow, 1 Gauss \\
\hline$\lambda 2418_{\text {total }} \ldots \ldots$ & & 2418.6 & $\mathrm{Fe}$ III & UV47 & $\ldots$ & 12.27 & 5.1 & $\ldots$ & 3 Gauss fit, Ref. (1) \\
\hline \multirow[t]{3}{*}{$\lambda 2418 \ldots \ldots \ldots$} & 2415.0 & 2418.6 & $\mathrm{Fe}$ III & UV47 & -445 & 1.20 & 0.5 & 590 & Narrow \\
\hline & 2418.6 & 2418.6 & $\mathrm{Fe}$ III & UV47 & 0 & 3.61 & 1.5 & 475 & Narrow \\
\hline & 2418.8 & 2418.6 & $\mathrm{Fe}$ III & UV47 & +25 & 7.46 & 3.1 & 1910 & Broad \\
\hline$\lambda 2438 \ldots \ldots \ldots$ & 2439.6 & 2438.9 & $\mathrm{Fe}$ III & UV47 & +85 & 1.17 & 0.5 & 300 & Ref. (m): $\lambda 2438.2, \Delta v=+170$ \\
\hline
\end{tabular}

${ }^{\text {a }}$ The features listed here are those which are strong enough to be fitted and for which fitting was required in the deblending process of the non-iron lines. See also Appendix A for details on each fitted feature.

${ }^{\mathrm{b}}$ A simultaneous fit was made to each line blend listed as a Feature (col. [1]) unless otherwise noted.

${ }^{c}$ If no wavelength is listed the entry is an alternative identification to the entry above it.

d $\Delta v$ is the line peak velocity shift from the expected rest frame position.

e The resolution is $470 \mathrm{~km} \mathrm{~s}^{-1}$ at $\lambda 1200 \AA, 355 \mathrm{~km} \mathrm{~s}^{-1}$ at $1600 \AA, 280 \mathrm{~km} \mathrm{~s}^{-1}$ at $2000 \AA, 240 \mathrm{~km} \mathrm{~s}^{-1}$ at $2400 \AA$ and $200 \mathrm{~km} \mathrm{~s}^{-1}$ at $2800 \AA$ (Leitherer 1995).

${ }^{\mathrm{f}}$ Units of $10^{-14} \mathrm{ergs} \mathrm{cm}^{-2} \mathrm{~s}^{-1}$.

${ }^{g}$ The transition is detected only and/or is likely present; feature is not modeled and measured.

$\mathrm{h}$ The line identification is uncertain.

${ }^{i}$ Multiplet wavelength or mean wavelength of more than one transition. The wavelengths are listed in the "Comments" column.

${ }^{j}$ Other transitions of this multiplet are possibly present, but (heavily) blended.

k This transition may also contribute, but it is likely much weaker than the transition strength indicate.

${ }^{1}$ A separate fit was made to this component; nonsimultaneous from the other transitions.

${ }^{\mathrm{m}}$ Transitions without multiplet number contribute as well.

REFERENCES.-Moore 1950 (m); Kurucz 1997 (air-wavelength for $\lambda>2000 \AA ̊$ ), unless otherwise noted. (1) Ekberg 1993; (2) Hartig \& Baldwin 1986; (3) Marziani et al. 1996; (4) Penston et al. 1983; (5) Fuhr et al. 1988.

the individual components) was, however, always well constrained by the data, and the estimated errors are of order a few percent.

Examples of the emission-line fits can be found in Figure 5. The individual measurements of fitted non-iron and iron lines are listed in Tables 3 and 4, respectively. Measurements are based on the individual Gaussian components making up the line profile; sums for the line complexes are also listed. The equivalent widths are always measured relative to the adopted (global) continuum level (§ 2). Very weak iron features were not fitted.

We emphasize that the multiple Gaussian component fitting is simply a tool, and we make no assumptions as to the mechanism responsible for the overall profile shapes. Thus, as no physical meaning is associated with the individual Gaussian component fits, the absolute and relative fluxes of the fitted components cannot be interpreted in terms of physically distinct emitting regions. Extreme care should always be exercised when interpreting the measurements, particularly of weak features. Features for which certain fitting parameters and/or the line identifications are particularly uncertain are mentioned in Appendix A.

\subsection{Comparison with Earlier UV Iron Templates and Models}

Despite the wealth of work on modeling the iron emission in quasars there is no UV template electronically available which can be applied to observed quasar spectra, including our own (M. Vestergaard et al. 2001, in preparation), for removal of the UV Fe II emission. One other empirical UV template is available in the literature: the Corbin \& Boroson (1996) template covering the iron bump around $\mathrm{Mg}$ II. Below we briefly comment on how this compares to our template in the overlapping region. A couple of synthetic Fe II spectra have been generated based on the available knowledge of the iron emission mechanism, the quality of the atomic data, and the computing facilities at the time (Wills et al. 1985; Verner et al. 1999). We briefly discuss the apparent differences with the current iron template.

\subsubsection{Empirical UV Templates}

Corbin \& Boroson (1996) present a $2300-3000 \AA$ iron template also derived from the $H S T$ spectrum of I $\mathrm{Zw} 1$ (L97). Although a detailed comparison is not possible (the digital spectrum is not available and Fig. 2 in Corbin \& Boroson 1996 shows an already broadened version of the template), it is clear that differences are present due to their use of interpolation rather than deblending to remove noniron features. This is particularly clear around $\mathrm{Mg}$ II where interpolation across the $\mathrm{Mg}$ II line likely overestimates the iron emission strength by $\sim 300 \%$; the Fe II emission level is at most $15 \%-20 \%$ of the peak height of the $\mathrm{Fe}$ II on either side of the Mg II gap (cf. Fig. 12 in Verner et al. 1999). In contrast, our approach (§3.3.2) is to purposefully underestimate the iron emission when the strength of the iron and/or 

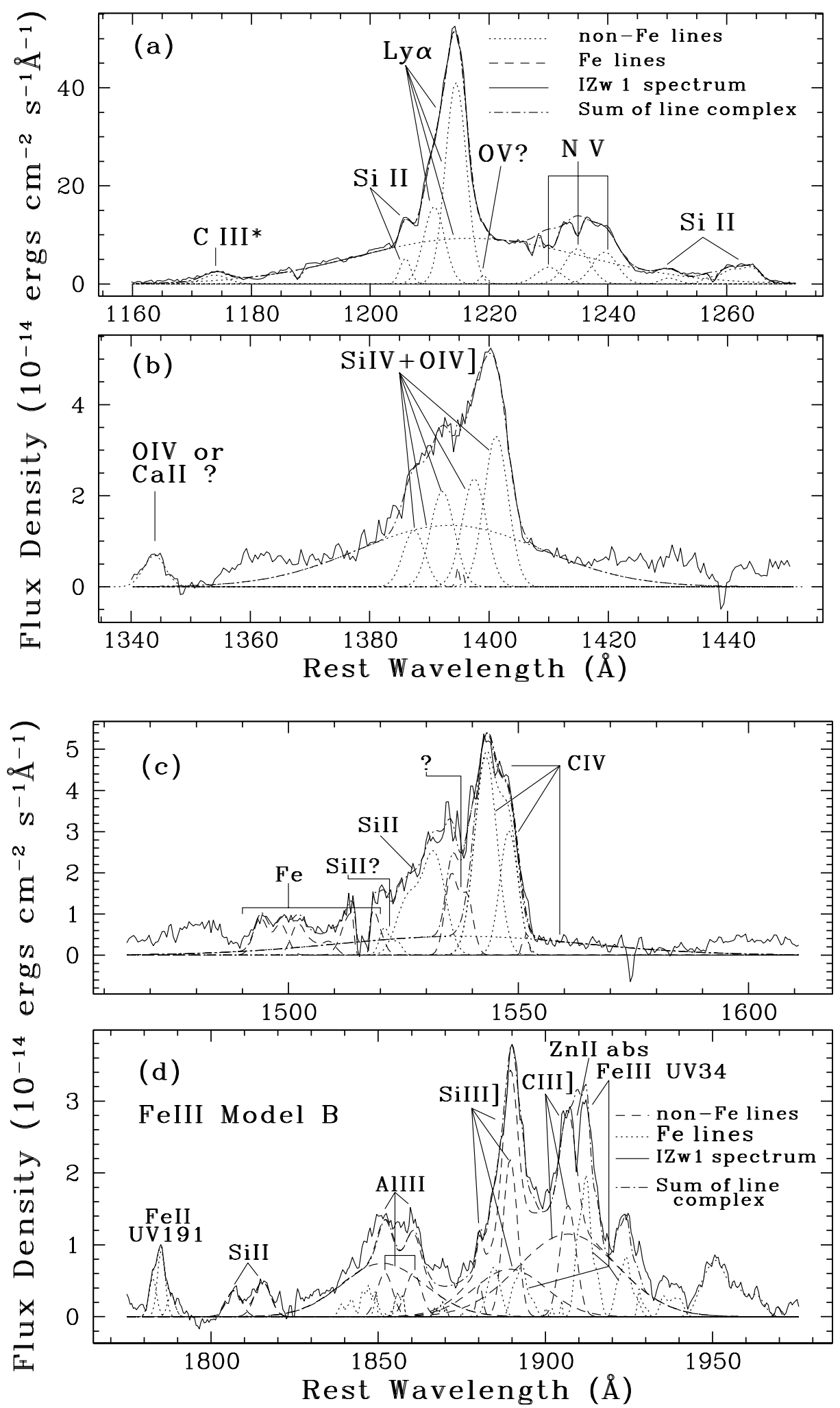

FIG. 5.-Examples of line fitting. Each individual component is shown as well as their sum (dot-dashed line) superposed on the data (solid line). The non-iron emission is dotted while the fitted iron emission is dashed. Note, the fit is sometimes so good it entirely coincides with the spectrum. (a) Ly $\alpha$ complex; note the strongly blue-asymmetric $\mathrm{N} v$ profile. This is also seen in the nondeblended $\mathrm{C}$ IV and $\mathrm{Mg}$ II profiles (Appendix A). (b) Si IV $+\mathrm{O}$ IV] $\lambda 1400$ blend. (c) C IV complex; “?”: the identification of this feature is uncertain; see text. (d) Fe II UV191, and C III] complex [Note, the Zn II ISM absorption at $1910 \AA$. Both the sum of the non-iron emission line fits and the sum of non-iron and iron fits are shown in dot-dashed curves. The latter (full) sum coincides so well with the data in many places that it is not easily seen, e.g., around Si III], C III], and Fe III redward of C III]. The fit to Fe II UV191 also coincides well with the data]. The fit to Fe III UV34 is model B. (e) Other sample model fits to Fe III UV34 as discussed in text. The individual fits to Si III] and C III] are not shown, but the residuals are. $(f)$ Fe III UV47 22418,2438 , and the non-iron features nearby. $(g) \mathrm{Mg}$ II. 

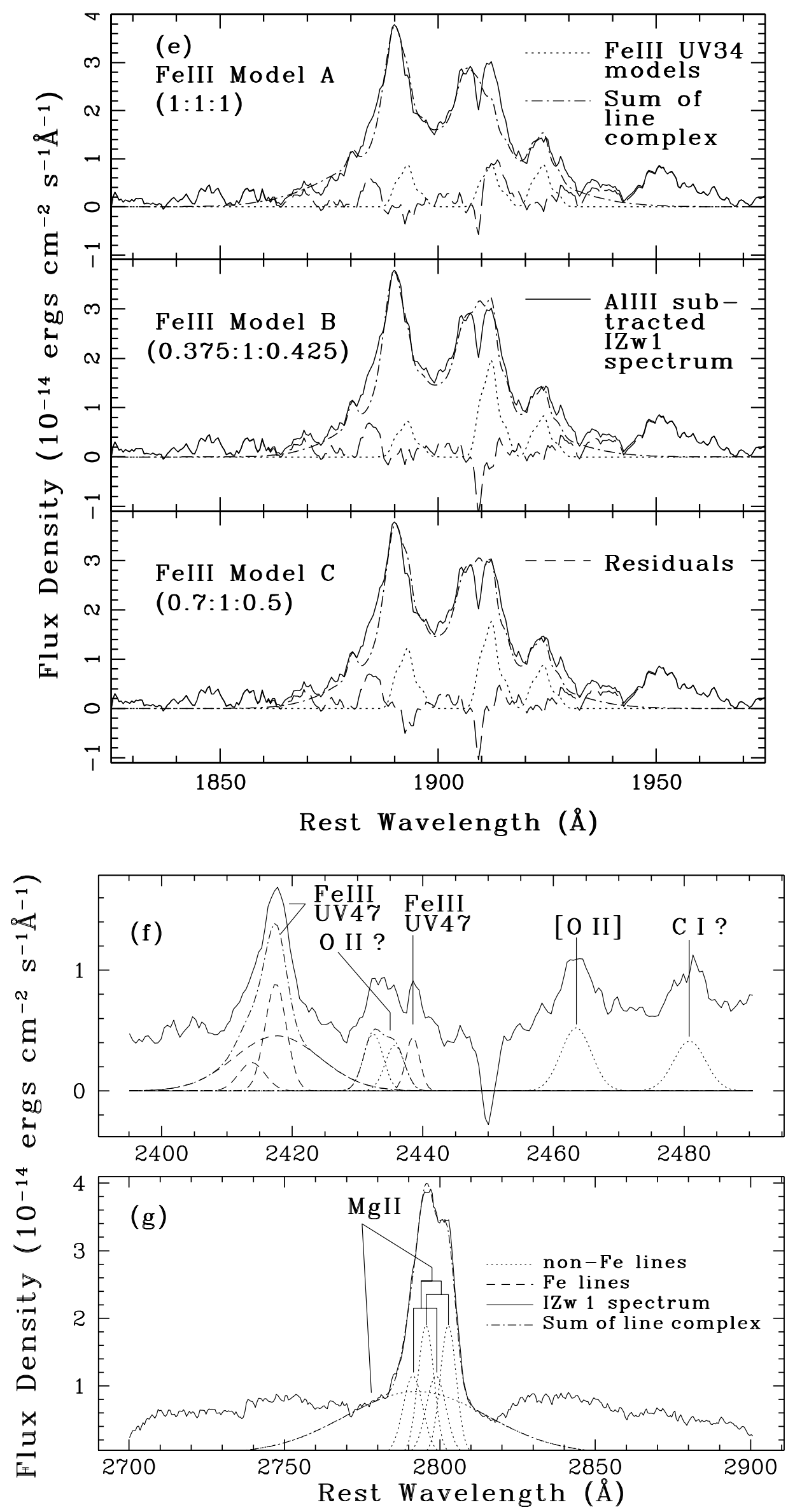

Fig. 5.-Continued 
the non-iron emission is in doubt. We have therefore assumed that the broad component of $\mathrm{Mg}$ II, the existence of which is evident in Figures 1 and $5 g$, dominates this wavelength region, consistent with the findings of L97, and so our template shows a gap in the iron emission around Mg II.

Our sample fitting and subtraction $(\S 4.3)$ of the iron emission in spectra of high-redshift quasars using the template presented here yields residual profiles of the $\mathrm{Mg}$ II line resembling those of the other prominent UV lines, supporting our method. Our Gaussian fitting of $\mathrm{Mg}$ II indicates that one will underestimate its strength by a factor of $\sim 2$ if the broad Mg II component is ignored, as in the interpolation process.

\subsubsection{Synthetic Fe II Spectra}

Wills et al. (1985) present theoretical models of the 1800 $5000 \AA \mathrm{Fe}$ II emission, the Balmer continuum and lines, and compare with observed spectra of a small sample of lowredshift quasars $(z \sim 0.12-0.6)$. Their success in simulating the observed spectra is generally good, though a few discrepancies in line strengths among multiplets remain. Over the past few years the Opacity and IRON projects have applied the power of modern computers to determining the atomic parameters of the thousands of possible iron transitions (e.g., Hummer et al. 1993, Seaton et al. 1994; Nahar et al. 1997, 2000, and references therein), so it is worth revisiting the modeling to see if the deviations of the average Wills et al. Fe II models from the I Zw 1 iron spectrum are now removed. Specifically, Wills et al. predict stronger Fe II emission between $\sim 1800$ and $\sim 2300 \AA$, and weaker Fe II right around $\mathrm{Mg}$ II for $3 \mathrm{C} 273$. In addition, the relative strength of various multiplets appear to differ between their models and the I $\mathrm{Zw} 1$ spectrum. Variations in physical conditions between the NLS1's, of which I Zw 1 is considered the prototype, and the Wills et al. quasars may also explain some of the differences.

Verner et al. (1999) present and discuss their numerical simulations of Fe II emission spectra based on updated iron atomic data, iron line lists (many of which are also used here for line identification), and photoionization modeling. They display the $\mathrm{Fe}$ II emission spectrum for different densities, photon flux, microturbulent velocities below $100 \mathrm{~km} \mathrm{~s}^{-1}$, and iron abundances. Although these line widths are much narrower than those of AGNs and quasars, complicating a comparison, a few differences between the synthetic spectra of Verner et al. and Wills et al. and our empirical Fe II and Fe III templates can immediately be pointed out. (1) The synthetic iron models contain Fe II transitions only, whereas the I Zw 1 spectrum also contain Fe III transitions. (2) The synthetic spectra (Wills et al. 1985; Verner et al. 1999) show a decrease in Fe II emission above $~ 2700-2800$ $\AA$. We are not able to see this in our template as contamination by the Balmer continuum ( $\gtrsim 2800 \AA$ especially) is present. The HST spectrum of I Zw 1 alone does not allow the Balmer continuum contribution to be sufficiently constrained. Complete photoionization modeling is required to properly account for it. However, the Balmer continuum contribution decreases continuously from $\sim 3600 \AA$ down to $\sim 2500 \AA$. Below $\sim 2800 \AA$ it is relatively weak, and so it should not significantly contaminate our $3100 \AA$-limited iron templates.

Detailed comparison between the Fe II template and the most recent Fe II models by Verner et al. (1999) holds great potential for significantly improving our understanding of the iron emitting mechanism and mapping the physical conditions under which the iron emission is radiated. Hence, empirical iron templates remain useful even with the emergence of synthetic iron emission spectra.

\section{APPLICATION OF THE IRON TEMPLATE}

\subsection{Broadening of the Iron Template}

In order to fit and subtract iron emission from any quasar spectrum we need to broaden the iron template to match the line width of that spectrum. This is done by convolving the original template with Gaussian functions of different widths thereby creating a grid of spectra with a range of (quantized) widths.

Three steps are necessary in the iron template broadening process: (1) The standard deviation, $\sigma_{\text {conv }}$, of the convolving Gaussian profile was first estimated using

$$
\begin{aligned}
\sigma_{\text {conv }} & =\mathrm{FWHM}_{\text {conv }} / 2 \sqrt{2 \ln 2} \\
& =\sqrt{\mathrm{FWHM}_{\mathrm{QSO}}^{2}-\mathrm{FWHM}_{\mathrm{IZw} 1}^{2}} / 2 \sqrt{2 \ln 2},
\end{aligned}
$$

where $\mathrm{FWHM}_{\mathrm{IZw} 1}=900 \mathrm{~km} \mathrm{~s}^{-1}$ is the line width of the I Zw 1 spectrum. (2) To apply a constant velocity broadening the computations were carried out in logarithmic wavelength space since $d(\log \lambda)=d \lambda / \lambda=d v / c$. The process of rebinning the broadened template back to linear units results in a small additional broadening. To ensure that the final broadened template has the desired width, we performed broadening simulations on artificial data consisting of a single Gaussian line feature of width $900 \mathrm{~km} \mathrm{~s}^{-1}$, where the width of the convolving Gaussian profile, $\sigma$, was adjusted until the FWHM of the broadened feature, measured in linear wavelength space, matched the desired width to within our measurement errors $\left(\sim 1 \mathrm{~km} \mathrm{~s}^{-1}\right)$. (3) The iron template was then convolved (in logarithmic wavelength space) with a Gaussian profile with the adjusted value of $\sigma$ and rebinned back to linear units.

We emphasize that because quasar spectral lines are often affected by blending, it is important to measure the resulting convolved width using a single artificial spectral line as outlined above, as opposed to estimating the width on the broadened (blended) template itself. We find blending effects to overestimate the line widths by $\sim 15 \%-25 \%$, based on simulated line blends. Isolated narrow line cores, like C IV and $\mathrm{Mg}$ II are, however, expected to be well determined.

\subsection{Cleaning Quasar Spectra for Iron Emission- Application of the Method}

In order to apply this UV iron template to quasar spectra, both the line widths and the iron emission strength of the template must be matched to that of the quasar spectrum. We follow the basic method of estimating the iron emission strength and subtracting the iron emission used by BG92 on the optical (4250-7000 ̊) iron emission of their sample quasars. This method consists of artificially broadening the iron template to a number of widths by convolution with a Gaussian profile $(\S 4.1)$ thereby generating a two-dimensional grid of templates with different line widths. BG92 scaled this "two-dimensional template" by a number of arbitrary, but fixed, strengths, hence creating a three-dimensional grid of templates (with dimensions: wavelength, line width, and line strength). The threedimensional iron template was subtracted from the target spectrum (expanded to a data cube) and the best residual 
(one-dimensional) spectrum was chosen by manual inspection.

Similar to BG92, we created a three-dimensional iron template consisting of five different scalings of a twodimensional template, containing a number of line widths (in the range $1000-15000 \mathrm{~km} \mathrm{~s}^{-1}$ in steps of $250 \mathrm{~km} \mathrm{~s}^{-1}$ is usually sufficient) which are broadened versions of the original iron template (intrinsic width $900 \mathrm{~km} \mathrm{~s}^{-1}$ ). We also performed a manual inspection of the residual ironsubtracted spectra. Our method, however, differs from that of BG92 in the following ways. After fitting and subtracting a power-law continuum fit $^{17}$ to the iron-contaminated quasar spectrum, we iterate to determine the optimum parameters as follows:

1. Determine the iron template (primary) normalization for a given quasar spectrum interactively from its iron emission strength in pure iron emission windows, specified by the user (examples are listed in Table 5). There is a separate normalization for each (one-dimensional) template spectrum with a given broadening.

2. Subtract a (three-dimensional) data cube consisting of five scaled, two-dimensional templates from the target spectrum. The scalings are (five) fractions of the computed normalization and can be chosen arbitrarily. A useful range is $50 \%-150 \%$ of the normalization factor determined above, though the scalings $\neq 100 \%$ are rarely needed except for cross-checks.

3. Compute the residual flux and $\chi^{2}$ of all the fits in the iron emission windows.

4. Manually inspect the residual spectra and pick the best (one-dimensional) iron-subtracted spectrum.

5. Add the previously determined continuum fit for the quasar back into the iron-subtracted spectrum and refit the power-law continuum. (In iron-contaminated spectra only a few narrow, pure continuum windows may exist. After a preliminary subtraction of the iron emission, larger continuum windows can generally be used.)

6. Subtract this (new) continuum fit from the original target spectrum.

7. Determine the new normalizations as in the first item above and repeat all steps iterating over both continuum setting and iron emission strength until both fits (contin-

\footnotetext{
${ }^{17}$ Based on our experience, optimal fitting of the iron emission in any quasar spectrum requires a power-law continuum fit, since a power-law continuum was initially subtracted from the I Zw 1 spectrum before generating the iron template.
}

uum and iron emission) converge and the final ironsubtracted spectrum is satisfactory.

By estimating the template scaling factor interactively, we are not limited to an a priori set of quantized scalings (the quasar spectra can have any normalization and iron emission strength), and we ensure a good starting point for the iron spectrum fitting. Computations of both the $\chi^{2}$ and the residual flux in the user-defined iron emission regions help us to determine the best-fitting iron spectrum objectively. Iterating over both the iron emission strength and the continuum setting improves the fit to them both, especially in heavily contaminated quasar spectra where it may be hard to define pure continuum regions over which the underlying global continuum can be fitted.

\subsection{Sample Iron Emission Subtraction from Quasar Spectra}

We fitted the iron emission in four high-redshift quasars and one low-redshift quasar, demonstrating the successful application of the UV iron template. We briefly summarize our main conclusions and then comment on these results for each individual quasar. The objects and their spectra were chosen to be representative of typically available data for the high-redshift quasars and to contain a range in $\mathrm{Fe}$ II emission strength. The 3C 273 HST spectrum has the advantage of covering the entire UV range. The iron template is also successfully applied to the Large Bright Quasar Survey (Forster et al. 2001). We note that a perfect fit of the $\mathrm{I} Z \mathrm{Ww} 1$ iron emission templates to the iron emission in other AGNs is never expected. This is owing to the strong dependence of the iron emission spectrum on the physical conditions in the emitting gas and on the BLR geometry (Netzer 1980). This is manifested in AGN spectra by variations in the strengths of the different multiplets among individual AGNs (see discussion of 3C 273 below). However, we find the template fitting to work well enough to be a valuable tool for eliminating and studying the iron emission in AGNs at least until accurate theoretical iron emission models are developed.

A reasonable match to the iron emission in the quasar spectra presented here could be obtained by scaling and broadening the combined $\mathrm{Fe}$ II and Fe III UV templates, as described in $\S 4.2$. Similar to the template, Fe II emission is rather common around $\mathrm{Mg}$ II and between $\mathrm{C}$ IV and $\mathrm{C}$ III]. The need to include one or more of the Fe II UV191, Fe III UV34, and Fe III UV47 multiplets, which are strong in I Zw 1 , in the applied template depends on the individual object. Improved iron template fits were generally obtained by sub-

TABLE 5

Pure Iron EMission Windows

\begin{tabular}{|c|c|c|c|c|c|c|c|c|c|c|c|}
\hline \multirow[b]{2}{*}{$\lambda$ LIMITs $^{\mathrm{a}}$} & \multicolumn{11}{|c|}{ RANGe Number } \\
\hline & 1 & 2 & 3 & 4 & 5 & 6 & 7 & 8 & 9 & 10 & 11 \\
\hline$\lambda_{1}(\AA) \ldots \ldots$ & 1350 & 1427 & 1490 & 1705 & $1760^{b}$ & $1930^{c}$ or 1942 & 2250 & $2333^{d}$ & 2470 & 2675 & 2855 \\
\hline$\lambda_{2}(\AA) \ldots \ldots$ & 1365 & 1480 & 1505 & 1730 & 1800 & 2115 & $2320^{\mathrm{d}}$ & $2445^{\mathrm{e}}$ & 2625 & 2755 & 3010 \\
\hline
\end{tabular}

a The precise limits vary from object to object.

b Avoid N III] 21750 line. The width and position may vary from object to object.

c Avoid C III] $\lambda 1909$ broad base if present. If this base is strong, use the 1942-2115 A range.

d Avoid C II] 22326.

${ }^{\text {e }}$ Exclude [Ne IV] $\lambda 2419$ if present. 
dividing the template and scaling each subspectrum (and hence selected groups of multiplets) separately. Good fits were often separately obtained for the Ly $\alpha-\mathrm{C}$ IV, C IV$\mathrm{C}$ III], C III] $-\sim 2300 \AA$, and $\sim 2300-\sim 3100 \AA$ regions.

The most frequent and significant differences in strengths and multiplet ratios of the $\mathrm{Fe}$ II and $\mathrm{Fe}$ III transitions among the objects considered here are in the regions shortward of C IV, between $\sim 1900$ and $\sim 2300 \AA$, and in the Fe II bump around $2500 \AA$. The (undivided) template generally, but not always, overpredicts the 1400-1530 A iron emission in these quasars. ${ }^{18} \mathrm{~A}$ separate (re-) scaling of this iron emission is acceptable because we have no empirical or simple theoretical constraints on the strength of this emission based on the iron emission, say, around Mg II. The variation in the 1900$2300 \AA$ emission strength is possibly due to differing amounts of dust extinction along the line of sight toward individual objects. The general weakness of the 2000-2300

\footnotetext{
18 This may partly be due to the fact that I Zw 1 brightened (§ 2.1) between the $\mathrm{G} 130 \mathrm{H}$ and the $\mathrm{G} 190 \mathrm{H}$ and $\mathrm{G} 270 \mathrm{H}$ spectra were observed and that the iron spectrum does not simply scale with the continuum level.
}

$\AA$ emission in the template suggests that I Zw 1 may be subject to high levels of dust extinction perhaps from its host galaxy and consistent with the observed strong infrared emission (Rieke \& Low 1972; Rieke 1978). The iron multiplet ratios may also (or instead) vary significantly in this region among quasars. This discrepancy is not unique to the quasars presented here (Figs. 6 and 7) but is also seen in other broad-lined quasars (e.g., Wills et al. 1985; Steidel \& Sargent 1991; Corbin \& Boroson 1996; M. Vestergaard et al. 2001, in preparation). Residual emission is similarly present in the sample iron-subtracted spectrum of Corbin \& Boroson (1996; their Fig. 2).

The overall and important conclusion is that a subdivision of the $\sim 2000 \AA$ wide Fe II and Fe III UV templates may often be necessary because the individual multiplet strengths across the spectrum vary between objects. Similarly, the Fe II and Fe III templates may require different scalings as evidenced by the absence of $\mathrm{Fe}$ III emission in some sources (e.g., Q1451 + 1017; Baldwin et al. 1996). Also, there is no strong direct coupling expected between the UV $\mathrm{Fe}$ III and $\mathrm{Fe}$ II emission. The $\mathrm{Fe}$ II is emitted from the

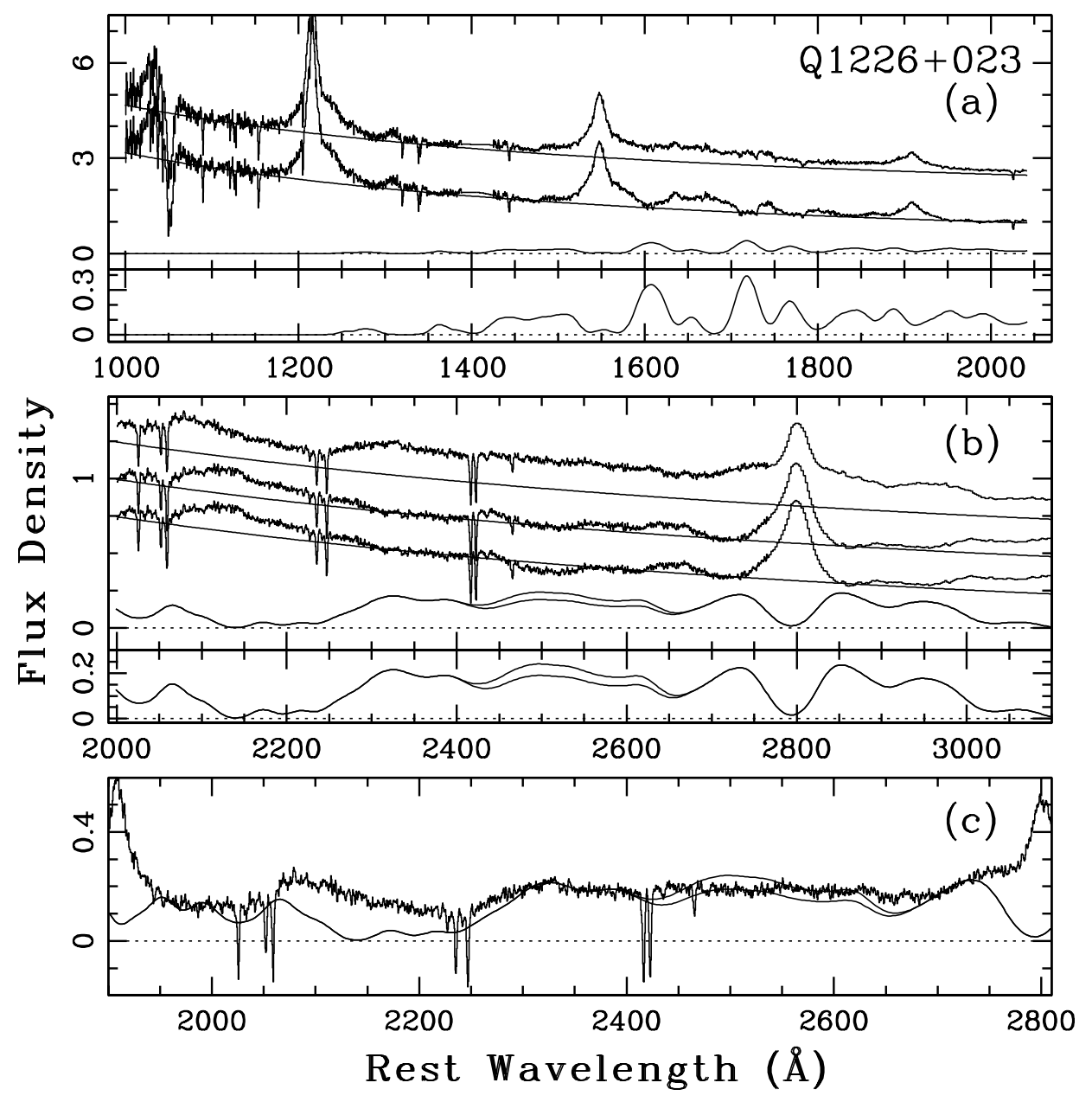

FIG. 6. - HST spectrum of 3C 273 (Q1226+023) and the best-fit iron "models." The upper, large panel in (a) shows the original spectrum (shifted for clarity; see $\S 4.3$ ) with the continuum overplotted (top) compared to the residuals (middle) after subtracting the best-fit iron "model” (bottom). The latter is displayed separately below the large panel for clarity. Panel $(b)$ shows the original spectrum, two iron "model" fits, and the corresponding spectrum residuals. Neither of the iron "models" fits the $\sim 2500 \AA$ multiplets very well, but they fit reasonably well to the blue and red part of the "multiplet", respectively. Panel (c) shows these iron "models" more clearly. See $\S 4.3$ for more details. The flux density is in units of $10^{-13} \mathrm{ergs} \mathrm{cm}^{-2} \mathrm{~s}^{-1} \AA^{-1}$. 

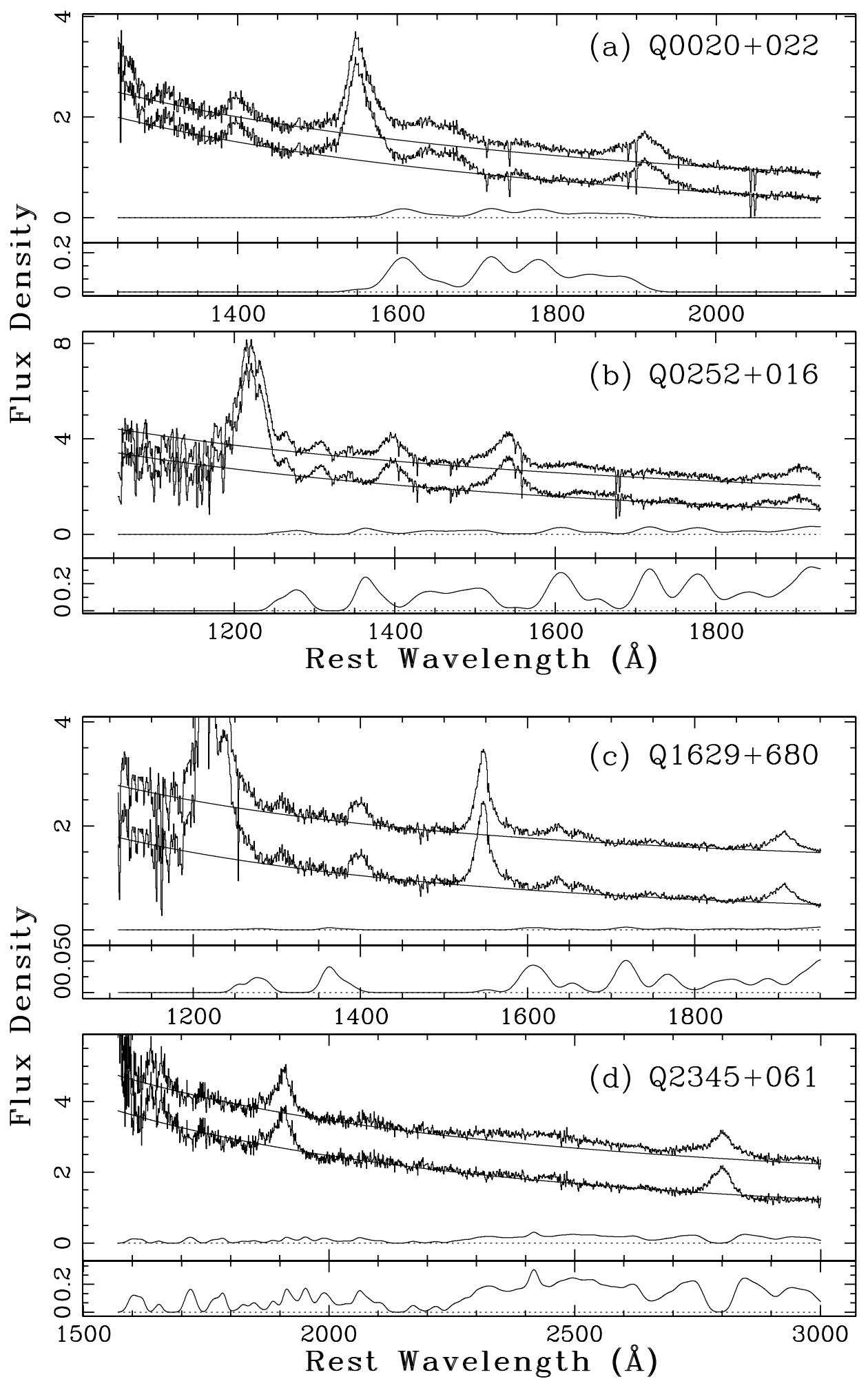

FIG. 7.- Sample fitting of the iron emission in typical high-redshift quasar spectra. In the upper, large panels the original spectrum (top) with the power-law continuum fit, is compared to the residual spectrum (continuum superposed; middle) after subtracting the best-fit iron emission model (bottom). In the lower, smaller panels the iron models are plotted separately for visibility. Q0020+022 and Q0252+016 display pronounced "pseudo-continuum" in the 1500-1900 Å range. The data are from M. Vestergaard et al. (2001, in preparation) with flux density in units of $10^{-16} \mathrm{ergs} \mathrm{cm}^{-2} \mathrm{~s}^{-1} \AA^{-1}$. See $\S 4.3$ for continuum parameters and details on the relative spectrum shifts valid for each quasar.

partially ionized hydrogen zone while the Fe III originates in the fully ionized zone. Some correlation is expected to exist as both ions are (at least partly) excited by the UV continuum, but the current theoretical models are not accurate enough to firmly establish a simple scaling relation between the strong multiplets of UV Fe II and Fe III, if one exists (A. Pradhan 2000, private communication). Therefore, a separate scaling of the UV Fe II and Fe III emission tem- 
plates is allowed in order to optimize the fit to the AGN iron emission. However, this was not necessary for the AGNs presented here.

We first present the fitting results on $3 \mathrm{C} 273$ as its 940 $3200 \AA H S T$ spectrum allows the full range of the template to be tested on a single AGN.

$$
\text { 4.3.1. } Q 1226+023 ; 3 C 273(z=0.157)
$$

The 3C 273 spectrum (Fig. 6) does not contain any obvious features of the Fe II UV191, Fe III UV34, and Fe III UV47 multiplets so the spectrum was fit with templates excluding these features. The combined $\mathrm{Fe}$ II and $\mathrm{Fe}$ III template did a good job, once subdivided into five independently scaled segments (at 1540, 1912, 2423, and $2656 \AA$ ). A scaling of the full range template to the iron emission redward of $\mathrm{C}$ III] and in the small blue bump yielded consistent flux levels blueward of $\mathrm{C}$ IV if no $\mathrm{N}$ IV] $\lambda 1486$ line is present. We could not distinguish between this fit and one with a separate scaling and a $\mathrm{N}$ IV] line (the latter fit is shown in Fig. 6). The fitted iron model in the range between $\mathrm{C}$ IV and $\mathrm{C}$ III] yields residuals coinciding with the expected wavelengths of known lines, such as $\mathrm{He}$ II $\lambda 1640, \mathrm{O}$ III] $\lambda 1664, \mathrm{Al}$ II $\lambda 1670, \mathrm{~N}$ III] $\lambda 1750, \mathrm{Si}$ II $\lambda 1814$, and $\mathrm{Al}$ III $\lambda 1857$ yielding confidence in the template and the model fit. We found the $\mathrm{Mg}$ II profile in the iron subtracted 3C 273 spectrum to show a slightly blue asymmetric profile. This profile could be fitted very well with two Gaussian functions with the same FWHM of $3800 \mathrm{~km} \mathrm{~s}^{-1}$ (one shifted $-3400 \mathrm{~km}$ $\mathrm{s}^{-1}$ from the narrow peak of $\mathrm{Mg}$ II; FWHM is consistent with the widths of the UV lines and the narrow Balmer lines, $\sim 4000 \mathrm{~km} \mathrm{~s}^{-1}$ ). This gives us confidence that the iron template in the $\mathrm{Mg}$ II region is representative of the average Fe II bump in AGNs.

The 3C 273 spectrum shows stronger iron emission than I $\mathrm{Zw} 1$ between the $\mathrm{C}$ IV and $\mathrm{C}$ III] lines and in the 2000$2300 \AA$ range (Fig. 6) relative to the remaining UV iron emission. As the I $\mathrm{Zw} 1$ template does not contain much iron emission in the 2100-2250 ̊ range, no further attempt to model this additional emission is possible with the current template. The I Zw 1 template clearly overestimates the $3 \mathrm{C} 273$ iron strength around $2500 \AA$. The emission is rather flat across this region contrary to that in the template. Two different attempts to model this region were made (Figs. $6 b$ and $6 c$ ). One "model" fits the blue part while the other fits the red part, but neither does a good overall job. The original template has much stronger emission at $2500 \AA$ than that shown in Figure 6. In the case of the $\sim 2100$ and $\sim 2500 \AA$ "bumps" a different template is clearly needed to account for this emission. Redward of $\mathrm{Mg}$ II the template shows a similar shape as the observed $\mathrm{Fe}$ II but slightly underestimates the emission. It is likely due to a slight overestimation of the underlying continuum level in I $\mathrm{Zw} 1$ owing to the truncation of its spectrum at $3089 \AA$ rest frame by the FOS G270H grating.

The power-law ${ }^{19}$ continuum has $F_{\lambda}(1500 \AA)=1.608$ $\times 10^{-13}$ ergs $\mathrm{cm}^{-2} \mathrm{~s}^{-1} \AA^{-1}$ and slope, $\alpha_{\lambda}=1.67\left(\alpha_{v}=\right.$ 0.33 ). The original spectrum is shifted by $+1.5 \times 10^{-13} \mathrm{ergs}$ $\mathrm{cm}^{-2} \mathrm{~s}^{-1} \AA^{-1}$ in Figure $6 a$ and by $+0.25 \times 10^{-13}$ ergs $\mathrm{cm}^{-2} \mathrm{~s}^{-1} \AA^{-1}$ in Figure $6 b$. In this figure the residuals from the "blue $2500 \AA$ ” iron model, shown in the middle, represents the original flux level of the spectrum. The residuals from the "red $2500 \AA$ " iron model, shown as the lowest of

\footnotetext{
${ }^{19} F_{v} \sim v^{-\alpha_{v}}$ and $F_{\lambda} \sim \lambda^{-\alpha_{\lambda}} ; \alpha_{\lambda}=2-\alpha_{v}$ (e.g., Weedman 1986).
}

the three spectra in panel (b), is shifted by $-0.25 \times 10^{-13}$ ergs cm $\mathrm{cm}^{-2} \mathrm{~s}^{-1} \AA^{-1}$. Figure $6 c$ shows the two $2500 \AA$ iron models more clearly; the spectrum and the models are continuum subtracted here. The iron model has a FWHM of $4000 \mathrm{~km} \mathrm{~s}^{-1}$. The processing of the HST data is outlined in Appendix B.

\subsection{2. $Q 0020+022(z=1.798)$}

There is no clear indication of strong iron emission in the $1250-1500 \AA$ range, and the continuum level is relatively lower than the flux level in the 1600-1900 $\AA$ range. The flatness of the 1930-2050 ̊ region also indicates very weak iron emission, so this region was adopted as a continuum region. The power-law continuum has normalization, $F_{\lambda}(1500 \AA)=1.753 \times 10^{-16} \mathrm{ergs} \mathrm{cm}^{-2} \mathrm{~s}^{-1} \AA^{-1}$ and slope, $\alpha_{\lambda}=1.94\left(\alpha_{v}=0.06\right)$. The combined Fe II and Fe III template was fitted to the data between 1530 and $1900 \AA$ only (Fig. 7a) yielding residuals which coincide very well with the expected positions of $\mathrm{He}$ II $\lambda 1640, \mathrm{O}$ III] $\lambda 1664, \mathrm{Al}$ II $\lambda 1670$, $\mathrm{N}$ III] $\lambda 1750$, and Si II $\lambda 1814 \AA$. The residual spectrum is shifted by $-0.5 \times 10^{-16}$ ergs $\mathrm{cm}^{-2} \mathrm{~s}^{-1} \AA^{-1}$. The iron model has FWHM of $6500 \mathrm{~km} \mathrm{~s}^{-1}$.

$$
\text { 4.3.3. } Q 0252+016(z=2.457)
$$

An improved fit to the iron emission (Fig. $7 b$ ) could be obtained by including the Fe II UV191 $\lambda 1786$ and Fe III UV34 $\lambda \lambda 1895,1914,1926$ features in the templates and by separately fitting the $1400-1550 \AA$ emission which is weaker than in the UV template, but still significant. It is unclear whether weak Fe II emission still remains in the blue wing of C IV $\lambda 1549$, though both wings of this line indicate the presence of a somewhat broad underlying component. The power-law continuum has $F_{\lambda}(1500 \AA)=2.807 \times 10^{-16} \mathrm{ergs}$ $\mathrm{cm}^{-2} \mathrm{~s}^{-1} \AA^{-1}$ and slope, $\alpha_{\lambda}=1.28\left(\alpha_{v}=0.72\right)$. The residual spectrum is shifted by $-1.0 \times 10^{-16}$ ergs cm $\mathrm{cm}^{-2} \mathrm{~s}^{-1} \AA^{-1}$. The iron model has FWHM of $5000 \mathrm{~km} \mathrm{~s}^{-1}$.

$$
\text { 4.3.4. } Q 1629+680(z=2.478)
$$

The iron emission is relatively weak (Fig. $7 c$ ) compared to the other quasars presented here, as indicated by the lower emission level between C IV and C III]. Based on the C IV line width of FWHM $\sim 4000 \mathrm{~km} \mathrm{~s}^{-1}$, a reasonable but weak model fit could be made using the combined $\mathrm{Fe}$ II and $\mathrm{Fe}$ III templates in which Fe II UV191 $\lambda 1786$ and Fe III UV34 $\lambda \lambda 1895,1914$ were excluded. One exception is the region between $\lambda 1400$ and C IV, where the UV template strongly overpredicts the $\mathrm{Fe}$ II emission. In fact, the data do not indicate noticeable $\mathrm{Fe}$ II emission there. Thus, the iron emission was fitted using a version of the template in which the $1400-1550 \AA$ iron emission is excluded. The power-law continuum has $F_{\lambda}(1500 \AA)=8.903 \times 10^{-17} \mathrm{ergs} \mathrm{cm}^{-2} \mathrm{~s}^{-1}$ $\AA^{-1}$ and slope, $\alpha_{\lambda}=2.3\left(\alpha_{v}=-0.3\right)$. The original spectrum is shifted by $+1.0 \times 10^{-16}$ ergs $\mathrm{cm}^{-2} \mathrm{~s}^{-1} \AA^{-1}$. The iron model has FWHM of $4000 \mathrm{~km} \mathrm{~s}^{-1}$.

$$
\text { 4.3.5. } Q 2345+061(z=1.540)
$$

A scaling and broadening of the combined $\mathrm{Fe}$ II and $\mathrm{Fe}$ III templates (with Fe II UV191 $\lambda 1786$ excluded) to $\sim 2500 \mathrm{~km}$ $\mathrm{s}^{-1}$ offer a reasonable overall fit to the iron line emission (Fig. 7d). Hence, no subdivision of the UV template was performed. The general shape of the iron emission longward of $\mathrm{Mg}$ II is reproduced by the template but a slight drop in the emission level is seen in the quasar data owing to the enhanced calibration uncertainty in this region where second order light merges with the primary order spectrum. 
The main bumps and wiggles in the Fe II bump around $\mathrm{Mg}$ II can be reproduced with the exception of the $\sim 2300$ $2500 \AA$ region where excess emission is clearly present. Including the Fe III UV47 $\lambda \lambda 2418,2438$ feature in the template (shown in Fig. 7d) does not fully account for the iron emission in this region. Excess emission is also clearly present at $\sim 2050-2150 \AA$. A good coincidence of the iron strength is found for $\sim 1800-2000 \AA$, based on a scaling of the template $\mathrm{Fe}$ II bump around $\mathrm{Mg}$ II to the data, in spite of the larger noise level below $2100 \AA$. The large noise levels at $\lambda<1800 \AA$ renders the discrepancies of the data with the template fit irrelevant. The power-law continuum has $F_{\lambda}(1600 \AA)=3.62 \times 10^{-16}$ ergs cm ${ }^{-2} \mathrm{~s}^{-1} \AA^{-1}$ and slope, $\alpha_{\lambda}=1.72\left(\alpha_{v}=0.28\right)$. The original spectrum is shifted by $+1.0 \times 10^{-16}$ ergs cm $\mathrm{cm}^{-2} \mathrm{~s}^{-1} \AA^{-1}$. The iron model has FWHM of $2500 \mathrm{~km} \mathrm{~s}^{-1}$.

\section{DISCUSSION OF THE I ZW 1 SPECTRUM}

The spectrum of $\mathrm{I} Z \mathrm{~W} 1$ is rich in emission lines, and in particular this high S/N HST spectrum reveals a plethora of weaker lines (L97; Fig. 1) not usually seen in quasar spectra. The spectrum has strong low-ionization lines while some of the higher ionization lines are weaker than expected for an average quasar (Francis et al. 1991; Zheng et al. 1997; Wilkes 2000; L97 and references therein; see discussion on $\mathrm{Si}$ IV + O IV] and C Iv below). Both [O III] $\lambda 5007$ (BG92) and C III] $\lambda 1909$ (this spectrum) are weak.

While a detailed emission line study is not the subject of this paper, we briefly discuss our results in relation to earlier work on this object.

\subsection{Line Parameters}

5.1.1. Line Widths

Our measurements and line fitting show that the widths of the fitted iron emission components are rather narrow; ranging from $\sim 300$ to $900 \mathrm{~km} \mathrm{~s}^{-1}$. For example, the narrow line core of Fe II UV191 $\lambda 1786$ has FWHM of 550 $\mathrm{km} \mathrm{s}^{-1}$ and a similar fit to Fe III UV47 $\lambda 2418$ yields a FWHM of $715 \mathrm{~km} \mathrm{~s}^{-1}$ (Table 4). This narrow width may be an intrinsic property of the iron emission mechanism or an effect of the continuum level uncertainty (the line width will appear narrower if the continuum is set too high). Our narrowest line measurements of $\sim 300-400 \mathrm{~km} \mathrm{~s}^{-1}$ of the weaker non-iron lines are consistent with the findings of van Groningen (1993), who concluded, based on work by himself and Phillips (1976), that I Zw 1 has three emitting regions, one of which has a velocity dispersion of $\sim 400 \mathrm{~km}$ $\mathrm{s}^{-1}$.

\subsubsection{Line Velocity Shifts}

The peaks of the broad emission lines are blueshifted (Table 3) relative to the systemic redshift $\left(z_{\mathrm{em}}=0.061\right)$, defined by the $\mathrm{Mg}$ II doublet (Appendix $\mathrm{A}$ ) and $\mathrm{H}$ I radio measurements (Condon et al. 1985). The high-ionization lines have a higher blueshift (average shift $\sim 1540 \pm 500 \mathrm{~km}$ $\left.\mathrm{s}^{-1}\right)$ than the low-ionization lines $\left(\sim 500 \pm 270 \mathrm{~km} \mathrm{~s}^{-1}\right.$; see also Table 3, L97, and below for exceptions).

A significant amount of blueshifted emission is evident in lines such as $\mathrm{N}$ v, the $\mathrm{C}$ IV $+\mathrm{Si}$ II blend, and $\mathrm{He}$ II $\lambda 1640$ (Figs. $5 a, 5 c$, and 1, respectively). A special case is that of He II where practically all the emission is blueshifted, reaching $\sim 1300-2200 \mathrm{~km} \mathrm{~s}^{-1}$ relative to the quasar rest frame (see also L97). The $\mathrm{N} v$ line profile can be fitted as two emission components originating at different velocity shifts, $\sim-900 \mathrm{~km} \mathrm{~s}^{-1}$ and $\sim-2400 \mathrm{~km} \mathrm{~s}^{-1}$ (Fig. 5a, Table 3).
The $\sim 900 \mathrm{~km} \mathrm{~s}^{-1}$ blueshift of $\mathrm{N} \mathrm{v}$ is similar to that of the narrow $\mathrm{C}$ IV component, the broad $\mathrm{Al}$ III component, and one of the narrow components of $\mathrm{Ly} \alpha$ and $\mathrm{Si}$ IV $+\mathrm{O}$ IV], respectively. Most of our line shifts are consistent with those of L97 to within the errors and the spectral resolution (Leitherer 1995; Tables 3 and 4). We suspect the velocity shift differences $\lesssim 1300 \mathrm{~km} \mathrm{~s}^{-1}$ with L97 for Si II $\lambda 1263$, Si II $+\mathrm{O}$ I $\lambda 1306, \mathrm{Si}$ IV $+\mathrm{O}$ IV] $\lambda 1400$, and N III] $\lambda 1750$ can be explained by a combination of uncertainties in the line positions of $\lesssim 0.5-1 \AA$ (i.e., $250-500 \mathrm{~km} \mathrm{~s}^{-1}$; conservative error), resulting from the heavy line blending present in both studies, and the slightly degraded resolution in our $\mathrm{G} 130 \mathrm{H}+\mathrm{G} 190 \mathrm{H}$ spectra (§ 2).

L97 suggested an outflowing component is responsible both for the blueshifted line peaks (of both low and high ionization), the blueshifted ( $2000 \mathrm{~km} \mathrm{~s}^{-1}$ ) emission (blue wing asymmetry) and the associated weak UV absorption in $\mathrm{Ly} \alpha, \mathrm{N} v$, and $\mathrm{C}$ IV which they detect.

\subsubsection{Line Strengths}

L97 use the doublet ratios of $\mathrm{Mg}$ II and $\mathrm{Al}$ III to deduce the location of their emitting regions relative to the "outer BLR boundary." As discrepancies are seen between our line measurements and those of L97, it is of interest to briefly discuss the reasons and the implications for their BLR size results. At $\lesssim 1500 \AA$ the main differences are due to the fact that L97 does not scale the G130H spectrum to match the level of the $\mathrm{G} 190 \mathrm{H}$ and $\mathrm{G} 270 \mathrm{H}$ spectra, as done here.

The line measurements at $\geq 1500 \AA$ are different in part due to different Gaussian fitting techniques (cf. Laor et al. 1994), but mostly due to L97's use of a local continuum (A. Laor 1997, private communication) as opposed to a global one, as done here except at the Fe II bump, 2300-3090 А. The EW measurements in regions of isolated and unblended emission lines (where the continuum is well determined) agree well (C II $\lambda 1335$, the $\lambda 1345$ feature, and $\mathrm{Si}$ II 21814). However, for the remaining lines (see Table 1 in L97 and Table 3, this work), the EW measurements differ by $-75 \%$ to $180 \%$ and the line fluxes by $-70 \%$ to $215 \%$, where the faint or blended lines deviate the most $(|\Delta \mathrm{EW}|>30 \%$ and $\mid \Delta$ (line flux) $\mid>30 \%$ in lines such as Si II $\lambda 1260$ [blended], O III] $\lambda 1664, \mathrm{Al}$ II $\lambda 1670, \mathrm{Fe}$ II $\lambda 1786$, Al III $\lambda \lambda 1854,1863, \mathrm{~N}$ II] $\lambda 2141, \mathrm{C}$ II] 22326 ). These discrepancies measured from the same data illustrate the significant uncertainties associated with line and continuum fitting in quasar spectra.

We can not easily confirm the suggestion by L97 that Al III $\lambda \lambda 1854,1863$ can probe the BLR size, as it appeared only marginally thermalized (ratio $=1.25: 1$ ). Our measurements of the narrow $\mathrm{Al}$ III doublet ${ }^{20}$ (ratio $\left.=0.9: 1\right)$ suggests it is thermalized, possibly being emitted somewhat closer to the continuum source, i.e., inside the BLR outer radius. We also find $\mathrm{Mg}$ II to be (entirely) thermalized (doublet ratio $=1: 1) ; \mathrm{L} 97$ measure a ratio $=1.2: 1$. Contrary to L97 we find a much better overall fit to the narrow $\mathrm{Mg}$ II emission using more than two components; four components are required to ensure an equal line width in each doublet (Table 3). Thus, it appears that neither $\mathrm{Mg}$ II nor Al III is a suitable probe of the outer boundary of the BLR (see discussion by L97).

\footnotetext{
${ }^{20} \mathrm{The} \mathrm{Al}$ III doublet components have equal widths to within the spectral resolution.
} 


\subsection{The $\mathrm{C}$ III] Complex}

The density-sensitive lines, C III] $\lambda 1909$ and Si III] $\lambda 1892$ are important BLR density diagnostics. At first sight their relative strengths in I Zw 1 are highly unusual: $\mathrm{C} \mathrm{III]}$ is $\sim 10$ times weaker and $\mathrm{Si}$ III] significantly stronger ${ }^{21}$ than usual leading L97 to argue for unusual high densities $\left(\sim 10^{11}\right.$ $\left.\mathrm{cm}^{-3}\right)$. However, in reality this comparison is difficult to make. Published measurements of C III] line strengths (e.g., Wilkes 1986; Francis et al. 1991; Baldwin et al. 1995) are generally measurements of the entire $\mathrm{C} \mathrm{III]} \mathrm{complex} \mathrm{includ-}$ ing $\mathrm{Si}$ III], $\mathrm{Al}$ III, and Fe III emission, which are all strong in I Zw 1. In typical quasars with much larger intrinsic line widths these lines are so heavily blended that detailed deblending techniques often cannot uniquely determine the relative contributions from individual transitions. Note, if Si III] and Fe III are both strong at high densities the complex would not necessarily appear asymmetric at FWHM $\gtrsim 3500 \mathrm{~km} \mathrm{~s}^{-1}$. By artificially broadening the I ZW 1 spectrum to FWHM of 3000 and $5000 \mathrm{~km} \mathrm{~s}^{-1}$, a typical range for quasars, we estimate the $\mathrm{C}$ III] line strength measurements (line flux and $\mathrm{EW}$ ) in a similar, broad-lined source would be overestimated ${ }^{22}$ by a factor of $\sim 2$.

Detailed modeling of the full UV spectra of other NLS1's also concludes that these sources have high-density emitting regions $\left(\sim 10^{11}-10^{12} \mathrm{~cm}^{-3}\right.$, Kuraszkiewicz et al. 2000), lending support to L97's conclusions. Given that the strong Fe III UV34 multiplet is severely blended with the $\mathrm{C}$ III] and Si III] emission lines, we note that it is likely that both L97 and Kuraszkiewicz et al. overestimate the strength of the Si III] line as they underestimate the strength of the Fe III UV34 $1895 \AA$ transition. The combination of a resulting smaller $\mathrm{Si}$ III]/C III] ratio and strong Fe III and Al III contributions will in the framework of photoionization models (e.g., Fig. 3e, Korista et al. 1997) then further strengthen their conclusions that the BLR densities, at least in NLS1's, are very high.

A theoretical estimate of the relative triplet strength for the Fe III UV34 $\lambda \lambda 1895,1914,1926$ transitions for AGN physical conditions is not readily available. The relative significances of the possible excitation mechanisms are not fully established in part due to the complexity of the Fe II and Fe III emission. The Fe III UV34 excitation mechanism is probably not due to electron impacts owing to the high energy levels of the multiplet transitions (several eV) compared to the (typical) plasma temperature of $\sim 10^{4} \mathrm{~K}(\sim 1$ $\mathrm{eV})$. The multiplet could be due to fluorescence and/or photoionization/recombination, as is likely to be the case for the UV Fe III emission in general (A. Pradhan 2000, private communication). In the optically thick regime all the transitions should have equal strengths due to thermalization (e.g., L97). Hartig \& Baldwin (1986, hereafter HB86) estimate the relative Fe III UV34 multiplet strengths by fitting the $\lambda \lambda 1895,1914,1926$ transitions in the spectrum of H0335-336 where this multiplet dominates the Si III] and $\mathrm{C} \mathrm{III]} \mathrm{emission.} \mathrm{Assuming} \mathrm{H} 0335-336$ has no Si III] and $\mathrm{C}$ III] emission at all, they find a multiplet ratio of $0.9: 1: 0.3$, while including $\mathrm{Si}$ III] and $\mathrm{C} \mathrm{III]}$ in their fits yields a relative flux ratio of $0.9: 1: 0.7$. The $\mathrm{I} Z \mathrm{Zw} 1$ spectrum is consistent with either of these ratios, as we discuss next.

\footnotetext{
${ }^{21}$ The critical density of $\left.\mathrm{Si} \mathrm{III}\right]$ is $1.1 \times 10^{11} \mathrm{~cm}^{-3}$ (Baldwin et al. 1996; L97).

${ }^{22}$ The $\mathrm{Al}$ III line is excluded in the comparison.
}

In order to estimate the likely contribution to the $\mathrm{C}$ III] complex from the Fe III UV34 triplet, we modeled this multiplet with special emphasis on the relative transition strength, on varying its contribution and noting the effect on the $\mathrm{Si}$ III] and $\mathrm{C} \mathrm{III]} \mathrm{model} \mathrm{fits} \mathrm{(see} \mathrm{Table} \mathrm{6).} \mathrm{We} \mathrm{adopted}$ the approach by $\mathrm{HB} 86$, who use the emission profile of $\mathrm{Fe}$ II UV191 to model the Fe III triplet (J. A. Baldwin 2000, private communication). We note that due to the strong blending in the $\mathrm{C}$ III] line complex and the nonorthogonality of Gaussian functions, the model fits are not unique. This is also clear from the fact that a range of reasonable model fits to the Fe III UV34 emission can be made. Assuming there is no $\mathrm{Si}$ III] and $\mathrm{C} \mathrm{III]}$ emission at all (or broad emission thereof at least) a triplet ratio of 0.91:1:0.46 can be fitted (not shown). However, the significant residuals at 1890 and $1907 \AA$ A strongly argue that Si III] and $\mathrm{C} \mathrm{III]} \mathrm{emission} \mathrm{is} \mathrm{present.} \mathrm{Several} \mathrm{fits} \mathrm{were} \mathrm{made} \mathrm{both}$ where the triplet ratio was fixed at 1:1:1 and where it was allowed to vary freely. Sample fits are shown in Figure $5 e$ and Table 6 (Note that the triplet is located at $\lambda \lambda 1893,1912$, 1924). The best fits were found for triplet models where the $1914 \AA$ transition is the strongest. We cannot distinguish between the various fits nor between the optically thick and thin cases. More advanced modeling is required. A reasonable approach in fitting the Fe III in this region until more is known about the UV34 triplet may be as follows. If $\mathrm{Al}$ III is strong, Fe III (including UV34) is highly likely to be present and similarly strong (HB86). If so, two fits can be made: one ignoring the presence of Fe III UV34, and one in which its contribution is maximized. This allows an upper limit to the fitting uncertainty involved to be estimated.

In Figures 2, 3, and $5 d$ the Fe III template is shown with the UV34 triplet ratio 0.375:1:0.425 (model B) for illustrative purposes only. We note that other strong Fe III transitions may be present in the $\mathrm{C}$ III] complex in addition to Fe III UV34, as indicated in Figure 4.

\subsection{The $\mathrm{Si} \mathrm{IV}+\mathrm{O} \mathrm{IV}] \lambda 1400$ and $\mathrm{C}$ IV Features}

The Si IV $+\mathrm{O}$ IV] $\lambda 1400$ feature is strong relative to $\mathrm{C}$ IV $(\lambda 1400 / \mathrm{C}$ IV $\sim 1.3$; Table 3$)$, and the $\mathrm{C}$ IV/Ly $\alpha$ ratio $(0.11)$ is also low compared to the typical values for quasars: $0.1-0.6$ and 0.2-0.6, respectively (Wilkes 1986; Francis et al. 1991; Baldwin et al. 1995). We note that $\lambda 1400 / C$ IV measured from the nonscaled $\mathrm{G} 130 \mathrm{H}$ spectrum $(\S 2.1)$ is also unusually strong $(\sim 1)$. The line lists by Nahar $(1995)$ and Nahar \& Pradhan (1996) indicate a (conservative) upper limit to the iron emission contribution to the $\lambda 1400$ feature of $\sim 20 \%$. Thus, the $\lambda 1400 / \mathrm{C}$ IV line ratio indicates a density $n_{e} \gtrsim 10^{11} \mathrm{~cm}^{-3}$ according to the models by Rees, Netzer, \& Ferland (1989), consistent with the estimate by L97 of $n_{e} \sim$ $10^{11} \mathrm{~cm}^{-3}$ and typical NLS1 densities (Kuraszkiewicz et al. 2000). At high densities a simultaneous strengthening of the Silicon lines and weakening of the Carbon lines occurs (e.g., Rees et al. 1989).

Another contributor to the large $\lambda 1400 / C$ IV intensity ratio may be an ionization effect due to the unusually red spectral energy distribution of I Zw 1 [e.g., similar to the explanation by Zheng \& Malkan (1993) of the Baldwin effect (Baldwin 1977)]. The luminosity brightening relative to the IUE data measurements $(\S 2)$ appears to have increased these line ratios in agreement with Zheng \& Malkan (1993), with the C IV complex flux increasing the least $(\sim 75 \%$; Ly $\alpha$ increased by $\sim 110 \%$; Wu et al. 1983 ; Wang et al. 1996; Rodríguez-Pascual et al. 1997). 
TABLE 6

Model Fit Measurements of Fe III UV34, [Si III], Si III], AND C III]

\begin{tabular}{|c|c|c|c|c|c|}
\hline Line & $\begin{array}{l}\lambda_{\text {lab }} \\
(\AA)\end{array}$ & $\begin{array}{c}\text { Line Flux } / 10^{-14} \\
\left(\text { ergs } \mathrm{cm}^{-2} \mathrm{~s}^{-1} \AA^{-1}\right)\end{array}$ & $\begin{array}{l}\text { EW } \\
(\AA)\end{array}$ & $\begin{array}{l}\text { FWHM } \\
\left(\mathrm{km} \mathrm{s}^{1}\right)\end{array}$ & $\begin{array}{l}\text { Flux } \\
\text { Ratio }\end{array}$ \\
\hline \multicolumn{6}{|c|}{ Model A } \\
\hline \multirow[t]{3}{*}{ Fe III UV34 ......... } & 1895.5 & 4.55 & 1.5 & $\ldots$ & 1.0 \\
\hline & 1914.1 & 4.50 & 1.5 & $\ldots$ & 1.0 \\
\hline & 1926.3 & 4.50 & 1.5 & $\ldots$ & 1.0 \\
\hline Fe III Sum ........... & $\ldots$ & 13.55 & 4.5 & $\ldots$ & $\ldots$ \\
\hline$[\mathrm{Si}$ III $] \ldots \ldots \ldots \ldots \ldots$ & 1882.7 & 0.92 & 0.3 & 350 & \\
\hline \multirow{2}{*}{ Si $\mathrm{III}] \ldots \ldots \ldots \ldots \ldots \ldots$} & 1892.0 & 11.46 & 3.7 & 830 & \\
\hline & 1892.0 & 19.86 & 6.5 & 3960 & \\
\hline$[\mathrm{Si} \mathrm{III}]+\mathrm{Si} \mathrm{III}] \ldots .$. & $\ldots$ & 32.24 & 10.5 & $\ldots$ & \\
\hline \multirow[t]{2}{*}{$\mathrm{C}$ III $] \ldots \ldots \ldots \ldots \ldots$} & 1908.7 & 9.5 & 3.1 & 875 & \\
\hline & 1908.7 & 40.77 & 13.4 & 5500 & \\
\hline \multirow[t]{2}{*}{ C III] Sum ........... } & $\ldots$ & 50.26 & 16.5 & $\ldots$ & \\
\hline & & Model $\mathrm{B}^{\mathrm{a}}$ & & & \\
\hline \multirow[t]{3}{*}{ Fe III UV34 ......... } & 1895.5 & 3.76 & 1.2 & $\ldots$ & 0.375 \\
\hline & 1914.1 & 10.04 & 3.3 & $\ldots$ & 1.0 \\
\hline & 1926.3 & 4.24 & 1.4 & $\ldots$ & 0.425 \\
\hline Fe III Sum ........... & $\ldots$ & 18.04 & 5.9 & $\ldots$ & $\ldots$ \\
\hline$[\mathrm{Si}$ III $] \ldots \ldots \ldots \ldots \ldots$ & 1882.7 & 1.02 & 0.3 & 335 & \\
\hline \multirow[t]{2}{*}{ Si III] ............... } & 1892.0 & 11.80 & 3.9 & 800 & \\
\hline & 1892.0 & 17.45 & 5.7 & 3950 & \\
\hline$[\mathrm{Si} \mathrm{III}]+\mathrm{Si} \mathrm{III}] \ldots .$. & $\ldots$ & 30.27 & 9.9 & $\ldots$ & \\
\hline \multirow{2}{*}{$\mathrm{C}$ iII $]. . . \cdots \cdots \cdots$} & 1908.7 & 9.24 & 3.0 & $\dddot{880}$ & \\
\hline & 1908.7 & 42.61 & 14.0 & 5500 & \\
\hline \multirow[t]{2}{*}{$\mathrm{C}$ III] Sum ........... } & $\ldots$ & 51.85 & 17.0 & $\ldots$ & \\
\hline & & Model C & & & \\
\hline \multirow[t]{3}{*}{ Fe III UV34 .......... } & 1895.5 & 6.33 & 2.1 & $\ldots$ & 0.7 \\
\hline & 1914.1 & 8.98 & 3.0 & $\ldots$ & 1.0 \\
\hline & 1926.3 & 4.50 & 1.5 & $\ldots$ & 0.5 \\
\hline Fe III Sum ........... & $\ldots$ & 19.81 & 6.5 & $\ldots$ & $\ldots$ \\
\hline & 1882.7 & 1.02 & 0.3 & 335 & \\
\hline \multirow{2}{*}{ Si $\mathrm{mI}] \ldots \ldots \ldots \ldots \ldots \ldots$} & 1892.0 & 10.12 & 3.3 & 800 & \\
\hline & 1892.0 & 17.49 & 5.7 & 3950 & \\
\hline$[\mathrm{Si} \mathrm{III}]+\mathrm{Si} \mathrm{III}] \ldots \ldots$ & $\ldots$ & 28.63 & 9.3 & $\ldots$ & \\
\hline \multirow[t]{2}{*}{$\mathrm{C}$ III $] \ldots \ldots \ldots \ldots \ldots$} & 1908.7 & 9.24 & 3.0 & 880 & $\ldots$ \\
\hline & 1908.7 & 4.26 & 14.0 & 5500 & $\ldots$ \\
\hline $\mathrm{C}$ III] Sum ............ & $\ldots$ & 51.87 & 17.0 & $\ldots$ & \\
\hline
\end{tabular}

${ }^{a}$ These model measurements are also listed in Tables 3 and 4.

The broad component of C IV is relatively weak compared to the other UV lines (Table 3; Fig. 5c). Marziani et al. (1996) define a strong broad C IV component in the HST spectropolarimetry data ${ }^{23}$ of I $\mathrm{Zw} 1$, but they fitted the component to emission which we identify as Si II, possibly $\mathrm{Fe}$ II and blueshifted C IV. Given the lack of deblending or detailed line identification their broad component fit has an $\mathrm{EW}=21 \AA$, compared to ours of $\sim 9 \AA$.

\subsection{Is Iron Emission Associated with Outflows?}

Baldwin et al. (1996) study the nature of the kinematic components in the emission line spectra of seven quasars and, based on one of them, Q0207-398, propose that three different components are present in the line profile. They argue that Q0207-398 is a "misaligned" broad absorption line (BAL) quasar as the blueshifted line emission in Q0207-398 is typically absorbed in BAL quasar spectra. Baldwin et al. also connect this blueshifted emission and

\footnotetext{
${ }^{23}$ Note, their flux level is different from our spectrum and that of L97.
}

absorption to the expanded photospheres of (bloated) stars close to the central source and argue that $\mathrm{Al}$ III $\lambda 1857$ emission is an indicator of the presence of these stars.

The UV spectrum of Q0207-398 bears a remarkable resemblance to that of I $\mathrm{Zw} 1$ (narrow line cores, strong $\mathrm{Al}$ III, $\mathrm{Fe}$ II, and $\mathrm{Fe}$ III emission, high-density emitting regions, and blueshifted emission in the high-ionization lines), and the different kinematical components in Q0207-398 are similar to the emitting regions at different redshifts in I Zw 1 (Phillips 1976; van Groningen 1993), providing further support for the argument that I $\mathrm{Zw} 1$ is (also) a "misaligned" BAL quasar (L97). Baldwin et al. (1996) connect the $\mathrm{Al}$ III emission to outflows (and stars). So as the $\mathrm{Al}$ III and $\mathrm{Fe}$ III emission strengths appear to be connected (e.g., HB86) we speculate that the iron emission itself is somehow related to the presence of the outflows and/or to the stars. The fact that the iron line profiles do not appear asymmetric or are significantly blueshifted indicates that this emission does not originate in the outflowing gas itself. In any case, strong iron (Fe II and $\mathrm{Fe}$ III) emission may be 
connected with high densities (HB86; Joly 1991; Baldwin et al. 1996; Lawrence et al. 1997; Kuraszkiewicz et al. 2000).

\subsection{Is the Iron Spectrum of I $Z w 1$ Typical?}

An underlying assumption for using the I $\mathrm{Zw} 1$ spectrum as an iron template is that the iron emitting mechanism in this target is similar to that of typical quasars and that the spectrum provides a good representation of the iron transitions and iron line ratios observed in AGNs and quasars. We therefore address the question of how typical the emission lines, including iron, in I Zw 1 may be.

I $\mathrm{Zw} 1$ has a number of unusual properties in addition to strong $\mathrm{Fe}$ II emission. It has strong $\mathrm{Ca}$ II emission at $\lambda \lambda 8498$, 8542,8662 (the infrared triplet, permitted lines) and $\lambda \lambda 7291$, 7312 (forbidden lines) (Phillips 1976; van Groningen 1993; Persson \& McGregor 1985), strong C III $\star \lambda 1175$ emission (Laor et al. 1997a; L97), strong Si III] $\lambda 1892$, weak C III] $\lambda 1909$, strong IR emission (e.g., Rieke \& Low 1972; Rieke 1978) plus strong $\mathrm{Fe}$ III emission transitions in its spectrum (Figs. 1-3).

Strong Ca II emission is observed in $\sim 30 \%$ of all quasars and AGNs (Netzer 1990) and is generally thought to be emitted in the deepest interior of BLR clouds (Persson \& McGregor 1985; van Groningen 1993) with very high column densities $\left(N_{\mathrm{H}}>10^{24.5} \mathrm{~cm}^{-2}\right)$. Strong Ca II emitters, however, do not otherwise appear different from those of the average AGN population (Netzer 1990).

$\mathrm{C} \mathrm{III} \star \lambda 1175$ is not a commonly detected metastable transition of C III in AGNs and has previously most often been detected in absorption (e.g., Bromage et al. 1985; Kriss et al. 1992), perhaps due to the difficulty in identifying very broad, but weak features (§ 1.3). See, e.g., Laor et al. (1995) and Hamann et al. (1997) for weak and marginal detections in emission. The line may result from the enhanced density (see discussion by L97) of the emitting medium already deduced for I $\mathrm{Zw} 1$. Several studies show a trend toward strong $\mathrm{Fe}$ II, $\mathrm{Fe}$ III, and/or $\mathrm{Al}$ III at higher BLR densities (§5.4). If high densities are a common property of strong iron emitters, the iron spectrum is not expected to be unusual.

Strong Fe III features are more prevalent in I Zw 1 (Table 4) than in previous AGN studies, although $\mathrm{Fe}$ III features at 2070 and $\sim 2420 \AA$ have been reported in the past (HB86; L97). Our study suggests, however, this difference is not real but due to misidentifications in the past. Francis et al. (1991) note unidentified features in the 2000-2200 and 2900-3200 Å regions, consistent with $\mathrm{Fe}$ III lines according to our identifications (see references to Table 4). The presence of both ions is potentially important for deriving the physical conditions in the iron-emitting regions, because the details of the iron emission spectrum are highly sensitive to the physical conditions (e.g., Netzer 1980; D. Verner 1997, private communication).

Lipari et al. (1993) and Lipari (1994) link extremely strong optical Fe II emission to starburst activity. Though I Zw 1 is currently undergoing vigorous star formation (based on Barvainis et al. 1989; Eckart et al. 1994; Sanders \& Mirabel 1996), the iron emission observed in AGNs is not characteristic of emission from star forming regions. Pure starburst galaxies (e.g., NGC 7714) do not emit permitted Fe II emission, only IR forbidden lines (e.g., [Fe II] $1.6 \mu \mathrm{m}$; L. Ho 1998, private communication) mainly because the stellar ionizing continuum is too soft. To obtain the permitted lines the hard, nonthermal continuum from a central AGN source is needed to penetrate to the high-density interior of the BLR clouds (e.g., Netzer 1990).

Based on the above discussion, we find no strong evidence that the iron emission in I $\mathrm{Zw} 1$ is unusual compared with that of the general quasar population. This is confirmed by our successful fitting of the iron emission in several quasar spectra (§ 4.3).

\section{SUMMARY AND CONCLUSIONS}

We have presented a UV iron template based on HST archival data of $\mathrm{I} Z \mathrm{~W} 1$, and described the method with which the template was generated. Compared to previous empirical templates (Corbin \& Boroson 1996) this covers a large range (1250-3090 $⿱$ ) ), was generated by careful fitting of the non-iron emission and absorption lines, and allowed the generation of separate $\mathrm{Fe}$ II and $\mathrm{Fe}$ III templates. We have demonstrated its application to fit and remove the $\mathrm{Fe}$ II (and $\mathrm{Fe}$ III) emission in spectra of several quasars, including 3C 273, allowing subsequent studies of weak and heavily blended emission lines (e.g., C III]) free from the large uncertainties otherwise associated. This shows that the iron emission in $\mathrm{I} Z \mathrm{~W} 1$ is sufficiently similar to that in other broad-lined quasars to be useful. Although it has limitations, the iron template fitting process is an important tool to eliminate and study the iron emission in active galaxies, at least until accurate theoretical models are developed.

We confirm previous results reporting the presence of blueshifted emission (i.e., blue profile asymmetry) in the spectrum of I $\mathrm{Zw}$ 1, especially in the high-ionization lines (L97), and of several emission regions of different (absolute) redshift, line width and physical conditions (Phillips 1976; van Groningen 1993). The presence of these regions along with the UV spectral properties are consistent with the picture suggested by Baldwin et al. (1996) in which the blueshifted emission originates in the same region giving rise to the BAL troughs in BAL quasars. This may connect the NLS1's with BALs, perhaps through source orientation. We also argue that (strong) iron emission may be connected with high densities and to outflowing material.

We discuss the fact that the $\mathrm{Si}$ IV $+\mathrm{O}$ IV] $\lambda 1400$ feature is very strong relative to $\mathrm{C}$ IV. This can be explained by a simultaneous weakening of the Carbon lines and strengthening of the Silicon lines, common to narrow line Seyfert 1's (Kuraszkiewicz et al. 2000), probably due to a combination of high densities and a low-ionization parameter.

Based on earlier $I U E$ measurements we find $\mathrm{I} \mathrm{Zw} 1$ to have brightened a factor $\sim 2$ in its continuum emission since 1978-1982.

We are grateful to Adam Dobrzycki for help and guidance on the I $\mathrm{Zw} 1$ HST data processing, Luis Ho and Ari Laor for comments on early versions of the paper, and Kirk Korista, Ari Laor, Anil Pradhan, Dima Verner, and Beverly Wills for useful discussions. We also owe thanks to Bev Wills for kindly providing digital data of the 3C $273 \mathrm{UV}$ optical spectrum and the associated Fe II models presented by Wills et al. (1985). We are grateful to an anonymous referee for very helpful comments, leading to significant improvements of this paper. Many thanks are also due to the IRAF help desk, in particular Frank Valdes and Mike Fitzpatrick, for invaluable help with and guidance through IRAF problems. MV is very pleased to thank the Smithson- 
ian Astrophysical Observatory for their hospitality and gratefully acknowledges financial support from the Danish Natural Sciences Research Council (SNF-9300575), the Danish Research Academy (DFA-S930201), a Research Assistantship at Smithsonian Astrophysical Observatory
(NAGW-4266, NAGW-3134, NAG5-4089; P.I.: Belinda Wilkes), and the Columbus Fellowship at The Ohio State University. B. J. W. gratefully acknowledges financial support from NASA contract NAS 8-39073 (Chandra X-ray Center).

\section{APPENDIX A}

\section{COMMENTS ON INDIVIDUAL SPECTRAL FEATURES IN I ZWICKY 1}

Unless otherwise noted, the identifications of $\mathrm{Fe}$ II and $\mathrm{Fe}$ III emission features are made using the iron line lists available on the Web and from recent publications (Fuhr et al. 1988; Penston et al. 1983; Ekberg 1993; Giridhar \& Arellano Ferro 1995 ; Nahar 1995; Nahar \& Pradhan 1996; Quinet 1996; Quinet et al. 1996; Kurucz 1997). When (iron) residuals are said to be removed or excluded (i.e., subtracted) from the template, it is done in order to prevent an overestimation of the iron emission strength and subsequent overcorrection for the iron emission when the template is applied to quasar spectra. In addition, some line fits may require a local continuum level (different than the global continuum setting). However, the EW measurements are always with respect to the global continuum.

$\lambda \lambda 1120-1135$ emission. - Fe III UV1 emission is clearly detected but is heavily affected by absorption (Figs. 1 and 2). Due to the uncertainty in correcting for this absorption in the noisier end of the spectrum, we chose not to include the region $1075-1135 \AA$ in the template.

$\mathrm{C} \mathrm{III} \star \lambda 1176$ emission.- This emission line (Figs. 1, 2, and $5 a$ ) is an excited metastable level of $\mathrm{C}$ III and rarely detected in AGN spectra (but see Laor et al. 1995; Hamann et al. 1997, and see for absorption detections Kriss et al. 1992; Bromage et al. 1985). Its implications are discussed in $\S 5.5$, and Laor et al. (1997a, 1997b) also discuss possible excitation mechanisms of this transition.

$\lambda \lambda 1150-1245$ range. - Faint emission of Fe II is expected in this wavelength range (Figs. 1 and 2). The leftover residuals from subtracting the fits to the very strong, non-iron lines (especially Ly $\alpha$ ) are rather noisy and do not obviously match expected Fe II transitions. We therefore choose to remove these residuals from the template (Fig. 3).

Ly $\alpha \lambda 1216$ emission. - Two narrow components are necessary to reproduce the shape of the narrow line core (Fig. 5a). The weaker of the two components is blueshifted $\left(\sim 900 \mathrm{~km} \mathrm{~s}^{-1}\right)$ relative to the peak position of the stronger component. The high $\mathrm{S} / \mathrm{N}$ data constrain the fit well. Although the Ly $\alpha$ profile does not closely resemble that of the Balmer lines (cf. $\mathrm{H} \alpha$ and $\mathrm{H} \beta$ modeled by BG92, and L97), the profile shapes of all three are well reproduced by one broad and two narrow Gaussian components, one of which is blueshifted resulting in an asymmetric profile.

$\mathrm{O} v \lambda 1218$ emission?.-The feature clearly appears once the fit to Ly $\alpha$ is subtracted and the line position is well defined at $1218 \AA$ (Fig. 5a). The identification (Penston et al. 1983) is uncertain as one would expect a high blueshift of the line similar to the other high-ionization lines.

$\mathrm{N} v \lambda \lambda 1238,1243$ emission.-When the absorption is corrected, a blue asymmetric profile (Fig. $5 a$ ) is left, similar in appearance to the nondeblended profiles of Si IV + O IV] and C IV. The doublet profile is fitted well with three Gaussian components, one of which is considerably blueshifted $\left(\sim 2500 \mathrm{~km} \mathrm{~s}^{-1}\right)$ relative to the $\lambda 1243$ line. The remaining doublet feature is blueshifted $\sim 900 \mathrm{~km} \mathrm{~s}^{-1}$ similar to the blueshifted Ly $\alpha$ emission, relative to the rest frame.

$\lambda \lambda 1230,1234$ absorption.-L97 identify this absorption (Fig. $5 a)$ as due to $\left(\sim 2000 \mathrm{~km} \mathrm{~s}^{-1}\right)$ blueshifted $\mathrm{N} \mathrm{v}$ absorption intrinsic to I Zw 1, supported by their detection of weak associated absorption in Ly $\alpha$ and C IV also at this blueshift.

The absorption lines cannot be due to $\mathrm{O}_{\mathrm{I}} \lambda 1306$ and Si II $\lambda 1309$, although the wavelengths coincide very well with their laboratory wavelengths, as these transitions originate in slightly excited states which are not common in the ISM (Savage et al. 1993).

$\lambda \lambda 1257-1268$ emission. - This feature is identified as Si II $\lambda 1263$ (Fig. 5a). Due to the detection of Fe II UV9 emission at $\lambda \lambda 1270-1280$, Fe II (UV9 $\lambda \lambda 1250-1280 \AA$; Fig. 4) is also expected to be blended with this Si II multiplet. The wavelengths of the Gaussian components (Table 3) fitted to the Si II line blend are uncertain, due in part to the short wavelength emission being blended with the Ly $\alpha$ fit (and hence partly subtracted) and in part to blending with Fe II.

$\mathrm{Si}$ III $\star \lambda 1297$ emission?.-The identification of this feature is not confirmed (e.g., Fig. 2). It is not clear whether the emission is due to $\mathrm{Si}$ III ${ }^{\star}$ emission or to a blueshifted component of O I $\lambda 1306+\mathrm{Si}$ II $\lambda 1309$, similar to that found in the high-ionization lines. In the latter case the blueshift is $\sim 2000 \mathrm{~km} \mathrm{~s}^{-1}$, in agreement with the blueshift of the high-ionization lines. A third possibility is faint $\mathrm{Fe}$ III $\lambda 1298.6$ and Fe II $\lambda \lambda 1297,1299$ emission which is expected based on the iron line lists, although the observed emission feature appears too strong.

O I 21304 emission.-Faint Fe II appears, especially in the blue wing, but the iron emission could not be deblended (Fig. 2).

$\lambda 1343$ feature.-The identification is uncertain (Figs. 1 and 2). No obvious Fe II, Fe III or other commonly observed quasar broad emission line matches the wavelength position. Candidate identifications are $\mathrm{O}$ IV, $\mathrm{Ca}$ II, $\mathrm{Fe}$ III, and Fe v. A coincidence occurs with some Fe III transitions, as noted in Table 3, but it is not clear whether an isolated feature, as observed, is expected. $\mathrm{Ca}$ II is also observed in the IR ( $(5.5)$, but this $\lambda 1343$ feature is redshifted with respect to the expected Ca II position (Table 3).

$\mathrm{Si}$ IV $+\mathrm{O}$ IV] $\lambda 1400$ emission.-Faint Fe II emission is expected at $\lambda 1393$ and $\lambda 1408$. A faint Fe III feature is expected at $\lambda 1395$, which is identified in the residuals after subtracting the $\mathrm{Si}$ IV + O IV] fits. The strength of Fe III $\lambda 1395$ is likely to be underestimated: the feature is heavily blended with the Si IV $+\mathrm{O}$ IV] emission and the data do not permit sufficient constraints to be placed on each contributing emission component. 
Blueshifted emission is clearly present in this line complex (Fig. 5b). A deblending of each of the components is not straight forward due to the severe blending of the components emitted in the rest frame with the blueshifted line emission. Hence, each individual component has a larger uncertainty than the complex fit as a whole. We interpret the complex as follows: the "rest frame" emission has a general blueshift of $\sim 900 \mathrm{~km} \mathrm{~s}^{-1}$ relative to the $\mathrm{Mg}$ II line peak (consistent with Ly $\alpha, \mathrm{N} v, \mathrm{C}$ IV, and Al III) and the excess blueshifted emission has a projected velocity of $\sim-1100 \mathrm{~km} \mathrm{~s}^{-1}$ relative to the former ("rest frame") emission and a total blueshifted velocity of $\sim 2000 \mathrm{~km} \mathrm{~s}^{-1}$ relative to the true rest frame. The complex clearly has a broad component, which displays the strongest blueshift of $2000 \mathrm{~km} \mathrm{~s}^{-1}$ (Table 3).

The line complex as a whole is strong relative to C IV, most likely because C IV is weak and/or the density is high and the ionization parameter is low (see discussion in $\S 5.3$ ). Alternatively, the iron emission may be stronger than we currently suspect, making the Si IV + O IV] emission appear stronger and contributing to the high $\lambda 1400 / C$ IV line ratio.

$\mathrm{N}$ IV] $\lambda 1486$ emission. - This emission line is not detected (Figs. 2 and 5c). It may, however, be weak and heavily blended with iron emission.

$\lambda \lambda 1515,1517$ dips.-These features (Fig. 5c) appear very similar to some of the Galactic absorption doublets, but comparison with the low-dispersion IUE spectrum (Lanzetta et al. 1993) clearly show that this dip is a lack of emission surrounded by line emission, which we identify as Fe III (Table 4).

Si II $\lambda \lambda 1527,1533$ emission.-This doublet transition is almost certainly present (Fig. $5 c$ ). The lines are, however, strongly blended with C IV and possibly iron emission, and the individual line profiles and their strengths cannot be determined with confidence. Due to the apparent weakness of the broad C IV component we suspect the contribution from the Si II doublet to be significant. An alternative interpretation is that $C$ IV has a strong and rather blueshifted component of $\sim 6000 \mathrm{~km} \mathrm{~s}^{-1}$ relative to the rest frame. In that case, there must be several blueshifted emitting regions as the observed emission does not appear to reflect the doublet flux ratio which is estimated for the narrow C IV components (Table 3 and Fig. $5 c$ ). Fe III UV84 and weaker Fe III features are also expected to be present (see below).

C IV $\lambda 21548,1550$ emission.-Blueshifted emission appears present (Fig. 5c) but, due to the heavy blending, a wellconstrained Gaussian component fit (deblending) to determine the blueshift and flux is not possible. The data only support a weak broad component (Fig. 5c; Table 3). Narrow profiles were fitted to the peaks of the Si II $\lambda \lambda 1527,1533$ and C IV $\lambda \lambda 1548,1550$ doublet lines which are clearly resolved. The best fit to the $C$ IV narrow emission has slightly different widths of the transitions (Table 3). If the line width is fixed and a flux ratio of 2:1 is approximated a Gaussian residual appear (see the alternative fit to the narrow C IV emission in Table 3). In addition, a two-component feature at 1536 and $1539 \AA$, coinciding with Fe III UV84 emission (see below and Fig. 4), is required to fit the complex well. An alternative fit has two narrow components with a flux ratio of approximately 2:1 (Table 4) suggesting an additional blueshifted $\left(\sim 2300 \mathrm{~km} \mathrm{~s}^{-1}\right)$ component of $\mathrm{C}$ IV emission. The likelihood that this emission is $\mathrm{Si}$ i is probably rather low as expected strong transitions at $\sim 1542,1547$, 1553, and 1550-1600 ̊ (Morton 1991) are not seen.

Fe III $\lambda \lambda 1520-1540$ emission. - The UV84 multiplet of Fe III among other Fe III lines of detectable strengths (1515.5, 1516.2, 1518.8, 1524.5, 1525.0, 1526.0, and 1527.0 А; see Table 4 and Fig. 4). are expected close to the positions of Si II and C IV; the most probable lines are expected at 1526, 1527, 1531-1532, 1538-1539, and 1550-1551 $\AA$ (Nahar \& Pradhan 1996). If these transitions are present $\mathrm{Si}$ II and C IV may be even weaker relative to the other resonance UV lines. The IUE spectrum presented by Lanzetta et al. (1993) does not show any signs of strong Fe III emission in this region. But since I Zw 1 has brightened by a factor of $\sim 2(\S 2)$ in continuum flux and both the line strengths and the continuum slope have changed since the IUE data were taken, this is not a strong argument against the presence of Fe III in the HST spectrum. Due to the uncertainty in the identification and strength of this possible iron emission feature, it is not included in the $\mathrm{Fe}$ III and $\mathrm{Fe}$ II templates.

$\lambda \lambda 1807-1875$ emission.-Fe III (UV97,117 at $1830-1855 \AA$ and UV53,63 at $1850-1871 \AA$; see Fig. 4) is the most likely identification, but fainter Fe II (UV65,66) emission is also consistent with some of the residuals in the range 1807-1875 (Fig. $5 d$ ). Fe II is, however, expected to dominate in the range 1807-1836 $\AA$ (most of the weaker, blended iron multiplets are not marked in Fig. 4). Due to the heavy blending, no attempt were made to separate the individual $\mathrm{Fe}$ II and Fe III contributions. A compromise was made: the emission at $1837-1872 \AA$ is treated entirely as Fe III emission (and included in the Fe III emission model and Fe III template) while the $1802-1836 \AA$ wavelength range is adopted as pure Fe II. This is clearly an approximation.

Al III $\lambda \lambda 1854,1863$ emission. - The doublet is relatively strong, and well resolved in this spectrum (Fig. $5 d$ ). A broad base component is clearly present along with a number of iron emission transitions. We detect no obvious excess blueshifted emission and find that the most likely identification of the non-Al III emission in the 1850-1875 $\mathrm{\AA}$ region is that of iron (Figs. 4 and $5 d$ ).

Si III] $\lambda 1883$ emission.-This was identified by L97 as possible Si III] emission (Fig. $5 d$ ). The feature is observed at $1880.3 \AA$. The Fe II UV126 multiplet is also expected (and consistent with the emission) at 1864.6, 1864.7, and 1880.97 $\AA$, providing an alternative identification. The $1864 \AA$ lines are blended severely with the Al III emission and the identification cannot be confirmed via line strength arguments. When in doubt we prefer to underestimate the iron emission (see the introduction to this section and § 3.3.2), so we do not include this feature in the template.

Fe III $\lambda 21892-1906$ emission.- The residuals between 1898 and $1907 \AA$ were not fitted with Gaussian components (Fig. 5d). However, they were isolated by subtraction of fitted non-iron lines and then included in the Fe III template (Fig. 3). With the Fe III UV34 $\lambda 1914$ emission feature observed at $\sim 1912 \AA$, the $1895 \AA$ transition is expected at $1893 \AA$, the position of a weak feature (Fig. 4). Given the apparent weakness of the feature at $1893 \AA$ the Fe III UV34 multiplet is not likely to dominate the strength of Si III] $\lambda 1892$, detected at $\sim 1890 \AA$ (Fig. $5 e$ ). The UV34 triplet $(1895 \AA, 1914 \AA$, $1926 \AA$ ) may have optically thin line ratios 0.9:1.0:0.3 (§5.2) while all transitions have equal strengths in the optically thick limit. As discussed in $\S 5.2$, our modeling of the Fe III UV34 triplet show that no tight constraints can be placed on the relative line ratios. However, this modeling does indicate an optically thin ratio (cf. models B and C in Fig. 5e). The spectrum indicates that this triplet is emitted 
in the optically thin region given the apparent relative weakness of the 1895 and $1926 \AA$ features. The relatively strong Si III]/ $\mathrm{C}$ III] line ratio combined with the relatively strong Fe III emission indicates rather high BLR densities.

C III] $\lambda 1909$ emission complex.-The emission feature is a complex blend of emission lines, but the spectral resolution and the narrow widths of the line cores permit a separation of the narrow-line cores of the $\mathrm{Si}$ III] $\lambda 1892, \mathrm{C}$ III] $\lambda 1909$, and $\mathrm{Fe}$ III $\lambda \lambda 1914,1926$ lines (Fig. 5d). Two broad components of Si III] and C III] could be fitted (Table 3), although their individual fits, including their strengths, are not well constrained. A number of other Fe III transitions are detected in and around this line complex (Fig. 4 and Table 4).

Fe III $\lambda 1914$ emission.- The emission at 1907-1918 $\AA$ is consistent with contributions from a number of intermediate to strong Fe III multiplets; among them, the strongest are UV34, 101, and 83 and the weaker ones are UV57, 135, and 108. This heavy blending is probably responsible for the residual UV34 ( $\lambda 1914)$ feature appearing stronger than, e.g., UV68 $\lambda 1952$, and UV50 $\lambda \lambda 1987-1996$ (Fig. 4). If UV51 ( $\lambda \lambda 1915.1,1922.8,1930.4,1937.3,1943.4)$ is present, it must be faint. See $\S 5.2$ for discussion of the relative Fe III UV34 contribution.

$\mathrm{N} \mathrm{II]} 22141$ emission.-The pseudo-continuum was used as the local continuum level (Fig. 2).

$\lambda \lambda 2160-2190$ region. - The absolute strengths of the emission and absorption lines are not straightforward to determine in the $\sim 2100-2250 \AA$ A region due to the presence of dust absorption features $(\S 2)$ and the uncertainty in the placement of the absolute continuum level (Fig. 2). According to the line lists of Nahar (1995), the expected Fe II transitions are faint between 2100 and $2200 \AA$.

$\lambda \lambda 2250-2290$ region.-The possible Fe III UV73, 153 ( $\lambda \lambda 22258.1,2274.7,2277.6,2285.7)$ emission is not fitted and not included in the Fe III emission model as the identification is uncertain, due to poor wavelength coincidence.

Fe III UV47 $\lambda 22418,2438$ emission.- The profile was fitted with three Gaussian components, one of which accounts for a broad component and the weaker of the two narrow components is blueshifted $\sim 500 \mathrm{~km} \mathrm{~s}^{-1}$ with respect to the stronger one (Fig. 5f). The choice of including a broad component was based on the appearance of the H $\alpha$ profile (Phillips 1977; L97).

Unfortunately, the spectrum does not offer good constraints on the absolute strengths of the broad Fe III UV47 component fit. A good fit requires that the actual continuum level for the fitting be placed $\sim 6 \%$ below the observed flux level at the apparent line base.

The profile is also consistent with a fit of two narrow Gaussian components (and no broad component) and the actual continuum level (for the fitting) is placed at the observed flux level, but with the known similarity between the (Balmer) hydrogen lines and the Fe II lines, the presence of a broad Fe III component is much more likely (see also L97).

$\lambda 2435$ emission feature.- The identification is uncertain. Possibilities include the (slightly blueshifted) O II (UV18) $\lambda \lambda 2433.6$, 2444.4, 2445.6. Its blueshift $\left(\sim 775 \mathrm{~km} \mathrm{~s}^{-1}\right)$ is consistent with that of [O II] $\lambda 2470$. Some Si I transitions are also expected nearby (Si I UV45 and UV2; Table 3). The feature was fitted with the pseudo-continuum as the local continuum. The best fit was obtained with two Gaussians (Fig. $5 f$ ); it is not clear how the individual Gaussian parameters (width and position) relate to those of the multiplet components.

[O II] 22470 emission.- - None of the lists of Fe II and Fe III transitions (including that by Giridhar \& Arellano Ferro 1995) predict strong transitions at this wavelength. L97 identify the feature as [O II] (Figs. 2 and $5 f$ ). The pseudo-continuum acts as the local continuum level in the component fitting.

$\lambda 2481$ emission feature.- The identification is uncertain. No obvious strong iron emission is expected at this position. C I $\lambda 2478$ is a possible identification. The fit is based on the pseudo-continuum (Fig. $5 f$ ).

Mg II $\lambda \lambda 2795,2803$ emission.- The systemic redshift was measured with a two-Gaussian component fit (one doublet; § 3.3.2) to the narrow line core early in the course of this work. A more detailed and improved line fit reveals a second doublet with slightly larger line widths blueshifted $400 \mathrm{~km} \mathrm{~s}^{-1}$ relative to the stronger doublet at rest in the quasar frame (Fig. $5 g$ and Table $3)$. Each doublet has a thermalized line ratio $(=1: 1)$.

The fit displays evidence for excess blueshifted emission similar to that in Ly $\alpha, \mathrm{N} v, \mathrm{He}$ II and possibly Si IV + O IV], and C Iv. L97 fitted the entire doublet (broad and narrow components) using $\mathrm{H} \alpha$ as a template profile. Two such profiles with relative strengths 1.2:1 fitted the line well, and they argued for higher electron densities in the $\mathrm{Mg}$ II line gas based on the thermalized doublet ratio $(\sim 1: 1)$.

It is clear from Figure $5 g$ that no iron emission is left in the $2770-2820 \AA$ range after subtraction of the $\mathrm{Mg}$ II fit. Fe II emission is not expected to be strong at the position of Mg II (D. Verner 1997, private communication) but is not entirely absent either (see Fig. 4 and Verner et al. 1999). As the data cannot constrain the strength of the broad component well, we prefer to underestimate the Fe II strength to prevent an overcorrection of the iron emission when the iron template is applied (see §3.3.2). This choice of the local underlying continuum is consistent with that found by L97.

\section{APPENDIX B}

\section{THE 3C 273 HST DATA}

In $\S 4.3$ HST archival data, covering the full UV range from Ly $\alpha$ to beyond $\mathrm{Mg}$ II, of the nearby radio-loud quasar, 3C 273, were used to demonstrate the applicability of the I Zw 1 based Fe II and Fe III templates to the iron emission in other AGNs. The 3C 273 UV spectrum was generated by combining data from the FOS and the Space Telescope Imaging Spectrograph (STIS). We used the 1" aperture G130H, G190H, and G270H FOS grating spectra from 1991 January 16, 14, and 15, respectively, which only partially cover the Fe II bump around $\mathrm{Mg}$ II. The spectral range was extended longward of $\mathrm{Mg}$ II by combining these spectra with a $52 \times 2$ aperture G430L STIS grating spectrum observed on 1999 January 31 . The latter 
spectrum was multiplied by a factor 1.142 to coincide with the average flux level of the FOS spectra. The G270H spectrum was truncated at $3200 \AA$ (observed) before being combined with the G430L spectrum, which then provided the Mg II line profile. There is a $\sim 50 \AA$ gap between the $\mathrm{G} 130 \mathrm{H}$ and $\mathrm{G} 190 \mathrm{H}$ spectra. An interpolation was performed using the average continuum flux levels in the $\sim 40 \AA$ ends of each spectrum, avoiding absorption lines, to facilitate the fitting of the iron emission. The spectra were resampled to a common dispersion of $2.37 \AA$ pixel $^{-1}$ in the rest frame based on the G430L spectrum. The lower resolution of the G430L spectrum did not affect the iron fitting in any way. This was confirmed by a simultaneous fitting to the higher resolution spectrum made from the FOS spectra only $\left(0.44 \AA\right.$ pixel $^{-1}$, rest frame; no fitting to the full "small iron bump" was possible, however) which showed no significant differences in the fitted models. This is most likely due to the relative broadness $\left(\sim 4000 \mathrm{~km} \mathrm{~s}^{-1}\right)$ of the lines in the spectrum $(\S 4.3)$.

Baldwin, J. A. 1977, ApJ, 214, 679

Baldwin, J. A., Ferland, G., Korista, K., \& Verner, D. 1995, ApJ, 455, L119

Baldwin, J. A., et al. 1996, ApJ, 461, 664

Barlow, T. A., \& Sargent, W. L. W. 1997, AJ, 113, 136

Barvainis, R., Alloin, D., \& Antonucci, R. 1989, ApJ, 337, L69

Bergeron, J., \& Kunth, D. 1984, MNRAS, 207, 263

Boggess, A., et al. 1978, Nature, 275, 372

Boller, Th. Brandt, W. N., \& Fink, H. 1996, A\&A, 305, 53

Boroson, T. A., Persson, S. E., \& Oke, J. B. 1985, ApJ, 293, 120

Boroson, T. A., \& Green, R. F. 1992, ApJS, 80, 109 (BG92)

Bromage, G. E., et al. 1985, MNRAS, 215, 1

Brotherton, M. S., Tran, H. D., Van Breugel, W., Dey, A., \& Antonucci, R. R. J. 1997, ApJ, 487, L113

Cardelli, J. A., Clayton, G. C., \& Mathis, J. S. 1989, ApJ, 345, 245

Carson, J. E., et al. 2000, BAAS, 196, 5015

Condon, J. J., Hutchings, J. B., \& Gower, A. C. 1985, AJ, 90, 1642

Collin-Souffrin, S., Dumont, S., Joly, M., \& Péquignot, D. 1986, A\&A, 166, 27

Collin-Souffrin, S., Hameury, J.-M., \& Joly, M. 1988, A\&A, 205, 19

Corbin, M. R. 1997, ApJS, 113, 245

Corbin, M., \& Boroson, T. A. 1996, ApJS, 107, 69

Courvoisier, T. J.-L., \& Paltani, S. 1992, IUE-ULDA, Access Guide No. 4A

\& 4B (ESA-SP 1153A, 1153B; Noordwijk: ESA)

Davidson, K., \& Netzer, H. 1979, Rev. Mod. Phys., 51, 715

Dumont, A. M., \& Collin-Souffrin, S. 1990, A\&A, 229, 313

Eckart, A., van der Werf, P. P., Hofmann, R., \& Harris, A. I. 1994, ApJ, 424, 627

Ekberg, J. O. 1993, A\&AS, 101, 1

Elvis, M., Lockman, F. J., \& Wilkes, B. J. 1989, AJ, 97, 777

Elvis, M., et al. 1994, ApJS, 95, 1

Ferland, G. J., \& Persson, S. E. 1989, ApJ, 347, 656

Forster, K., Green, P. J., Aldcroft, T. L., Vestergaard, M., Foltz, C. B., \&

Hewett, P. C. 2001, ApJ, in press

Francis, P. J., Hewett, P. C., Foltz, C. B., Chaffee, F. H., Weyman, R. J., \& Morris, S. L. 1991, ApJ, 373, 465

Fuhr, J. R., Martin, G. A., \& Wiese, W. L. 1988, J. Phys. Chem. Ref. Data, 17 , suppl. 4

Gaskell, C. M. 2000, NewA Rev., 44, 563

Giannuzzo, M. E., \& Stirpe, M. G. 1996, A\&A, 314, 419

Giridhar, S., \& Arellano Ferro, A. 1995, Rev. Mexicana Astron. Astrofis., 31,23

Greenstein, J. L., \& Schmidt, M. 1964, ApJ, 140, 1

Halpern, J. P., \& Oke, J. B. 1987, ApJ, 312, 91

Hamann, F., Shields, J. C., Cohen, R. D., Junkkarinen, V. T., \& Burbidge, E. M. 1997, in ASP Conf. Ser. 113, Emission Lines in Active Galaxies: New Methods and Techniques, ed. B. M. Peterson, F.-Z. Cheng, \& A. S. Wilson (San Francisco: ASP), 96

Hartig, G. F., \& Baldwin, J. A. 1986, ApJ, 302, 64 (HB86)

Hummer, D. G., Berrington, K. A., Eissner, W., Pradhan, A. K., Saraph, H. E., \& Tully, J. A. 1993, A\&A, 279, 298

Johansson, S., \& Jordan, C. 1984, MNRAS, 210, 239

Joly, M. 1991, A\&A, 242, 49

Korista, K., Baldwin, J., Ferland, G., \& Verner, D. 1997, ApJS, 108, 401

Kraemer, S. B., \& Crenshaw, D. M. 2000, ApJ, 532, 256

Kriss, G. A., et al. 1992, ApJ, 392, 485

Kriss, G. A., Peterson, B. M., Crenshaw, D. M., \& Zheng, W. 2000, ApJ, 535,58

Krolik, J. H., \& Kallman, T. R. 1988, ApJ, 324, 714

Kuraszkiewicz, J., Wilkes, B. J., Czerny, B., \& Mathur, S. 2000, ApJ, 542, 692

Kurucz, M. 1997, CD-ROM 23 (Cambridge: SAO)

Kwan, J., \& Krolik, J. H. 1981, ApJ, 250, 478

Lanzetta, K. M., Turnshek, D., \& Sandoval, J. 1993, ApJS, 84, 109

Laor, A., Bahcall, J. N., Jannuzi, B. T., Schneider, D. P., \& Green, R. F. 1995, ApJS, 99, 1

Laor, A., Bahcall, J. N., Jannuzi, B. T., Schneider, D. P., Green, R. F., \& Hartig, G. F. 1994, ApJ, 420, 110

Laor, A., Jannuzi, B. T., Green, R. F., \& Boroson, T. A. 1997a, in ASP Conf. Ser. 113, Emission Lines in Active Galaxies: New Methods and Techniques, ed. B. M. Peterson, F.-Z. Cheng, \& A. S. Wilson (San Francisco: ASP), 116

Laor, A., Jannuzi, B. T., Green, R. F., \& Boroson, T. A. 1997b, ApJ, 489, 656 (L97)
Larkin, J. E. et al 2000, ApJ, 533,61

Lawrence, A., Elvis, M., Wilkes, B. J., McHardy, I., \& Brandt, W. N. 1997, MNRAS, 285, 879

Leighly, K. M. 1999, ApJS, 125, 317

Leitherer, C. 1995, HST Data Handbook, Version 2.0 (Baltimore: STScI)

Lipari, S. 1994, ApJ, 436, 102

Lipari, S., Terlevich, R., \& Macchetto, F. 1993, ApJ, 406, 451

Lockman, F. J. Jahoda, K. \& McCammon, D. 1986, ApJ, 302, 432

Marziani, P., Sulentic, J. W., Dultzin-Hacyan, D., Calvani, M., \& Moles, M. 1996, ApJS, 104, 37

Miller, H. R., Ferrara, E. C., McFarland, J. P., Wilson, J. W., Daya, A. B., \& Fried, R. E. 2000, NewA Rev., 44, 539

Moore, C. E. 1950, An Ultraviolet Multiplet Table, NBS Circ. 488, Section 1 (Washington: Dept. Commerce)

Morton, D. C. 1991, ApJS, 77, 119

Nahar, S. N. 1995, A\&A, 293, 967

Nahar, S. N., Bautista, M. A., \& Pradhan, A. K. 1997, ApJ, 479, 497

Nahar, S. N., Delahaye, F., Pradhan, A. K., \& Zeippen, C. J. 2000, A\&AS, 144,141

Nahar, S. N., \& Pradhan, A. K. 1996, A\&AS, 119, 509

Netzer, H. 1980, ApJ, 236, 406

1990, in Saas-Fee Advanced Course 20, Active Galactic Nuclei, ed.

T. J.-L. Courvoisier \& M. Mayor (Heidelberg: Springer), 57

Netzer, H., \& Wills, B. J. 1983, ApJ, 275, 445

Oke, J. B., \& Lauer, T. R. 1979, ApJ, 230, 360

Oke, J. B., Shields, G. A., \& Korycansky, D. G. 1984, ApJ, 277, 64

Osterbrock, D. E. 1989, Astrophysics of Gaseous Nebulae and Active Galactic Nuclei (Mill Valley: University Science Books)

Osterbrock, D. E., \& Pogge, R. 1985, ApJ, 297, 166

Paltani, S., \& Courvoisier, T. J.-L. 1994, A\&A, 291, 74

Penston, M. V. 1980, IUE-ESA Newslett., 5, 33

.1987, MNRAS, 229, 1P

Penston, M. V. et al. 1983, MNRAS, 202, 833

Persson, S. E., \& McGregor, P. J. 1985, ApJ, 290, 125

Peterson, B. M. 1997, An Introduction to Active Galactic Nuclei (New

York: Cambridge Univ. Press)

Peterson, B. M., Foltz, C. B., \& Byard, P. L. 1981, ApJ, 251, 4

Phillips, M. M. 1976, ApJ, 208, 37 1977, ApJ, 215, 746 1978, ApJ, 226, 736

Pian, E., \& Treves, A. 1993, ApJ, 416, 130

Pogge, R. W. 2000, NewA Rev., 44, 381

Quinet, P. 1996, A\&AS, 116, 573

Quinet, P., Le Dourneuf, M., \& Zeippen, C. J. 1996, A\&AS, 120, 361

Rees, M. J., Netzer, H., \& Ferland, G. J. 1989, ApJ, 347, 640

Rieke, G. H. 1978, ApJ, 226, 550

Rieke, G. H., \& Low, F. J. 1972, ApJ, 176, L95

Rodríguez-Ardila, A., Pastoriza, M. G., \& Donzelli, C. J. 2000, ApJS, 126, 63

Rodríguez-Pascual, P. M., Mas-Hesse, J. M., \& Santos-Lleo, M. 1997, A\&A, 327, 72

Rudy, R. J., Mazuk, S., Puetter, R. C., \& Hamann, F. 2000, ApJ, 539, 166

Sanders, D. B., \& Mirabel, I. F. 1996, ARA\&A, 34, 749

Sargent, W. L. W. 1968, ApJ, 152, L31

Savage, B. D., et al. 1993, ApJ, 413, 116

Schmidt, M., \& Green, R. F. 1983, ApJ, 269, 352

Seaton, M. J., Yan, Y., Mihalas, D., \& Pradhan, A. K. 1994, MNRAS, 266, 805

Sigut, T. A. A., \& Pradhan, A. 1998, ApJ, 499, L139

Smith, P. S., Schmidt, G. D., Allen, R. G., \& Hines, D. C. 1997, ApJ, 488, 202

Stark, A. A., et al. 1992, ApJS, 79, 77

Steidel, C. C., \& Sargent, W. L. W. 1991, ApJ, 382, 433

Tran, H. D., Cohen, M. H., \& Goodrich, R. W. 1995, AJ, 110, 2597

Ulvestad, J. S., Antonucci, R. J., \& Goodrich, R. W. 1995, AJ, 109, 81

van Groningen, E. 1993, A\&A, 272, 25

Verner, D. A., Barthel, P. D., \& Tytler, D. 1994, A\&AS, 108, 287

Verner, E. M., Verner, D. A., Korista, K. T., Ferguson, J. W., Hamann, F., \& Ferland, G. J. 1999, ApJS, 120, 101

Véron-Cetty, M.-P., \& Véron, P. 1993, A Catalogue of Quasars and Active Nuclei (6th ed.; ESO Sci. Rep. 13; Garching: ESO)

Wampler, E. J., \& Oke, J. B. 1967, ApJ, 148, 695

Wang, T.-G., Zhou, Y.-Y., \& Gao, A.-S. 1996, ApJ, 457, 111 
Weedman, D. W. 1986, Quasar Astronomy (Cambridge: Cambridge Univ. Press)

Wilkes, B. J. 1984, MNRAS, 207, 73 1986, MNRAS, 218, 331

2000, in Astrophysical Quantities, ed. A. N. Cox (4th ed.; New York: AIP), 585

Wills, B. J., \& Browne, I. W. A. 1986, ApJ, 302, 56

Wills, B. J., Netzer, H., \& Wills, D. 1980, ApJ, 242, L1 1985, ApJ, 288, 94
Wills, B. J., et al. 1995, ApJ, 447, 139

Wu, C.-C., Boggess, A., \& Gull, T. R. 1983, ApJ, 266, 28

Zheng, W., \& Malkan, M. A. 1993, ApJ, 415, 517

Zheng, W., Kriss, G. A., \& Davidsen, A. F. 1996, BAAS, 189, 1406 (98.13)

Zheng, W., Kriss, G. A., Davidsen, A. F., \& Kruk, J. W. 1995, ApJ, 454, L11

Zheng, W., Kriss, G. A., Telfer, R. C., Grimes, J. P., \& Davidsen, A. F. 1997, ApJ, 475, 469

Zwicky, F. 1971, Catalogue of Selected Compact Galaxies and of PostEruptive Galaxies (Guemligen: Zwicky) 